\title{
Gait Feature Extraction from Inertial Body Sensor Networks for Medical Applications
}

\author{
A Dissertation \\ Presented to \\ the Faculty of the School of Engineering and Applied Science \\ University of Virginia
}

in Partial Fulfillment

of the requirements for the degree

Doctor of Philosophy

By

Shanshan Chen

December

2013 
APPROVAL SHEET

The dissertation

is submitted in partial fulfillment of the requirements

for the degree of

Doctor of Philosophy

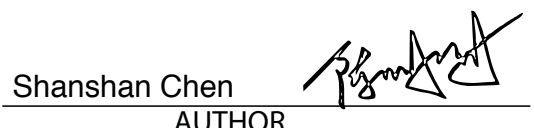

The dissertation has been read and approved by the examining committee:

\begin{tabular}{c} 
John Lach \\
\hline Advisor \\
Maité Brandt-Pearce \\
\hline Bradford Bennett \\
\hline Zongli Lin \\
Donald Brown
\end{tabular}

Accepted for the School of Engineering and Applied Science:

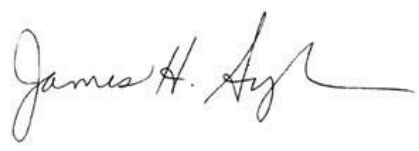

Dean, School of Engineering and Applied Science

December 
(C) Copyright

All rights reserved

by Shanshan Chen

December 2013 


\section{Abstract}

The emerging trend of Body Sensor Networks (BSNs) has excited research for enabling continuous and quantitative monitoring of human physiology, kinesiology, psychology, neuropsychology etc. In particular, one important type of BSN -- inertial BSNs -- has shown promising opportunities of monitoring human gait for various medical applications. These applications include: studying and monitoring gait pathology or degradation, assessing the efficacy of orthoses or prosthetics, and evaluating medical intervention for a gait-manifested neuropathy by comparing gait difference before/after the intervention. For such medical applications, inertial BSNs could not only function as a convenient and economical tool for quantitative medical observation, but also enable out-of-lab and long-term monitoring assessment, providing additional rich information for medical research and challenging clinical decisions beyond traditional, in-lab and/or qualitative clinical observation and further prompting medical research.

However, to demonstrate the feasibility of this technology, retrieving accurate and useful information (i.e., gait features) from inertial BSNs is essential and requires a combination of biomechanical knowledge and data processing innovation. Extracting spatial information (the basis of many gait features from traditional camera-based motion capture) from inertial BSN is particularly challenging due to sensor noise and integration 
drift, and existing gait feature extraction methods tend to target small subset of features for specific applications and deployment scenarios.

This work develops a systematic approach for gait feature extraction from inertial BSNs for medical applications. Techniques to tackle the challenge of tracking accurate spatial information in order to provide accurate kinematic information are introduced. Given the accurate kinematic information, linear analysis of extracting time domain gait features is applied. This work then explores the opportunity of leveraging biomechanics and applying nonlinear analysis in order to refine signal processing and enhance data separability and interpretability from extracted features. Also, machine learning approaches are explored for automating the process of feature selection and systematic error correction. Lastly, case studies for applying these techniques to various medical applications are discussed to exemplify the systematic approach. Overall, this systematic approach is aimed to conquer dominant challenges residing in inertial BSNs by extracting accurate gait features, transforming the extracted features into valuable medical knowledge, and enabling medical research requiring longitudinal gait analysis. 


\section{Acknowledgements}

My gratitude must first go to the best advisor in the world, Dr. John Lach. To me, he is not only a knowledgeable and insightful professor, but also a patient, supportive and inspiring mentor. When I encountered a challenging problem, his faith in me encouraged me to finish the last mile. I imagine years after my graduation, his admonitions about paying meticulous attention to detail in research and publishing, his excitement when a new idea is born after brainstorming, his humor in keeping up team morale when progress is frustrating, and his encouragement for me to push boundaries for the next challenge, will no doubt emerge in my future work.

I would also like to thank my PhD advisory committee: Dr. Brandt-Pearce, Dr. Bennett, Dr. Lin and Dr. Brown, who are renowned experts in their own fields and have provided me tremendous amount of suggestions and help towards my work. As this work is an interdisciplinary research in nature, their guidance is pivotal to my success.

My sharp-minded INERTIA teammates, who are fond of playing devil's advocates in our research discussion, taught me how to better articulate and present my ideas and examine my work with critical thinking. My special thanks would go to Dr. Harry Powell, Dr. Adam Barth, Jeff Brantley, Taeyoung Kim, Samuel Ridenour, Philip Asare, Juliana Su 
and Italo Armenti, for their generous support and brilliant suggestions for my research over the years.

I have also had happy and fulfilled five years at the University of Virginia. The prevailing spirit of learning and seeking for truth inside this campus has truly inspired me. I feel collaboration can almost happen every minute in every corner of the campus. Specially, I would like to thank all the medical collaborators I have closely worked with over the past few years: Dr. Jeffery Barth, Dr. Donna Broshek, Dr. Jason Freeman, and Dr. Myla Goldman.

My parents, although from time to time doubting whether their daughter should still study in universities after many years, have never hesitated to support me to adventure on my journey of pursuit of knowledge. Without their support a hemisphere away, I would not be able to come as far as today.

Finally, this research was largely supported by the National Science Foundation under grants IIS-1065262, CNS-1035771, CBET-0756645, and CBET-1034071. 


\section{Table of Contents}

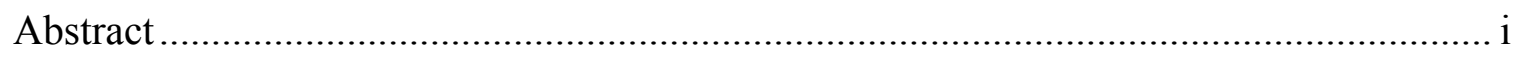

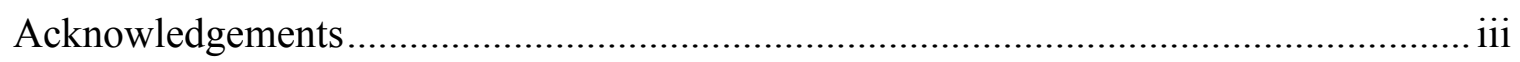

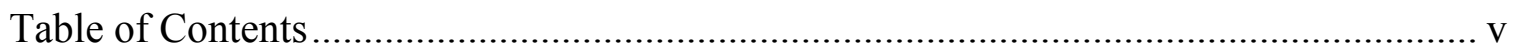

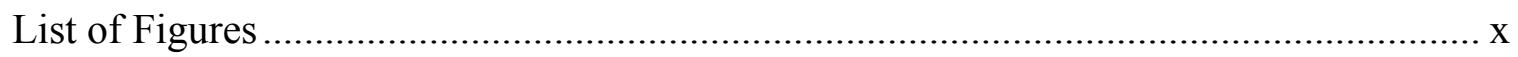

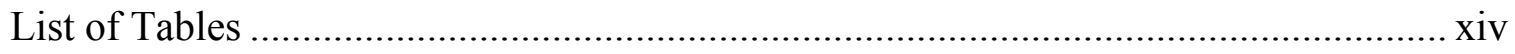

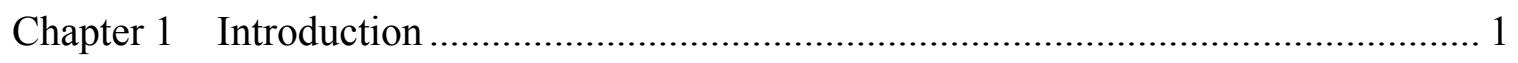

1.1 Background of Inertial Body Sensor Networks ......................................... 2

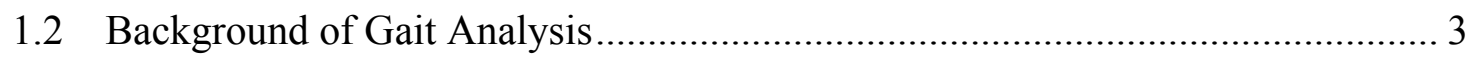

1.3 Vision of Inertial Body Sensor Networks for Mobile Gait Analysis ................... 8

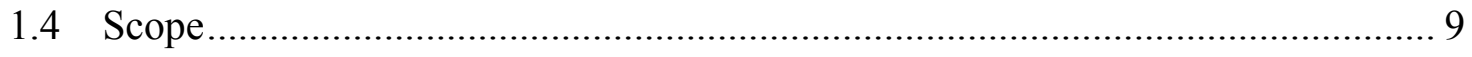

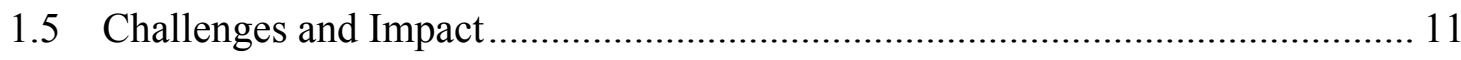

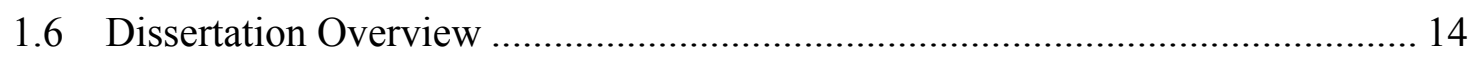

Chapter 2 Kinematic Information Extraction ....................................................... 16

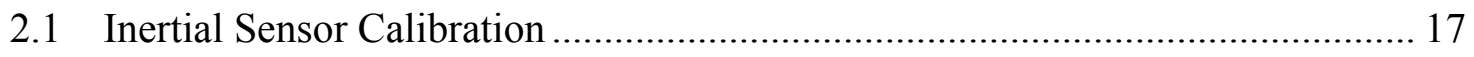

2.1.1 Reference Based Accelerometer Calibration ......................................... 18 
2.1.2 Non-Reference System Based Accelerometer Calibration .......................... 19

2.1.3 Turntable Based Gyroscope Calibration ................................................... 20

2.1.4 Angle Based Gyroscope Calibration........................................................... 21

2.2 Synchronization for Multi-node Application .................................................. 22

2.2.1 Event-based Synchronization........................................................... 23

2.2.2 Time Transmission Based Synchronization............................................. 24

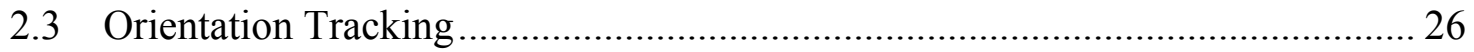

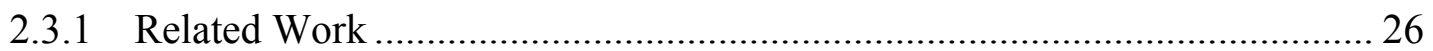

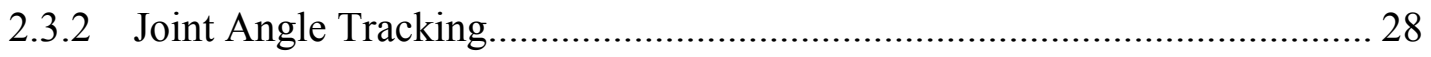

2.3.3 Mounting Calibration............................................................................. 29

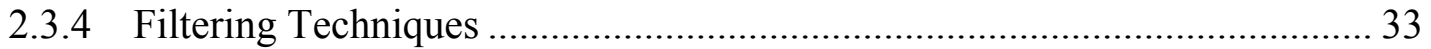

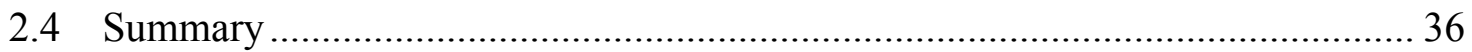

Chapter 3 Linear Analysis for Temporal Feature Extraction..................................... 38

3.1 Gait Specific Temporal Features ................................................................. 39

3.1.1 Gait Cycle Segmentation and Cadence..................................................... 39

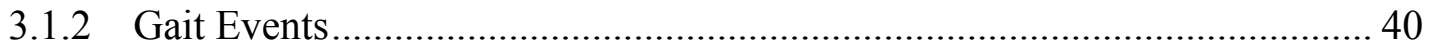

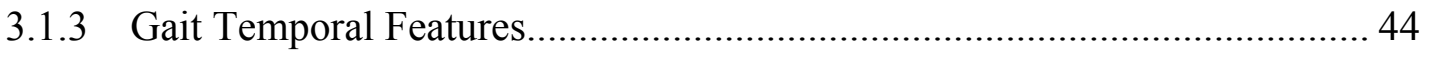

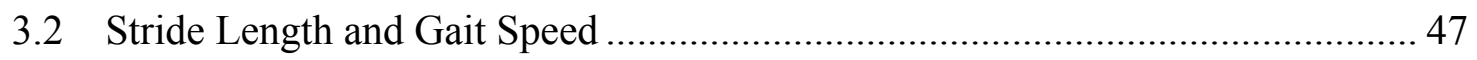

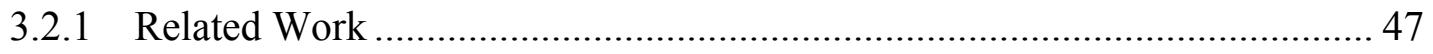

3.2.2 Stride Length Computation.................................................................. 50 
3.2.4 Discussion of Gait Speed Estimation Method using Human Gait Model .... 55

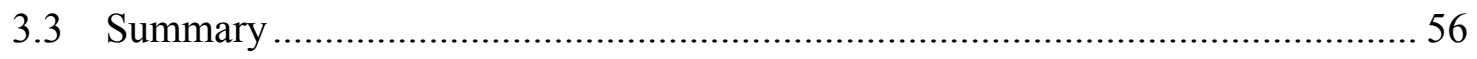

Chapter 4 Nonlinear Analysis for Nonobvious Gait Feature Extraction ...................... 57

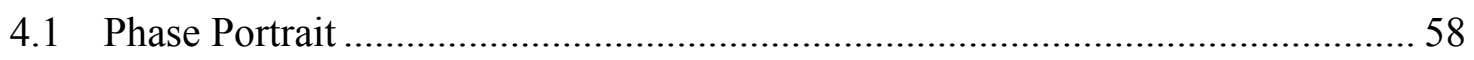

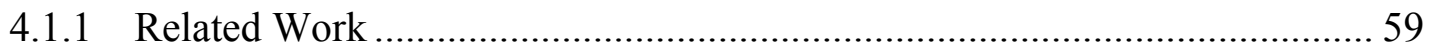

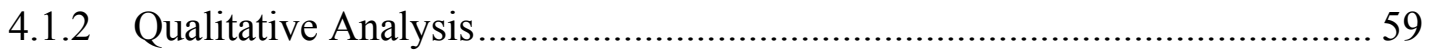

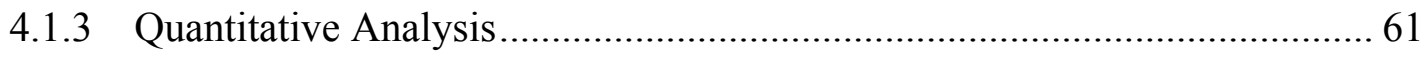

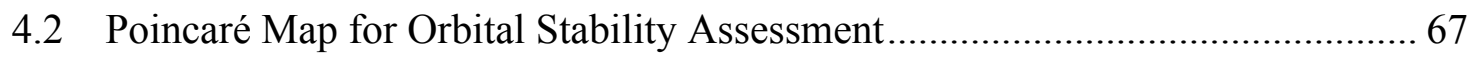

4.3 Lyapunov Exponent for Stability Assessment............................................. 69

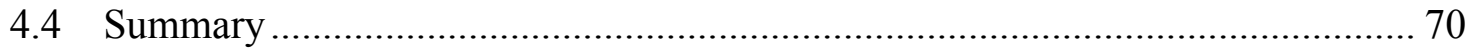

Chapter 5 Introducing Machine Learning Techniques for Feature Extraction ............. 72

5.1 Advantages of Combining Machine Learning with First-Principles Modeling... 73

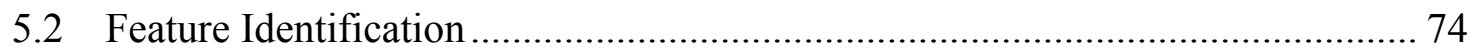

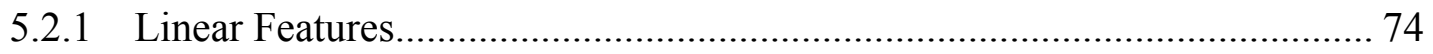

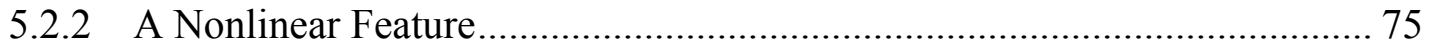

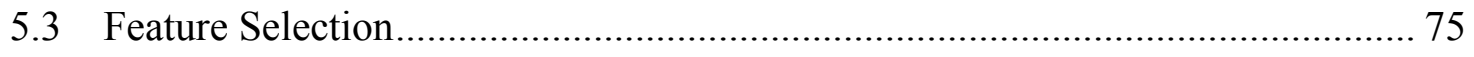

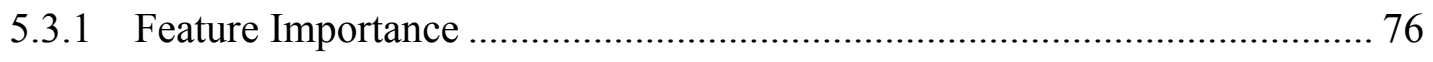

5.3.2 Automatic Feature Selection............................................................... 77

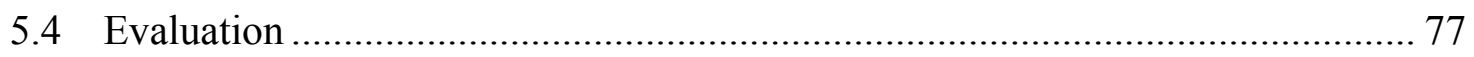


5.4.1 Comparison Based on Machine Learning ................................................ 78

5.4.2 Diagnostics of the Linear Regression Model............................................ 80

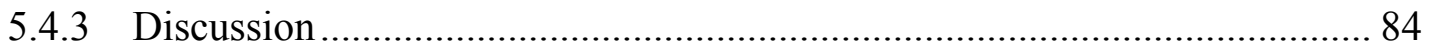

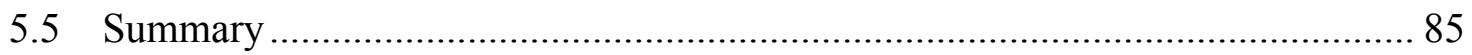

Chapter 6 Case Studies in Medical Research ......................................................... 86

6.1 Orthoses for Children with Cerebral Palsy ................................................... 87

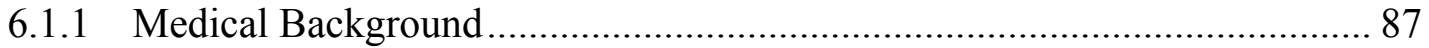

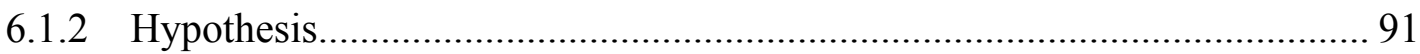

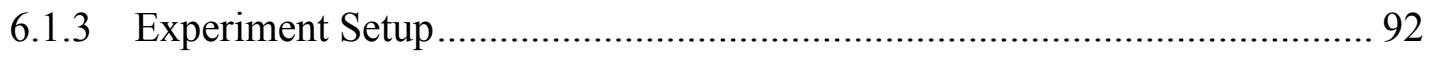

6.1.4 Ankle Joint Angle Extraction............................................................... 98

6.1.5 Other Gait Parameters for Assessing Ankle Foot Orthosis ........................ 99

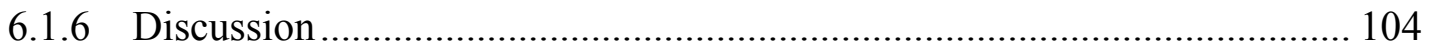

6.2 Aiding Diagnosis of Normal Pressure Hydrocephalus ................................... 104

6.2.1 Medical Background ......................................................................... 104

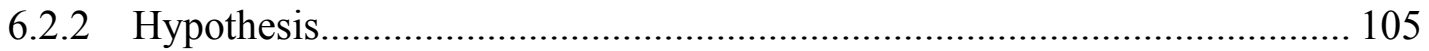

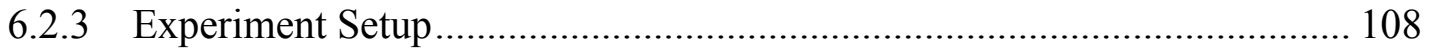

6.2.4 Features Extracted from Clinical Measurement ...................................... 109

6.2.5 Features Extracted from Inertial BSNs ............................................... 111

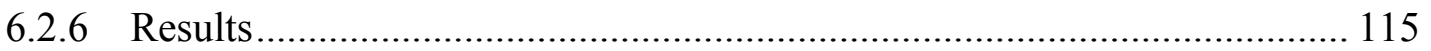

6.2.7 Discussion ................................................................................ 118 
6.3 Early Diagnosis of Multiple Sclerosis ............................................................ 119

6.3.1 Medical Background ................................................................................. 119

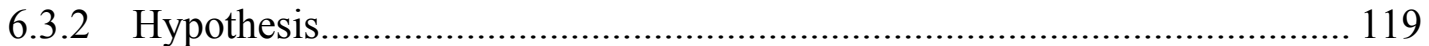

6.3.3 Experiment Setup........................................................................... 120

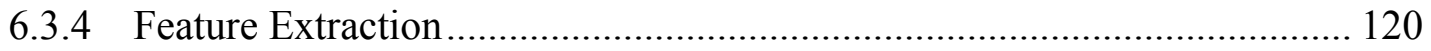

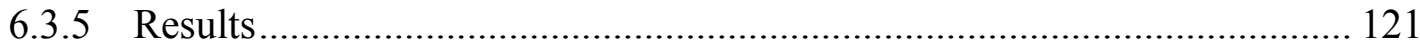

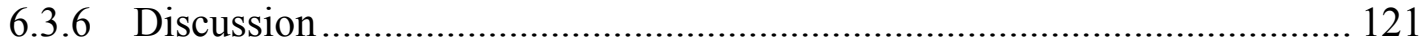

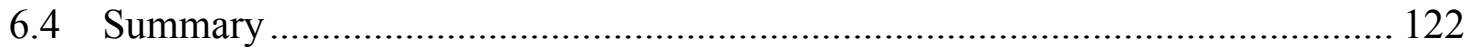

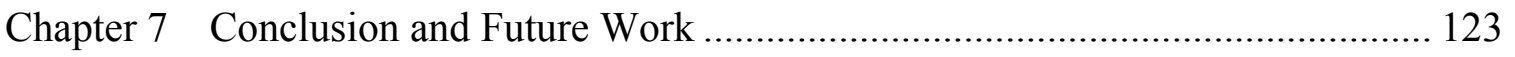

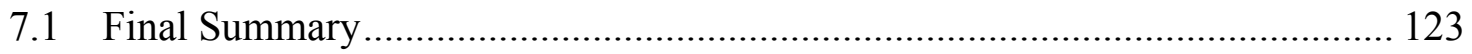

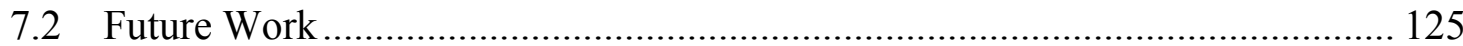

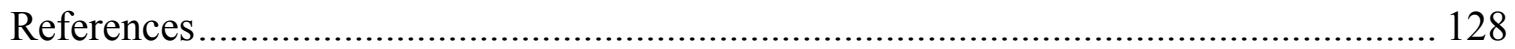

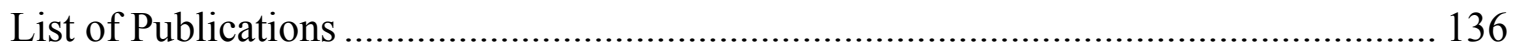




\section{List of Figures}

Figure 1 Panorama of Body Sensor Network (BSN) [2] ……….................................. 2

Figure 2 Several Medical Applications Requiring Gait Analysis....................................... 4

Figure 3 Subject Wearing Reflexive Markers Walking in an Industrial Standard Gait Analysis Instrument -Vicon - Instrumented Gait Lab ……........................................... 6

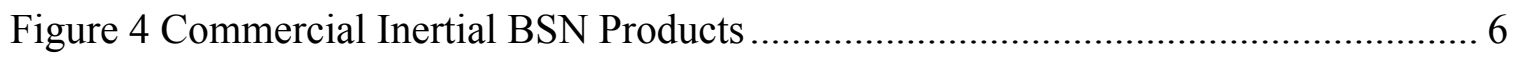

Figure 5 Qualitative Assessment of Current Gait Analysis Situation................................. 7

Figure 6 TEMPO 3.1 Inertial Body Sensor Network (BSN) System ................................ 8

Figure 7 Raw Signals from Inertial BSN ………………....................................... 10

Figure 8 A Turntable for Gyroscope Calibration............................................................... 21

Figure 9 Knee Angle RMSE Due to Desynchronization .............................................. 23

Figure 10 An Inertial BSN Node and Corresponding Axis Orientation............................ 29

Figure 11 Validation of Mounting Calibration Method................................................... 32

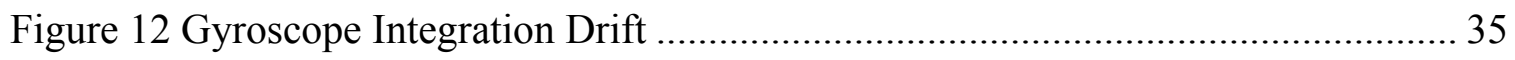

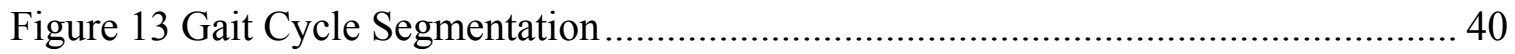

Figure 148 Frame/Second Shots of Gait Events in One Gait Cycle ............................... 42

Figure 15 Gait Events During One Gait Cycle ……………........................................ 43 
Figure 16 Critical Gait Events Detection on Gyroscope Signal ................................... 44

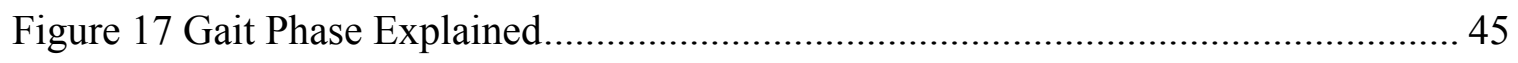

Figure 18 Gait Events in One Cycle's Time Domain Signal...................................... 51

Figure 19 Double Pendulum Model of Gait ............................................................ 52

Figure 20 Subject 1 Gait Speed Estimation Validation .............................................. 54

Figure 21 Subject 2 Gait Speed Estimation Validation ............................................. 54

Figure 22 Shank Phase Portrait of A Health Subject's One Gait Cycle ........................... 60

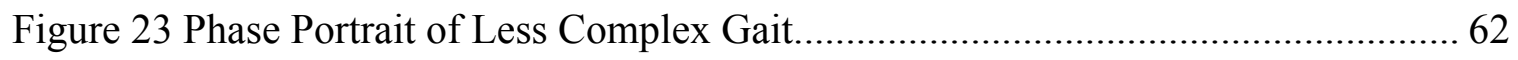

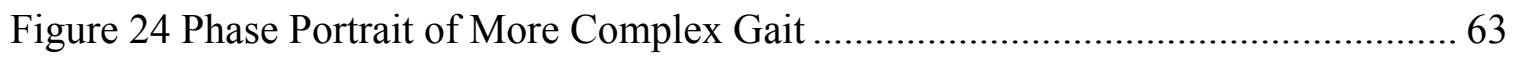

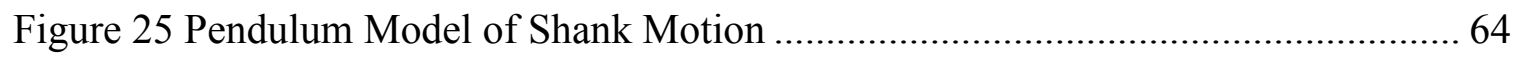

Figure 26 Phase Portraits of A Healthy Subject at Different Gait Speeds...................... 66

Figure 27 Regressed Linear Speed Model by Phase Portrait Area ................................ 66

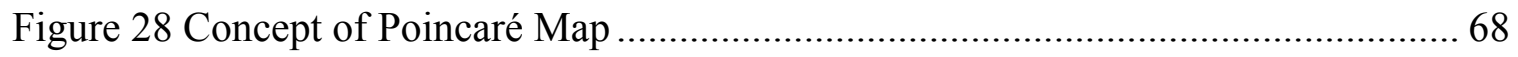

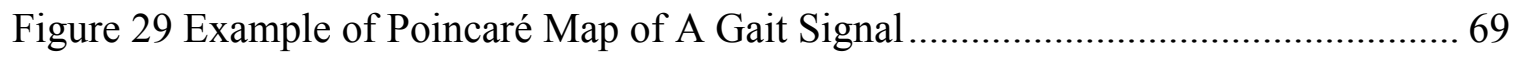

Figure 30 Correlation Coefficients between Different Features and Gait Speed. ........... 76

Figure 31 Intra-subject Validation with Linear Features ........................................ 78

Figure 32 Intra-subject Validation with One Nonlinear Feature ................................ 79

Figure 33 Inter-subject Validation with Linear Regression....................................... 80

Figure 34 Diagnostic Plot of Linear Regression with Linear Features.......................... 81

Figure 35 Diagnostic Plot of Linear Regression with One Nonlinear Feature ................ 82

Figure 36 Diagnostic Plot of Linear Regression with All Features .............................. 83

Figure 37 Children with CP in (A) Equinus and (B) Crouch Gait Deformity................. 87

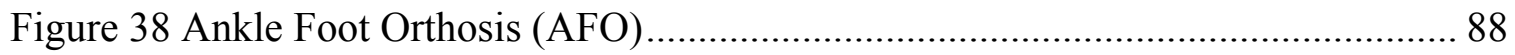


Figure 39 Ankle Joint Angle of CP Gait without and with AFO - the AFO Limits the Range of Ankle Motion 89

Figure 40 Shank Angular Velocity of CP gait without and with AFO - the AFO Helps to Promote a Heel Strike 90

Figure 41 Dorsiflexion and Plantarflexion ............................................................ 92

Figure 42 Healthy Subject Wearing AFO for Experiment ........................................ 93

Figure 43 Ankle Joint Angle of Normal Gait ......................................................... 94

Figure 44 Ankle Joint Angle of Simulated Crouch Gait............................................ 95

Figure 45 Ankle Joint Angle of Simulated Equinus Gait .......................................... 95

Figure 46 Children with CP Instrumented with Inertial BSN Nodes for Experiment...... 97

Figure 47 Ankle Joint Angle of Children with CP Wearing AFO ................................. 99

Figure 48 Average Ankle Angle Range per gait cycle of 4 CP subjects with AFO ....... 100

Figure 49 Health Subject Shank Phase Portrait without AFO .................................... 102

Figure 50 Shank Phase Portrait of CP Subject without AFO ................................... 102

Figure 51 Gait Complexity Assessment of CP Gait ................................................ 103

Figure 52 Differential Diagnosis of NPH in Clinics ............................................. 106

Figure 53 Suspected NPH Subjects Mounted with TEMPO Inertial BSN Nodes.......... 108

Figure 54 Clinical Obtainable Gait Features (a) Gait Speed (b) Step Length and (c) Stride Time Fail to Separate the Suspected NPH Group.................................................. 110

Figure 55 Reconstructed Phase Portraits of Healthy and NPH Subjects ...................... 114

Figure 56 Average Stride Time Standard Deviation Change of Suspected NPH Patients Pre- to Post-HVLP Does Not Provide NPH Separability ......................................... 115 
Figure 57 Average Double Stance Time Change of Suspected NPH Patients Pre- to PostHVLP Does Not Provide NPH Separability 116

Figure 58 LyE Change of Suspected NPH Patients Pre- to Post-HVLP Does Provide NPH Separability (NPH patients improved post-HVLP (i.e., lower LyE), while non-NPH patients deteriorated), Indicating Its Potential to Aid in NPH Diagnosis ..................... 117

Figure 59 Effect Size Measured by Cohen's D of Some Gait Features........................ 121

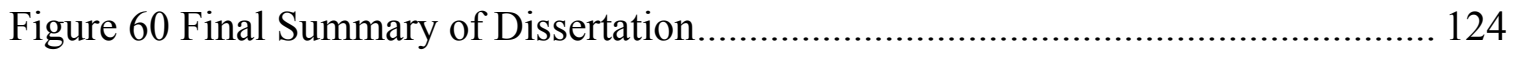

Figure 61 Inertial BSN Enabled Mobile Gait Analysis in Natural Environment ........... 126 


\section{List of Tables}

Table 1. Angles measured at various mounting errors. ............................................... 33 


\section{Chapter 1}

\section{Introduction}

Inertial Body Sensor Networks (BSNs) have a promising prospect for bridging the gap between the requirement of high precision, portable gait analysis in medical research and clinical practice and the lack of such measurement tools in industry nowadays. By sensing the angular velocity and linear acceleration of human body segments where they are mounted on, inertial BSNs observe and record human motion in the fashion of derivatives of spatial information within an inertial frame. In order for these raw signals collected from inertial BSNs to be interpretable, meaningful information must be extracted and analyzed with insight to benefit medical research. Centered around the key question: how to transform the raw signals to useful information for gait analysis, this chapter introduces the background of BSN technology and gait analysis, the vision of applying inertial BSNs to gait analysis and the research questions induced from such vision. 


\subsection{Background of Inertial Body Sensor Networks}

Body Sensor Networks (BSNs) have excited research with a great potential for continuously and quantitatively monitoring human physiology, kinesiology, psychology and neuropsychology etc., [1] as illustrated in Figure 1.

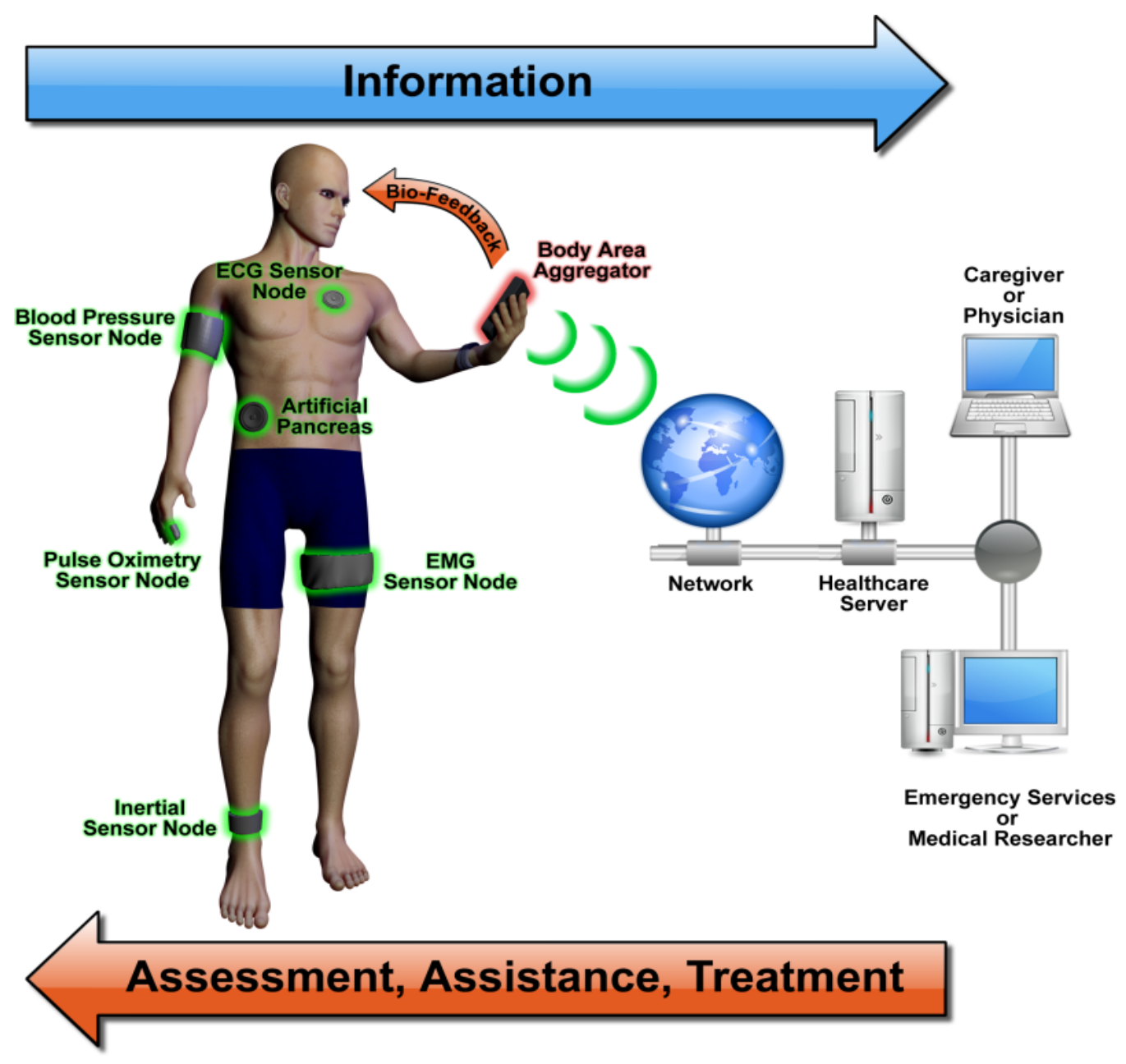

Figure 1 Panorama of Body Sensor Network (BSN) [2]

Inertial BSNs are one type of BSNs that measures the angular velocity and linear acceleration of the body segment where they are mounted on. Research interest using 
inertial BSNs mainly focuses on motion capture and gait analysis. While the more general term 'motion capture' is adopted more by augmented virtual reality in video gaming system and (e.g. Wii ${ }^{\circledR}$, Kinect ${ }^{\circledR}$ and various smartphone apps utilizing accelerometers and gyroscopes) and entertainment industry, it is also being used in biometric security (e.g. handwriting recognition [3] and gait identification), posture monitoring [4], daily activity monitoring [5][6], athletic kinesiology and exercise physiology. Under this background, the opportunity of adopting inertial BSNs for various gait analysis involved medical applications is especially exciting and promising.

\subsection{Background of Gait Analysis}

Gait analysis has been an established research area for various medical and healthcare applications. Through studying locomotion of human beings, these applications range from direct ones such as identifying and monitoring gait pathology and degradation, assessing the efficacy of the orthoses or prosthetics [7] (Figure 2 (a) and (b)), assessing fall risks and fall prevention [8] (Figure 2 (d)), evaluating the rehabilitation treatment (e.g. for knee surgery or stroke recovery), to non-obvious ones such as aiding diagnosis of gait degradation (Figure 2 (c)) manifested neuropathy and assessing the medical intervention for those pathologies [9][10][11] [12]. 


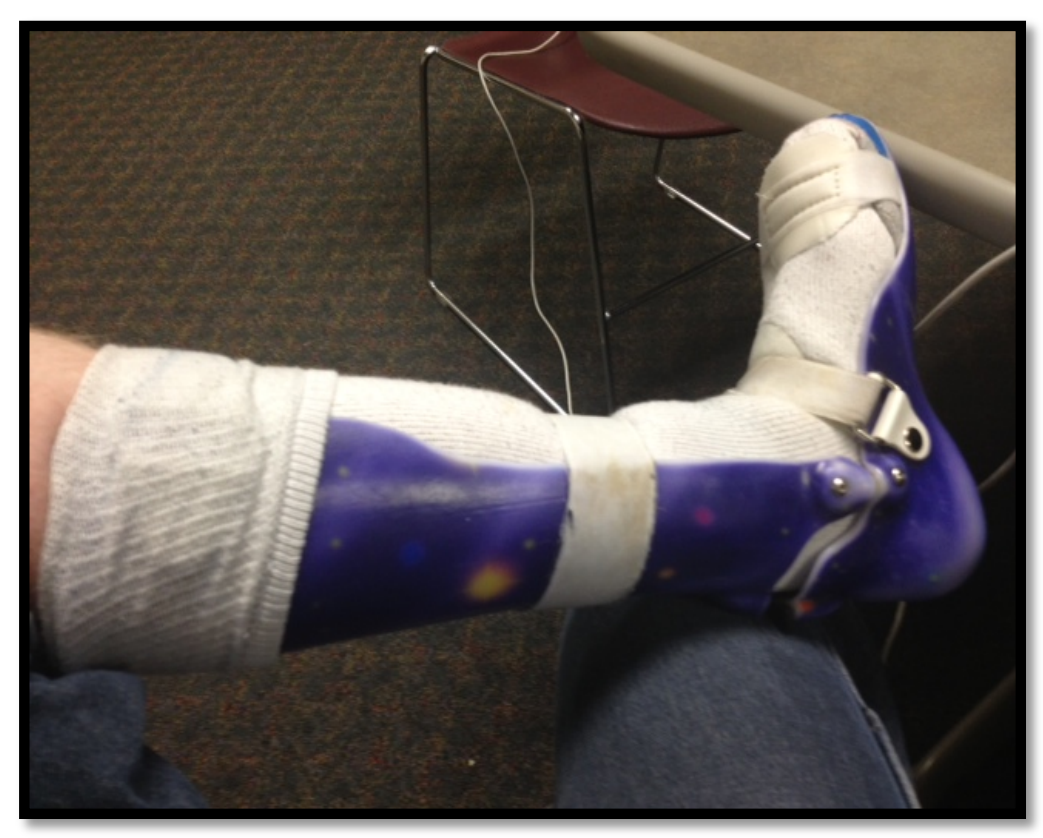

(a)

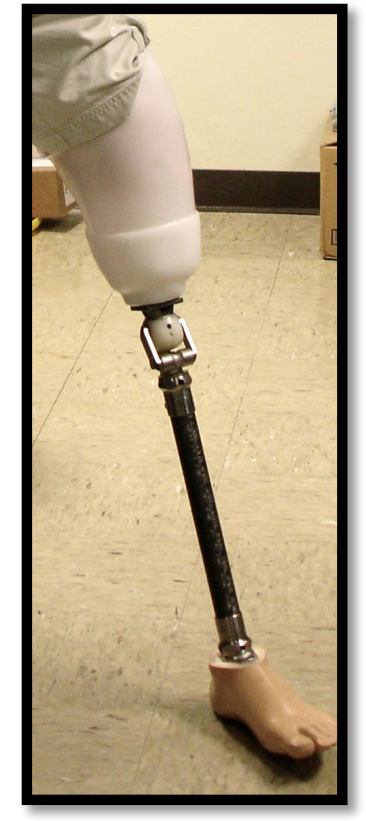

(b)
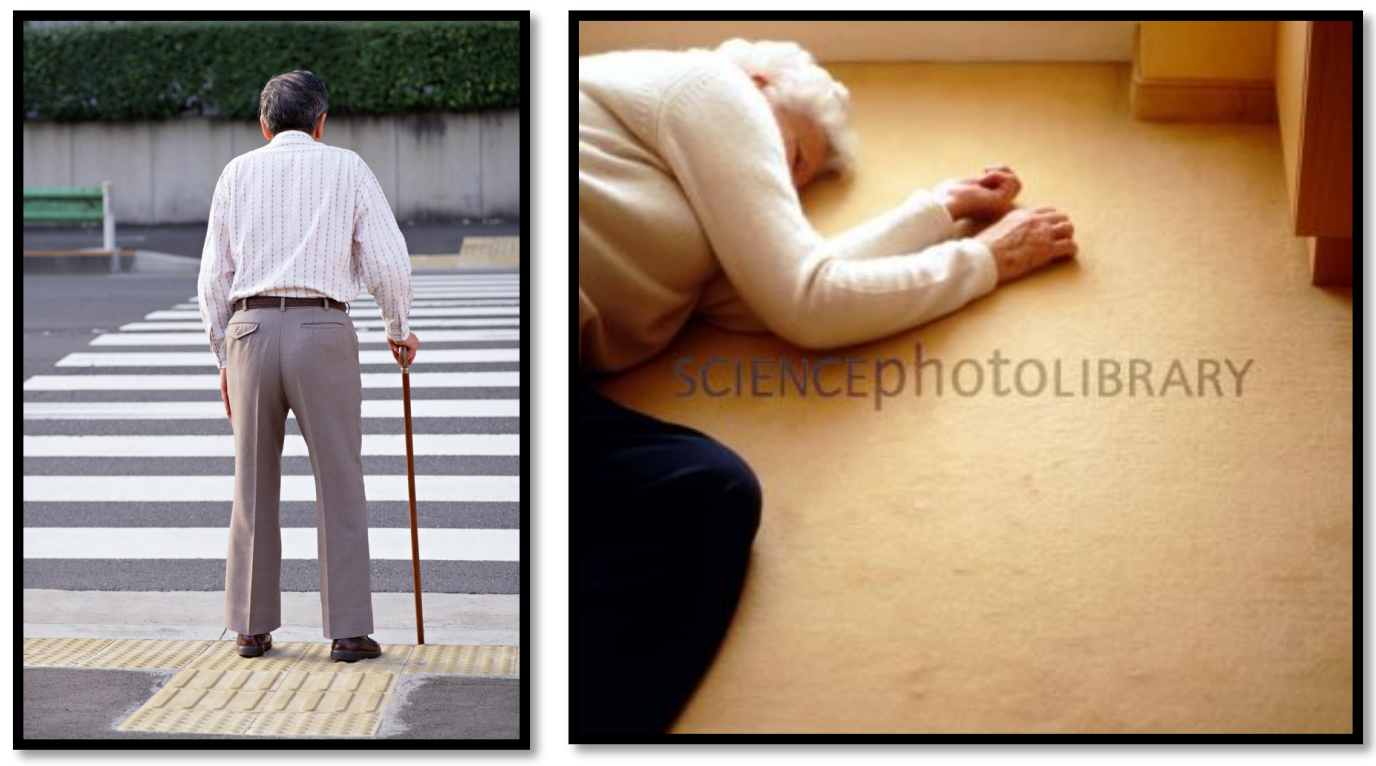

(c)

(d) source: Cristina Pedrazzini/Science Photo Library

Figure 2 Several Medical Applications Requiring Gait Analysis 
The quality and validity of these gait analysis applications can highly depend on the measurement instrument used in the analysis. On one hand, as important to other medical instrument, gait analysis instrument also requires high precision and high accuracy for a number of gait parameters (e.g., gait speed, joint angle, stride length, gait cycle, etc.). On the other hand, most of these gait analysis applications would benefit from continuous, longitudinal observation.

However, in current clinics, gait analysis is usually done with subjective and qualitative approaches such as clinical observation and patient self-report. Some clinics have adopted the industrial standard gait analysis tool- optical motion capture system such as Vicon ${ }^{\circledR}$ (Figure 3) [13]. With infrared cameras capturing the reflexive markers defined human skeleton outline, this kind of systems tracks position information and human motion, and provide high-precision data at a sampling rate of $120 \mathrm{~Hz}$. However, although such systems can deliver highly accurate human movement analysis, these expensive systems require expert operation and are limited to in-lab monitoring; thus the knowledge obtained by such in-lab instruments may not reflect reality. However, such tools is usually expensive, require dedicated lab and expert operation. It also involves invasive and cumbersome marking setup procedure, rendering it less practical for clinical use and unnatural for the human subject.

Modern pedometers and activity monitors (Figure 4) present another scenario. These portable commercial products which could provide longer-term monitoring, but could only provide coarse information such as steps one took per day or distance travelled 
based on the steps information, which do not suffice the high precision requirement for medical research.

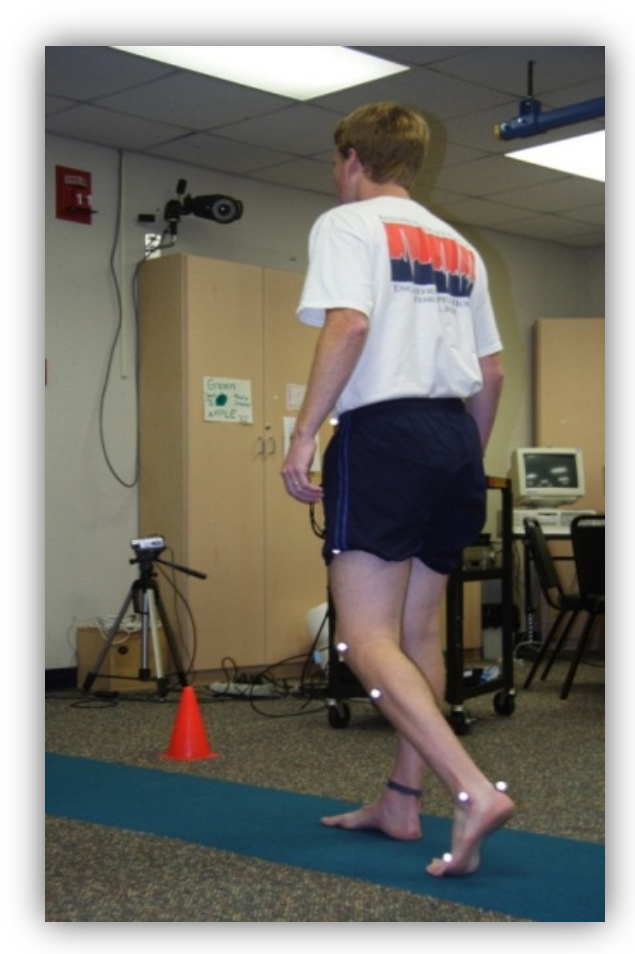

Figure 3 Subject Wearing Reflexive Markers Walking in an Industrial Standard Gait Analysis Instrument -Vicon - Instrumented Gait Lab

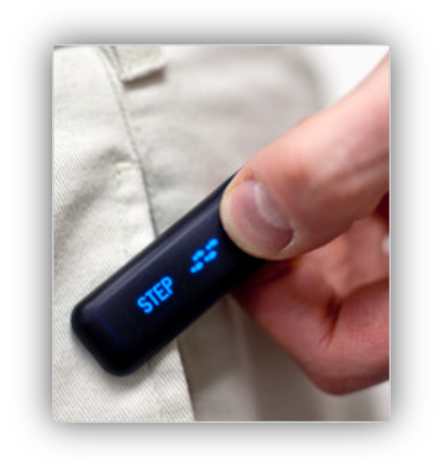

(a) Fitbit ${ }^{\circledR}$ (source:nytimes.com)

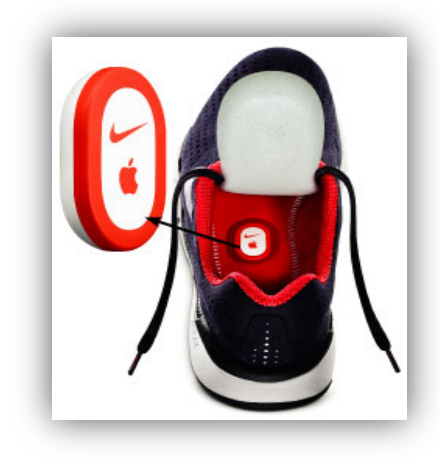

(b) Nike+ ${ }^{\circledR}$ (source: solution.allthingsd.com) 


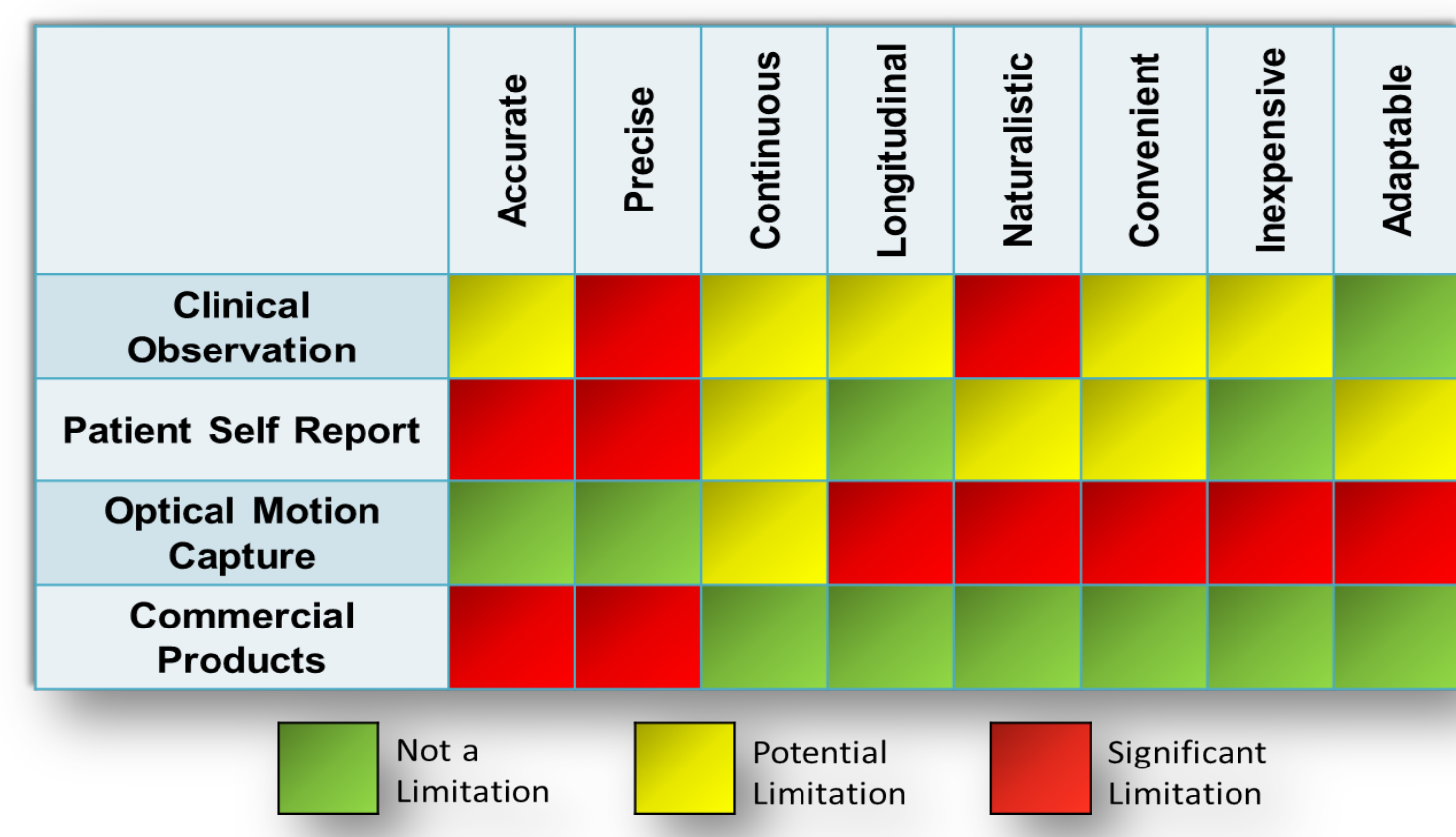

Figure 5 Qualitative Assessment of Current Gait Analysis Situation

These situations in gait analysis can be best summarized in Figure 5. While clinical observation and patient self-report lack in accurate, quantitative measure, the modern tools such as optical capture system and commerical products either lacks precision or portability. For gait analysis to be realized in the real world, measurement tools suffice both high precision, high-fidelity motion tracking requirement and continuous, out-of-lab requirement are necessary. 


\subsection{Vision of Inertial Body Sensor Networks for Mobile}

\section{Gait Analysis}

The emerging wireless inertial BSNs naturally provide a non-invasive wearable solution, enabling gait analysis to be realized in the real world. For such medical applications, inertial BSNs not only could function as an easy-to-use and economical tool for quantitative medical observation, but also could enable out-of-lab and long-term monitoring assessment to clarify the previous unclear medical conclusion based on in-lab and/or qualitative clinical observation and further prompt medical research.

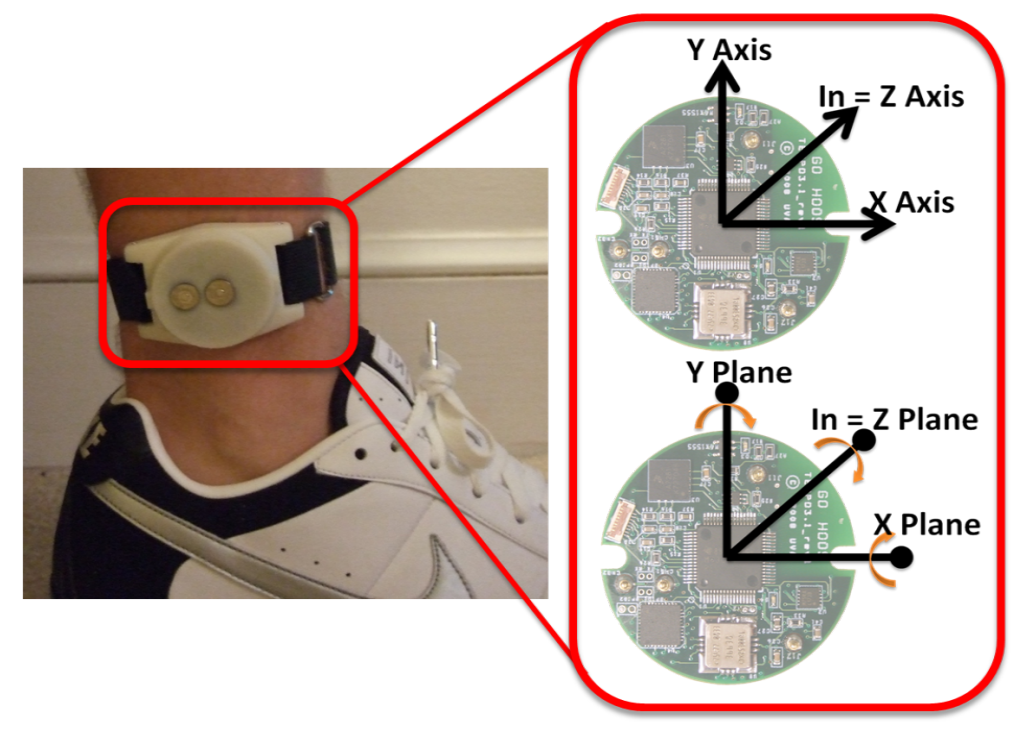

Figure 6 TEMPO 3.1 Inertial Body Sensor Network (BSN) System

The TEMPO system [14] (Figure 6) developed by the INERTIA Team at the University of Virginia, along with a number of medical collaborators, is such an inertial BSN system including 3-axis accelerometer and 3-axis gyroscope sensors and providing six-degree- 
of-freedom motion sensing in a form factor of a form factor of wrist watch. This platform will be used as the research platform in the projects described in this dissertation.

\subsection{Scope}

Many challenges obstruct the futuristic vision of inertial BSN enabled portable gait analysis, such as form factor, power consumption, information extraction, wireless communication, and real-time feedback control. This dissertation in particular, focuses on only the information extraction aspect. Since first and foremost, the inertial BSN technology must be accepted by the medical world before it can further empower and propel medical research, and information extraction and knowledge discovery is a critical bridge connecting the engineering world and medical world. And to vitalize real-world deployment of this technology, this step will further produce the intelligence to be delivered and stored.

First, to have the medical field accept the technology, data must be presented in a communicable way for the medical staff and healthcare givers. Due to the computation complexity of the feature extraction, data obtained from inertial sensors, however, are usually raw signals (e.g. acceleration, angular velocity etc., shown in Figure 7). Yet it is essential to extract information and transform them into knowledge in order for the data to tell certain human behavior or gait pattern. To unveil such information behind the signals, great signal processing efforts with the background of inter-disciplined knowledge are required. 


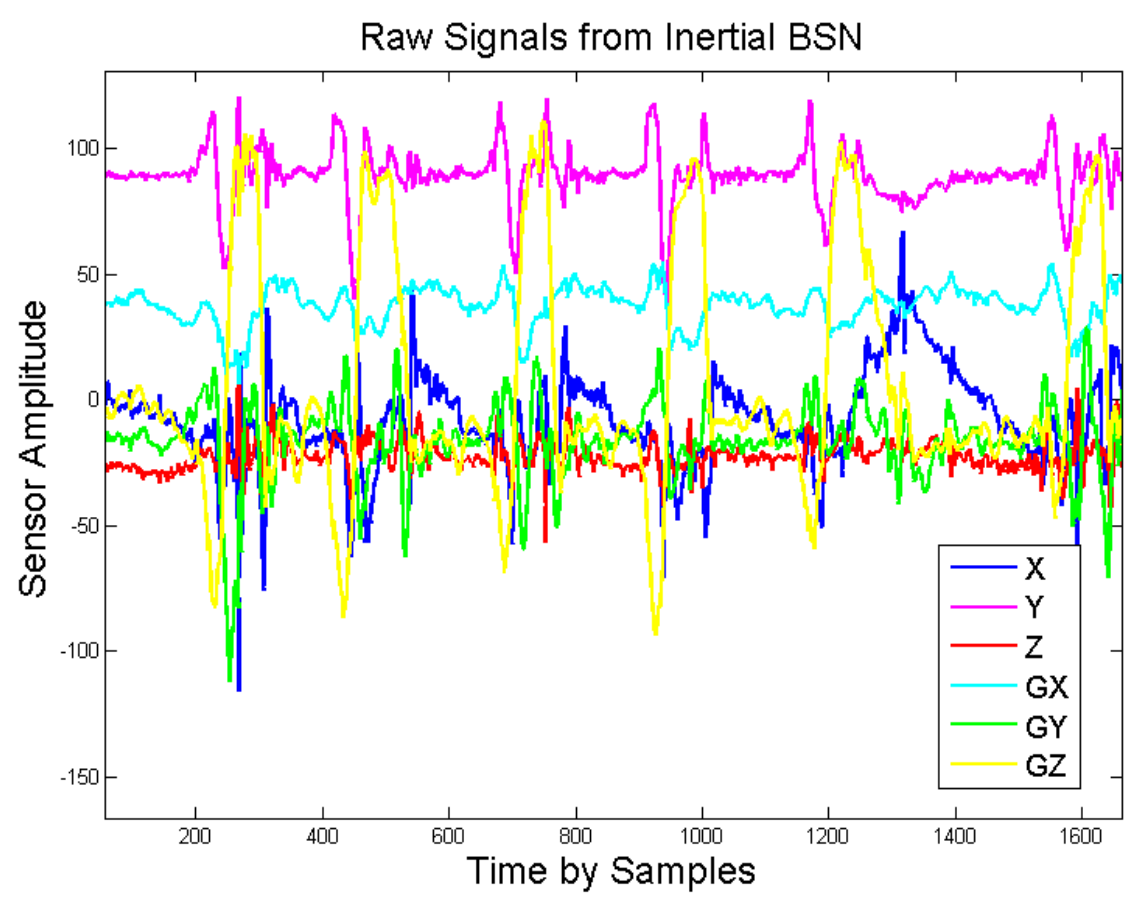

Figure 7 Raw Signals from Inertial BSN

Second, storing the right and meaningful representation of data is essential to data storage. While time series is naturally to be stored due to the monitoring function of inertial BSNs, the sample-by-sample kinematic information occupies much storage with providing little knowledge about gait. For example, an application for collecting ankle joint angle requires four inertial BSN nodes. Each node takes 3-axis accelerometer data and 3-axis gyroscope data. To capture subtle motion, all the axes are sampled at $128 \mathrm{~Hz}$. To convert the analog signal to digital data, a 12 bits analog-to-digital convertor (ADC) is used, while each sample contains 2 bytes of data in storage. If all the data were to store in the format of raw ADC values, then after one week's continuous data collection, four nodes data would occupy: 4 (number of inertial BSN nodes) x 6 (number of axes/ node) x 2 (bytes/sample) x 128 (samples/second) x 60 (seconds/minute) x 60 (minutes/hour) x 24 
(hours/day) x 7 (days/week) $=3.46$ Gigabytes $(\mathrm{GBs})$ ! Thus one subject's yearly data $(180$ GBs) in this format could quickly saturate a computer hard drive (usually around 300 GBs). It would be extremely troublesome to dive into this ADC data ocean and find the useful information. Therefore, to be able to reuse and research on the continuously and longitudinally collected data efficiently, one must develop techniques for data compression and organization. Extracting useful gait features targeting application requirement is potentially an important approach to solve this problem. However, this dissertation will focus on the knowledge discover scope and leave the data reduction question for further research.

\subsection{Challenges and Impact}

The background mentioned above infers that gait feature extraction has become critical to the vitality of technology using inertial BSNs for medical applications. Therefore, to demonstrate the feasibility and importance of inertial BSNs to medical applications, this dissertation will undoubtedly center on the key question "How to best and most efficiently transform the raw inertial sensor data to useful information and medical knowledge in gait analysis?"

Many paths exist between the input (e.g. raw inertial signals) and the output (e.g. useful information and medical knowledge) - inadvertently complicating the answers. Usually, there are two approaches to tackle this problem: first-principles model based and machine learning based. This dissertation mainly focuses on the former approach and explores 
ways to combine the latter approach as well. However, there are many challenges associated with both approaches.

For example, while these sensors provide rich information on the kinematics of human motion, the accurate spatial information required for many gait parameters is challenging to obtain. Intrinsic sensor noise causes integration drift, the uncertainty of mounting nodes on human bodies causes a systematic bias, and movement in multiple planes causes computational errors, all of which hinder the acquisition of accurate spatial features such as joint angle, stride length and gait speed. Moreover, current state-of-art feature extraction has focused on extracting accurate kinematic information by conquering spatial-information extraction challenge, and the extraction of gait features in the context of real applications behind the kinematic time series are rather less explored - lacking the evidences of adopting the information extracted from inertial BSNs for medical research.

Meanwhile, the to-be-enabled long-term, natural environment monitoring also creates 'big data' sets by data collection in a less controlled fashion, demanding the efficient gait feature extraction and selection for data storage and decision making given specific medical applications. Moreover, despite the reported success in extracting the desired gait features, the prevailing techniques in literature lack a systematic approach of extracting gait features. Therefore, it is desirable to have a systematic way in order to extract the gait features efficiently.

To address these issues, several research questions are posed as follows: 
- How to use a general, systematic approach to identify meaningful gait features targeting a specific medical application?

- How to extract those gait features from inertial BSNs?

- How to leverage knowledge from other domains for this interdisciplinary research?

- How to adopt machine learning to systematically correcting extraction accuracy and automating the feature extraction process?

To answer these research questions, this dissertation presents a roadmap of extracting gait features for gait analysis related medical applications. First, techniques to tackle the challenge of tracking accurate spatial information in order to provide accurate kinematic information will be introduced. Given the accurate kinematic information, linear analysis of extracting time domain gait features will be applied. This work also explores the opportunity of leveraging biomechanics and applying nonlinear analysis, in order to refine signal processing and enhance data separability and interpretability from features extracted from nonlinear analysis. Also, machine learning approaches will be explored for automatic solution to segmenting long period of sensor data and feature selection and reduction. Lastly, various case studies for applying these techniques to different medical applications will be discussed to exemplify the systematical approach.

By summarizing existing techniques and leveraging biomechanical knowledge and related medical domain knowledge, overall, this research is aimed to provide an organized guideline for extracting gait features from inertial BSN data and further transform these features into useful medical knowledge, demonstrating the vision of 
using inertial BSNs for longitudinal study to many related medical applications. The outcomes of this research are expected as following:

- Establish the feasibility of BSN gait analysis for medical applications

- Provide a roadmap for gait feature extraction, selection, and data reduction from inertial BSN data

- Validate the accuracy of extracted gait features against gold standard technologies or ground truth

- Demonstrate efficacy of extracted gait features in several medical case studies

\subsection{Dissertation Overview}

Given the outlook and impact of this research, the thesis of this dissertation can be stated as follows:

This research establishes a systematic approach to extracting gait features from inertial body sensor networks. Informed by biomechanics and combining machine learning techniques and modeling from first principles, this approach provides an effective framework whose utility is validated via a series of medical case studies.

The rest of this dissertation is organized as follows. After the introduction of Chapter 1, the technique details of extracting accurate kinematics are presented in Chapter 2. Then common features that can be extracted from linear analysis are detailed in Chapter 3 including time domain features and gait cycle based features. Chapter 4 studies several commonly used nonlinear analysis tools for inertial BSN data, including qualitative and 
quantitative phase portraits analysis, applying Lyapunov exponent for stability analysis, and applying Poincaré map for regularity analysis. Chapter 5 explores how to utilize machine learning techniques for gait feature extraction and exemplify the concept by extracting gait speed. Chapter 6 will apply the features extracted from Chapter 3-5 to several medical applications as case studies. Chapter 7 will conclude the dissertation and layout the future work. 


\section{Chapter 2}

\section{Kinematic Information Extraction}

Kinematics can be considered as the most basic time series one could obtain by using inertial BSNs, due to the two sensing modalities the currently available inertial sensors provide: acceleration by accelerometer and angular velocity by gyroscope, respectively. As most current inertial sensors are analog sensors, the data obtained from the sensor is usually Analog-to-digital Converter (ADC) values, which need proper conversion to be transformed to the physical values. How to convert the ADC values, and what other time series can be produced from the physical values become the focus of this chapter. By devising a set of procedures, including calibration, synchronization, mounting calibration, geometric transformation, and drift canceling, this chapter aims to transform the raw ADC sensor values to kinematic information accurately, producing the primary information in the gait feature extraction system.

\footnotetext{
This chapter is partially adapted from publication [P11] and [P12]

${ }^{\dagger}$ The work in this section is attributed to my teammate Jeff Brantley.
} 


\subsection{Inertial Sensor Calibration}

Accelerometers and rate gyroscopes used in inertial BSN platforms are typically assumed to follow a linear model. The sensitivity is the ratio of voltage change to change in the physical quantity, and the offset is the output voltage when no motion (or gravitational field, in the case of accelerometers) is applied to the sensor. The relationship between the sensed quantity $(M)$, and the output voltage $(V)$, is described by Equation (1), which contains two key parameters: the sensitivity $(S)$ and the offset $(O)$.

$$
M=\frac{(V-O)}{S}
$$

The sensitivity is the ratio of voltage change to change in the physical quantity, and the offset is the output voltage when no motion (or gravitational field, in the case of accelerometers) is applied to the sensor. These parameter values can be found in the sensor datasheets, but in practice they will deviate from the expected value due to various sources of errors.

These sources of errors include manufacturing variations and environmental conditions, such as temperature, meaning that sensitivity and offset will vary both across different sensors at a given time, or within a single sensor at various times. Datasheets and white papers from manufacturers additionally list other possible error sources, such as nonlinearity, non-orthogonality between axes, and cross-axis sensitivity. Finally, variations in chip mounting on a printed circuit board (PCB) or mounting of the PCB in the node packaging can place the sensing axes slightly out of the assumed frame of reference. The sensitivity is the ratio of voltage change to change in the physical quantity, and the offset 
is the output voltage when no motion (or gravitational field, in the case of accelerometers) is applied to the sensor.

Therefore, it is impractical to individually compensate for these sources of errors, and so some form of calibration is employed to holistically minimize their effect. The sensor response is checked against a known reference point (such as the gravitational field or a turntable) in order to calculate the actual sensitivity and offset by linear mapping or Newton's method [15][16][17][18]. In the following sections, methods for calibrating accelerometer and gyroscope sensors are detailed.

\subsubsection{Reference Based Accelerometer Calibration}

Reference based accelerometer calibration requires level surface or inclinometer to provide physical ground truth as mapping reference. The simplest calibration technique is the linear mapping of two points measured with respect to gravity into the linear function. For accelerometers, each axis of sensor is exposed to $1 \mathrm{~g}$ and $-1 \mathrm{~g}$ by placing it parallel with gravity. The sensitivity and offset are calculated from these reference points according to Equation (2).

$$
\left\{\begin{array}{l}
S=\frac{V_{\max }-V_{\min }}{2} \\
O=\frac{V_{\max }+V_{\min }}{2}
\end{array}\right.
$$

With S and O obtained, Equation (2) can be applied to convert the ADC value directly into $g$. 


\subsubsection{Non-Reference System Based Accelerometer Calibration}

Non-reference system based accelerometer calibration does not require a particular reference instrument such as inclinometer. However, it does not mean the calibration can be carried without reference. In fact, it implicitly uses the reference of an important principle of accelerometer: whenever the accelerometer is stationary on earth, the sum of vector magnitude of 3 axes is $1 \mathrm{~g}$. This principle is described by Equation (3),

$$
\sqrt{A_{x}^{2}+A_{y}^{2}+A_{z}^{2}}=1
$$

Equivalently,

$$
A_{x}^{2}+A_{y}^{2}+A_{z}^{2}=1
$$

With this constant, we can set up a series of equations to mathematically estimate the variables in the equations. As for each axis, the sensitivity and offset are constant but unknown. And the goal of calibration is to obtain the constant and offset. With this in mind, we rewrite Equation (4) as:

$$
\left(\frac{V_{x}-O_{x}}{S_{x}}\right)^{2}+\left(\frac{V_{y}-O_{y}}{S_{y}}\right)^{2}+\left(\frac{V_{z}-O_{z}}{S_{z}}\right)^{2}=1
$$

where $V_{x}, V_{y}, V_{z}$, are the known $\mathrm{ADC}$ readings from the $\mathrm{X}, \mathrm{Y}$, and $\mathrm{Z}$ axis, and $S_{i}$ and $O_{i}$ are the unknown sensitivity and offset, respectively, of axis $x, y, z$. Since there are 6 variables in Equation (5), 6 equations are needed to solve the variables. This means 6 sets of ADC readings, $V_{x}, V_{y}, V_{z}$, are needed from placing the accelerometer in 6 arbitrary 
orientations. (subjected to the gravitational field in six distinct, but arbitrary orientations. To solve the system of quadratic equations expressed as Equation (6), numerical methods such as Newton-Raphson method can be used to obtain the sensitivities and offsets.

$$
F\left(S_{x}, S_{y}, S_{z}, O_{x}, O_{y}, O_{z}\right)=\left(\frac{V_{x}-O_{x}}{S_{x}}\right)^{2}+\left(\frac{V_{y}-O_{y}}{S_{y}}\right)^{2}+\left(\frac{V_{z}-O_{z}}{S_{z}}\right)^{2}-1=0
$$

The non-reference based calibration can be very useful when perfect level surface is not able to obtain. For example, when TEMPO nodes are molded into orthopedic devices, it is no longer easy to align the nodes in parallel or orthogonal to gravity direction. At this point, non-reference based method only requires six distinct positions without requiring the perfect alignment. However, the disadvantage is that the iterative estimation limits the computation to offline post processing from a resource constrained BSN platform.

\subsubsection{Turntable Based Gyroscope Calibration}

Similarly as accelerometer calibration using references of gravitational direction, rate gyroscope sensors can be placed on a turntable and subjected to the available rotational rates, typically 33 RPM and 45 RPM, as shown in Figure 8. 


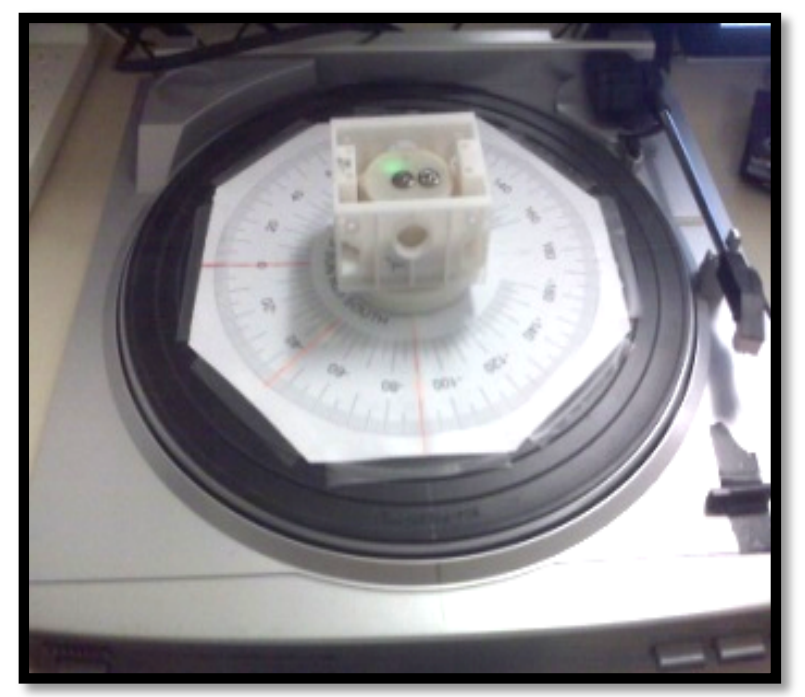

Figure 8 A Turntable for Gyroscope Calibration

$$
\left\{\begin{array}{l}
S=\frac{V_{\max }-V_{\min }}{2 \times 200} \\
O=\frac{V_{\max }+V_{\min }}{2 \times 200}
\end{array}\right.
$$

With S and O obtained, Equation (7) can be applied to convert the ADC value directly into degree/s.

\subsubsection{Angle Based Gyroscope Calibration}

Unfortunately, the mathematical estimation based calibration technique only applies for accelerometers. For gyroscope, there is no implicit ever-present reference as the gravity factor in accelerometer. However, we could use more accessible reference system other than turntable or systems providing constant rotation rate to free the calibration from turntable setup - which is mapping the gyroscope sensor's sensitivity and offset parameters to angles, as presented in Equation (8). 


$$
\left\{\begin{array}{c}
\int_{0}^{t 1} \frac{\omega_{x 1}-O_{x}}{S x} d t=\text { Angle }_{1} \\
\int_{0}^{t 2} \frac{\omega_{x 2}-O_{x}}{S x} d t=\text { Angle }_{2} \\
\vdots \\
\int_{0}^{t n} \frac{\omega_{x n}-O_{x}}{S x} d t=\text { Angle }_{n}
\end{array}\right.
$$

Equation (8) presents the idea of mapping x-axis gyroscope's parameters -sensitivity $(S x)$ and offset $\left(O_{x}\right)$ - to angles $\left(\right.$ Angle). Since within time, the angular velocity $\left(\omega_{x}\right)-$ which is the ADC recording from inertial BSN data - can be integrated into a certain angle as the gyroscope swings. Knowing the precise time of angular motion (integration span) and the angle, we can solve this linear equation, and obtain the two unknowns sensitivity $(S x)$ and offset $\left(O_{x}\right)$. In practice, this process can be achieved by turning the gyroscope on a goniometer in order to find the angle the gyroscope has been turned to. To obtain better accuracy, this process can be repeated in order to eliminate the experiment error.

\subsection{Synchronization for Multi-node Application ${ }^{\dagger}$}

BSN applications employing data fusion across multiple sensor nodes can suffer significant error if not properly time-synchronized. Computations combining nonstationary data sample-by-sample from various nodes will, for a given time lag among nodes, incur error related to the rate of change in the signals. For example, a joint angle

\footnotetext{
${ }^{\dagger}$ The work in this section is attributed to my teammate Jeff Brantley.
} 
computed from two limb segment angles will combine segment angles taken at different times and different postures; the severity of this difference is a function of the time lag between signals and the speed of the limb motion. Figure 9 captures how the accuracy of desired information - knee joint angle - is distorted with simulated synchronization lag. The maximum RMSE can exceed 20 degrees, which is greatly below the medical application precision requirement (less than 5 degrees RMSE).

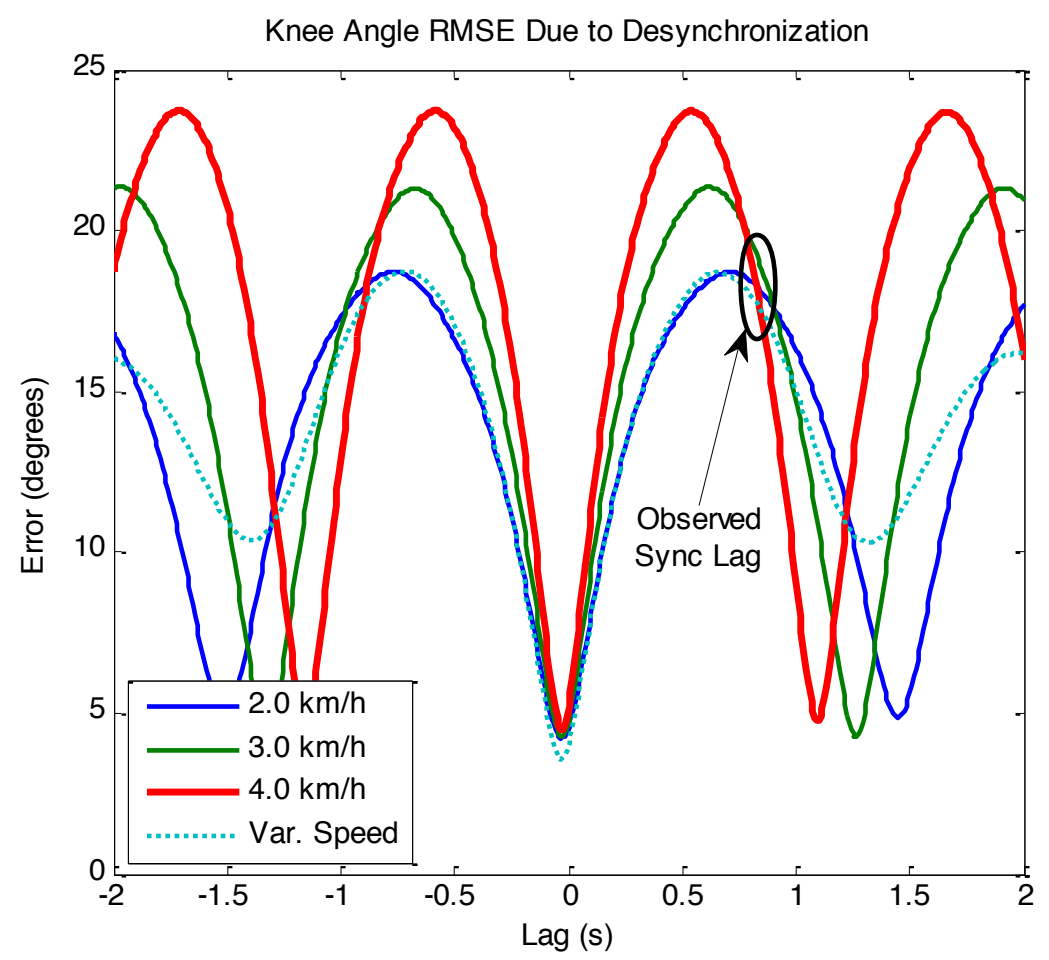

Figure 9 Knee Angle RMSE Due to Desynchronization

\subsubsection{Event-based Synchronization}

One practical synchronizing solution can be synchronizing the aggregated data based on intentionally generated sensor events. The BSN user performs some synchronization action that applies stimuli simultaneously to multiple sensors. An operator can manually 
note the times of the sensor events during post-processing. [19] describes the use of "spotting" algorithms for automatic detection of these events in multimodal sensor environments, and advocates repeating the synchronization action multiple times to avoid errors from false detections.

Event-based synchronization can be useful not only for synchronization within a BSN, but between BSNs that are not necessarily programmed to interoperate. Furthermore, it can be used to synchronize against a non-BSN system, such as an optical motion capture system for ground-truth validation of an inertial BSN experiment, but manual spotting of the events may be necessary. For example, in this study, synchronization between the TEMPO and Vicon ${ }^{\circledR}$ systems was achieved by striking a TEMPO node against a Vicon ${ }^{\circledR}$ system force plate. Similarly, video recordings of experiments can be synchronized to BSN data by selecting the video frame during which a synchronization action occurred.

\subsubsection{Time Transmission Based Synchronization}

An alternative synchronization approach utilizes the time transmission protocol (TTP) commonly used in distributed systems [20] and which has also been applied to wireless sensor networks [21]. This method allows a microcontroller to control synchronization while bypassing the inaccessible radio MAC layer in some commercial radios. In TEMPO 3.1, a star topology with a data aggregator in the center is used, i.e., communication only occurs between the aggregator and nodes, thus the TTP scheme aims to discover a relationship between the aggregator clock and each sensor node clock. When synchronizing, first clock information is exchanged between the data aggregator and the sensor nodes. Then the sensor nodes send a series of timestamps, each at time $\mathrm{T}$ 
of the nodes clock. The aggregator receives such timestamp at time $\mathrm{R}$ of the aggregators' clock, with an unknown delay d, due to the transmission delay over the radio [20]. The aggregator estimates the nodes clock based on Equation (9) [20]:

$$
T_{\text {node }}=R_{A_{n}}-\overline{R_{A}}(n)+\overline{T_{\text {node }}}(n)+\bar{d}
$$

where RA is the average of all the aggregator timestamps of received messages, RAn is the last aggregator timestamp, Tnode is the average of the node timestamp for transmitting a message, and $\mathrm{d}$ is the average delay.

This equation assumes that the clocks of both peers operate at the same basic frequency. However, in our case, this is not true - specifically, the node and aggregator maintain software timers operating at $120 \mathrm{~Hz}$ and $1 \mathrm{kHz}$, respectively - and thus we must normalize the aggregator's timestamps to those of the node by multiplying by a ratio of their basic frequencies, leading to equation (10).

$$
T_{\text {node }}=\frac{f_{\text {node }}}{f_{A}} R_{A_{n}}-\frac{f_{\text {node }}}{f_{A}} \overline{R_{A}}(n)+\overline{T_{\text {node }}}(n)+\frac{f_{\text {node }}}{f_{A}} \bar{d}
$$

Since the clock rate of each sensor node is assumed to be accurate in this paper, only the time lag (i.e., asynchronization) between multiple nodes is considered. Once these clock relationships are established, the nodes begin taking data and transmit the timestamps corresponding to when they started collecting data. The aggregator can therefore synchronize the multiple data streams based on the clock information and timestamps.

Ideally, TTP synchronization would provide the maxi- mum cross-correlation at zero. However, in reality, a deviation between the two synchronization methods can be as high 
as a few samples or tens of milliseconds. Compared to event-based synchronization, TTP synchronization eliminates a pre-defined synchronization procedure and post processing. With the cost of a small amount of delay in the beginning of data collection, TTP synchronization enables the on-body data collection to be sped up, slowed down, and even stopped and restarted without requiring the user to perform any re-synchronization.

\subsection{Orientation Tracking}

\subsubsection{Related Work}

Accurate orientation tracking using inertial BSNs have been the research focus in the field. Seemingly an intuitive product to obtain from inertial sensors, orientation information (i.e. spatial information) is not easy to extract accurately. Several reasons are associated with this: 1) signals sensed by inertial sensors are acceleration and angular velocity defined in inertial frame. In other words, inertial sensors are oblivious to the global frame. Therefore, information defined in global frame is not same as the signals inertial sensors sensed. In practice, when mounting inertial sensors on human body, it is common to have the inertial frame and the global frame misaligned, causing mounting error when global information is required. 2) Integration drift (e.g., acceleration to velocity and position, rotational rate to angular displacement). Signal processing techniques such as high-pass filtering [72], complementary filtering [28], and Kalman filtering $[23][24][25]$ have been practiced for removing the drift. 
Kalman filtering has been the mostly used techniques for drift cancellation and compensation in order to provide the accurate spatial information. This approach characterizes the noise metric in accelerometer and gyroscope, and updates the integration process accordingly given the state of parameters estimated and measured. The variations of Kalman filters are extended Kalman filter (EKF) [23][25] and unscented Kalman fitler (UKF) [26][27], which all have accurately estimated spatial information over a period of time. However, Kalman filter based approaches usually requires careful parameterization (i.e. noise characterization) before the filtering process and sample by sample matrix updating procedure, rendering the method more difficult to implement and more computationally intensive when updating on the resources constrained embedded platforms.

[28] has developed a systematic approach for extracting kinematic information by characterizing the error propagation for the Orient-2 system. In [28], complimentary filter is implemented in real-time and showing accurate results compared to extended Kalman filter, which is usually computationally intensive for embedded systems. Compared to Orient-2, the lack of magnetometer in TEMO 3.1 system may decrease its accuracy under same filter design. However, complimentary filter method will be borrowed because it is possible to implement this filter in TEMPO 3.1 system. Nevertheless, the kinematic information extracted should be further investigated for other gait feature extraction for medical applications. 


\subsubsection{Joint Angle Tracking}

In the static period, the accelerometers are employed to provide the inclination of each segment. In this section, the knee joint angle is used as an example to explain the methodology while it can be easily scaled to other orientation tracking examples. The knee joint angle is obtained by comparing the tilt of the shank and the tilt of the thigh. Following the convention of the Vicon ${ }^{\circledR}$ system, we chose the ground as the reference and subtract the thigh tilt from the shank tilt. The placement of TEMPO nodes is shown in Figure 8.

$$
\varphi_{\text {segment }}=\arctan \left(\frac{A_{x}}{\sqrt{A_{y}^{2}+A_{z}^{2}}}\right)
$$

In Equation (11), $A_{x}, A_{y}, A_{z}$ are the linear accelerations obtained from the accelerometer signals with respect to each axis, and $\varphi_{\text {segment }}$ (i.e. $\varphi_{\text {shank }}$ or $\varphi_{\text {thigh }}$ ) is defined as the angle of the $\mathrm{X}$-axis relative to ground. The knee joint angle can be found as,

$$
\varphi_{\text {knee }}=\varphi_{\text {shank }}-\varphi_{\text {thigh }}
$$

The absolute joint angle is the supplement of the angle obtained by Equation (12). We obtain dynamic gait period angles by integrating the discrete angular velocity value measured from the rate gyroscope sensors.

$$
\varphi[n]=\varphi[n-1]+\frac{\Delta}{2} \times(\omega[n-1]+\omega[n]
$$


In Equation (13), $\omega$ is the angular velocity obtained from the rate gyroscope signal, $\Delta$ is the sampling period $(1 / 128 \mathrm{~s}$ in this case) and $\varphi$ is the dynamic angle of each segment with respect to ground. The initial value of the dynamic angle will be obtained from the static posture in every experiment session providing the initial orientation for the gyroscope. The knee joint angle is calculated by subtracting the integrated thigh angle from the integrated shank angle, as shown in Equation (12).

\subsubsection{Mounting Calibration}

[22] has characterized mounting error as a dominant source of error in inertial BSNs when applied to measuring knee joint angles. In most of the applications involving spatial information computation, it can cause the data to be interpreted with a reduced angular velocity, thereby affecting the accuracy of spatial information extraction. Mounting error exists when the sensor is not affixed to the body in the assumed orientation. Ideally the sensor coordinate frame should align with the body frame as shown in Figure 10.

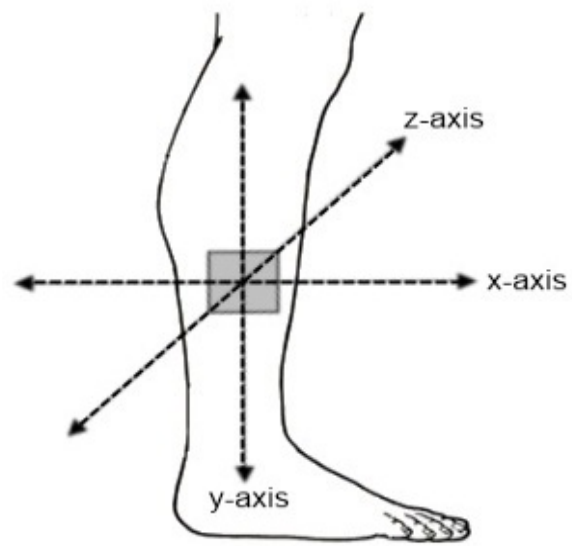

(a) Ideal mounting

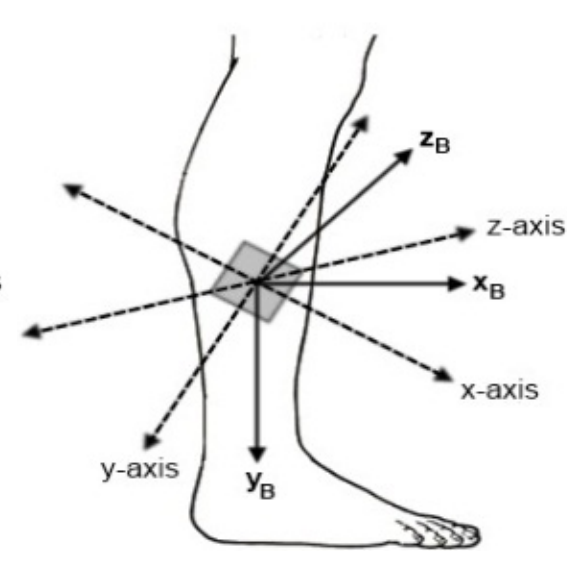

(b) Non-ideal Mounting

\section{Figure 10 An Inertial BSN Node and Corresponding Axis Orientation}


In order to correct the error due to imperfect mounting, the orientation of the inertial frame (sensor coordinate frame) with respect to the body frame needs to be determined. To achieve this, two vectors are computed and then used to relate the two frames. These vectors, $\boldsymbol{y}_{\boldsymbol{B}}$ and $\boldsymbol{z}_{\boldsymbol{B}}$, are the $\mathrm{y}$ and $\mathrm{z}$ axes of the body frame represented by the coordinates of the inertial frame and can be determined by the pre-defined mounting calibration procedure detailed in the rest of this section. $\boldsymbol{x}_{\boldsymbol{B}}$, the x-axis of the body frame, is determined as the cross product of $\boldsymbol{y}_{\boldsymbol{B}}$ and $\boldsymbol{z}_{\boldsymbol{B}}$.

To deterimine $\boldsymbol{y}_{\boldsymbol{B}}$, the mounting calibration procedure requires the subject to stand straight with her/his leg vertical to ground. Thus the y-axis of body frame, which is also the direction of gravity in this posture can be represented in the inertial frame as:

$$
\boldsymbol{y}_{\boldsymbol{B}}=\left[\begin{array}{lll}
a_{x} & a_{y} & a_{z}
\end{array}\right]^{T}
$$

$a_{x}, a_{y}$, and $a_{z}$ represent the readings from the $\mathrm{x}, \mathrm{y}$, and $\mathrm{z}$ axes of accelerometer respectively. This representation is based on the assumption that during standing straight posture, the gravity vector in the inertial frame is parallel to $\boldsymbol{y}_{\boldsymbol{B}}$.

To determine the z-axis of the body frame in the inertial frame, it is necessary to rotate about the body frame's z-axis and find the axis of rotation in the inertial frame. In this mounting calibration procedure, the subject should lift his/her leg forward in the sagittal plane and hold the leg steady by resting it on a surface in order to isolating the gravitation force vector by minimizing the linear motion The reading from the accelerometers at this new position is recorded as:

$$
\boldsymbol{g}^{\prime}=\left[\begin{array}{lll}
a_{x}^{\prime} & a_{y}^{\prime} & a_{z}^{\prime}
\end{array}\right]^{T}
$$

$\boldsymbol{z}_{\boldsymbol{B}}$ is then determined as: 


$$
z_{B}=y_{B} \times g^{\prime}
$$

The principle behind this procedure is that $\mathbf{z}_{\boldsymbol{B}}$, the axis around which the gravity vector is rotated, is perpendicular to both $\boldsymbol{y}_{\boldsymbol{B}}$ and $\boldsymbol{g}^{\prime}$ and thus can be represented by their cross product. The greater the rotation of the gravity vector (i.e. the higher the leg is lifted), the more accurate this calibration will be. In addition, because $\boldsymbol{z}_{\boldsymbol{B}}$ and $\boldsymbol{g}^{\prime}$ are only orthogonal when the leg is lifted 90 degrees, it is necessary to normalize the vector $\boldsymbol{Z}_{\boldsymbol{B}}$. To determine $x_{B}:$

$$
x_{B}=y_{B} \times z_{B}
$$

With these three column vectors, $\boldsymbol{x}_{\boldsymbol{B}}, \boldsymbol{y}_{\boldsymbol{B}}$, and $\boldsymbol{z}_{\boldsymbol{B}}$, it is possible to construct a rotation matrix to transform vectors in the inertial frame to their corresponding vectors in the body frame. This matrix is defined as:

$$
R^{B I}=\left[\begin{array}{lll}
x_{B} & y_{B} & z_{B}
\end{array}\right]
$$

The two measurements used for determining gait speed are the measurements from the triaxial accelerometer and the measurements from the tri-axial gyroscope. Both of these are taken in the reference frame of the sensor but need to be viewed from the reference frame of the body. In the inertial frame, the acceleration vector and the angular velocity vector are respectively represented:

$$
\begin{aligned}
\mathbf{a} & =\left[\begin{array}{lll}
a_{x} & a_{y} & a_{z}
\end{array}\right]^{T} \\
\boldsymbol{\omega} & =\left[\begin{array}{lll}
\omega_{x} & \omega_{y} & \omega_{z}
\end{array}\right]^{T}
\end{aligned}
$$

The acceleration and angular velocity vectors in the body frame, $\mathbf{a}_{\mathbf{B}}$ and $\boldsymbol{\omega}_{\mathbf{B}}$, are transformed by $\boldsymbol{R}^{\boldsymbol{B I}}$ : 


$$
\begin{gathered}
\mathrm{a}_{\mathrm{B}}=R^{B I} \mathrm{a} \\
\omega_{\mathrm{B}}=R^{B I} \omega
\end{gathered}
$$

Therefore, by transforming all of the measurements taken in the inertial frame to measurements in the body frame, the mounting error is minimized.
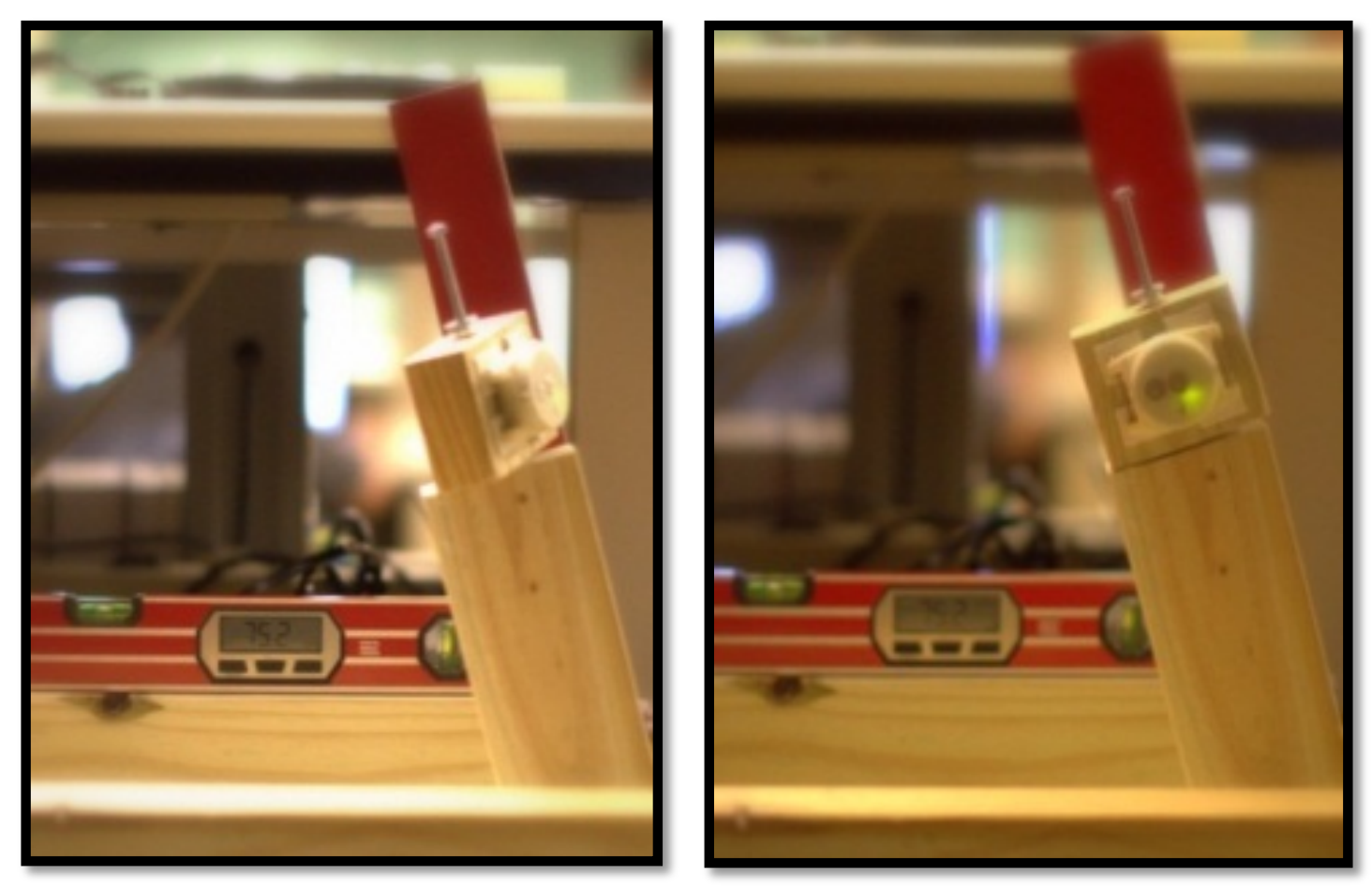

Figure 11 Validation of Mounting Calibration Method

To verify the mounting calibration algorithm, a pendulum model shown in Figure 11 was employed. A sensor node was affixed to the pendulum only rotating around the z-axis. The node was mounted with different controlled degrees of error around the y-axis. The angle rotated around the y-axis was then extracted from the rotation matrix produced by the algorithm. The results of the error minimized are shown in Table 1. 
Table 1 shows the effectiveness of the mounting calibration algorithm in measuring the mounting error. The average error between the angle measured using an inclinometer and the angle measured by the algorithm was $1.023^{\circ}$. Given the margin error of the inclinometer measurement, the results show that this method can accurately determine the spatial orientation of the sensor node with respect to the body frame.

Table 1. Angles measured at various mounting errors.

\begin{tabular}{|c|c|c|}
\hline Mounting Position & Measured by & Measurement \\
Rotated Around Y-axis & Current Method & Error of Angle \\
\hline $0^{\circ}$ & $-0.072^{\circ}$ & $0.072^{\circ}$ \\
\hline $15^{\circ}$ & $16.286^{\circ}$ & $1.286^{\circ}$ \\
\hline $30^{\circ}$ & $27.896^{\circ}$ & $2.104^{\circ}$ \\
\hline $45^{\circ}$ & $43.954^{\circ}$ & $1.046^{\circ}$ \\
\hline $60^{\circ}$ & $58.078^{\circ}$ & $1.922^{\circ}$ \\
\hline $75^{\circ}$ & $74.737^{\circ}$ & $0.263^{\circ}$ \\
\hline $90^{\circ}$ & $90.461^{\circ}$ & $0.461^{\circ}$ \\
\hline
\end{tabular}

\subsubsection{Filtering Techniques}

Integration drift is inherent in any inertial sensor system and occurs when converting from acceleration to velocity (or again to position displacement) or from rotational rate to angular displacement. Here we focus on the latter, as discrete integration is applied to the gyroscope data in Equation (13). It is well-known that drift occurs as a function of time 
and acts as a DC offset in the gyroscope due to intrinsic sensor errors such as random noise and bias that are accumulated with time during integration, this DC offset can be effectively removed with a high-pass filter, as was done for the results in the other sections.

To do so, a sensor node was placed on the turntable from 1 full rotation to 10 rotations with an increment of 1 rotation, and for 15,20 , and 30 rotations, with a time span of $\sim 1.8$ $\mathrm{s}$ at $200 \% \mathrm{~s}$ and $\sim 1.33 \mathrm{~s}$ at $270 \% \mathrm{~s}$ for each rotation. The experiment for each rotation number was repeated three times to reduce the uncertainty and observation error, with a standard deviation no larger than $5^{\circ}$. The experiments were performed in both the positive and negative directions. The integration error is computed as the deviation of the integrated angles using the raw angular velocity (calibrated using the above piecewise method) from the reference angles (i.e., number of rotations $\times 360^{\circ}$ ). The results are shown in Figure 12. 


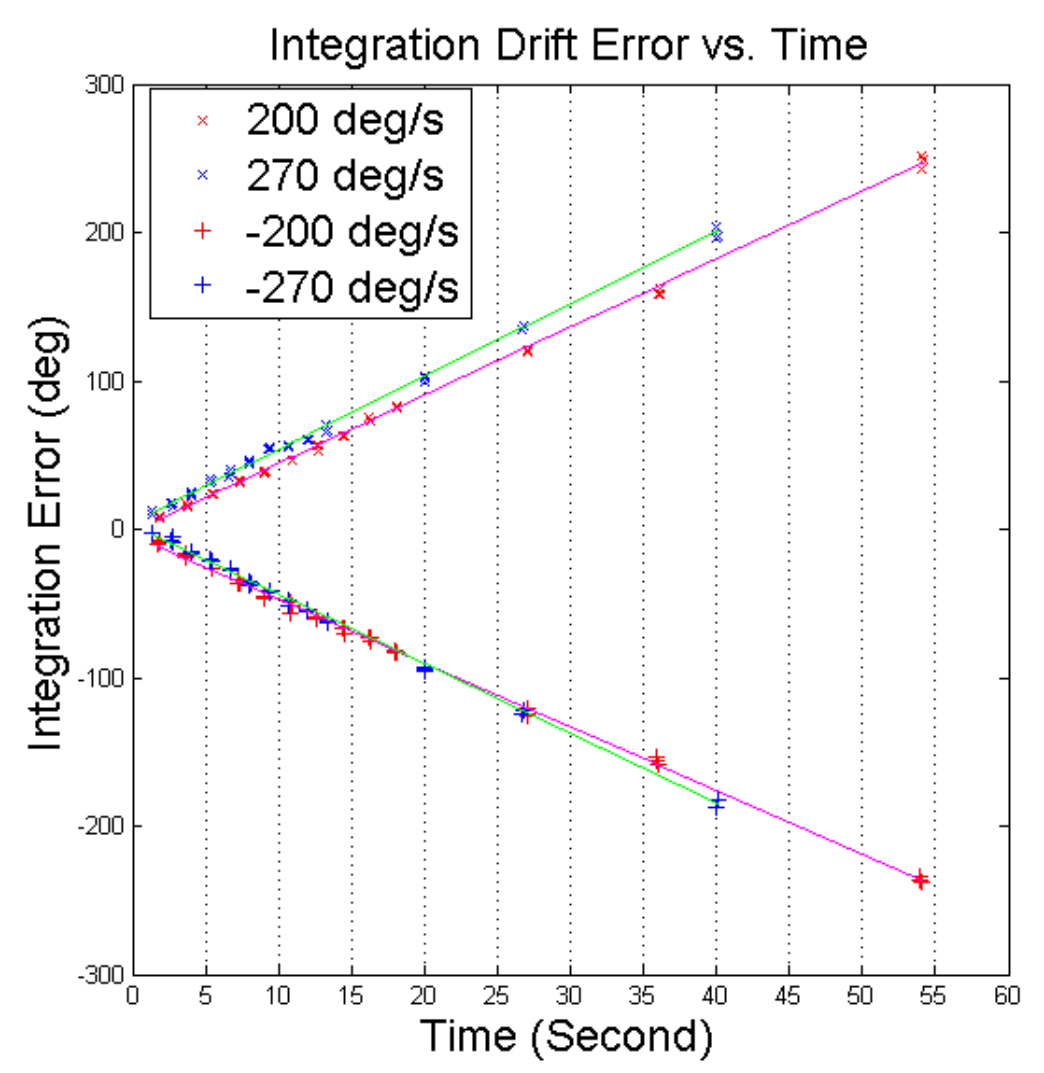

Figure 12 Gyroscope Integration Drift

To replicate the method used in [28], in this dissertation, a $3^{\text {rd }}$ order Butterworth highpass filter with a cut-off frequency of $0.3 \mathrm{~Hz}$ is applied. To avoid phase-distortion caused by filtering, the filter is applied twice, forward and backward, to achieve zero-phase distortion. A high-pass filter inevitably removes the DC signal. To compensate for this, complimentary filter can be applied to further utilize accelerometer reference.

For the gyroscope signal, a high pass filter with a cut-off frequency of $0.3 \mathrm{~Hz}$ is designed, and for the accelerometer signal, a complementary low-pass filter is designed at the same cut-off frequency. To minimize the overshooting effect, a $1^{\text {st }}$ order Butterworth filter is 
selected. The principle is to filter out the offset of the gyroscope signal while maintaining the local motion. For the accelerometer whose orientation is reliable in the long-term, the higher frequency motion is filtered out. To avoid phase-distortion caused by filtering, both the high-pass and low-pass filters are applied twice, forward and backward, to achieve zero-phase distortion.

After low-pass filtering, the resultant acceleration is almost entirely due to gravity, which varies with the sensor orientation and can be determined by Equation (11). Overall, complementary filter design is equivalent to Equation (23) in the time domain:

$$
S=0.98 \times \text { Angle }_{\omega}+0.02 \times \text { Angle }_{A}
$$

where Angle $\omega_{\omega}$ represents the gyroscope signal informed angle via Equation (13) and Angle $_{A}$ represents the accelerometer signal informed angle information via Equation (11). In summary, the target signal $S$ contains orientation information and can be considered a weighting function of the angles obtained from both the gyroscope and accelerometer signals.

\subsection{Summary}

In this chapter, time synchronization, sensor calibration, integration drift, and mounting calibration were detailed to minimize sources of errors in order to accurately extract kinematic information from inertial BSNs. Since such errors are inherent in any multinodal inertial BSN system and can greatly impact the fidelity of kinematic information, it is necessary to characterize such errors within the target application. Whilst 
synchronization errors and calibration errors can be minimized significantly, mounting error is proved to be the biggest challenge when the nodes are placed on human body in practice - dominating the error impact on information fidelity. Compensation methods for mounting error were explored that provided accuracy up to an RMSE of $\sim 1.5$ degrees during walking, which is within the range of error in the optical motion capture system. Meanwhile, the integration drift presents challenge in accurately extracting spatial information. With a designed complimentary filter, this error can also be minimized to suffice the application requirement. This method will be validated in Chapter 6 via a case study. 


\section{Chapter 3}

\section{Linear Analysis for Temporal Feature Extraction}

Chapter 2 details methods of transforming raw inertial BSN data into the primary product - kinematic information, which preserves the time series nature of the signal at a high sampling rate. To relate such information to medical outcome, more in-depth information needs to be extracted from the kinematic information. As human gait is a repetitive motion, time series of gait signals possess pseudo-periodic nature. This means that repetitive events in a cycle can be detected and extracted to examine the temporal features of the gait. In this chapter, methods of detecting critical gait events are presented based on the observation of human gait. These features often tend to employ linear analysis for 1-D time series, hence called linear features.

\footnotetext{
This chapter adapts from publication [P9]
} 


\subsection{Gait Specific Temporal Features}

Gait specific temporal features are the characteristic features that only exist in human gait [29] [34]. Extracting and analyzing such features help to segment gait motion in time. In this section, the following features will be explored and extracted: Gait Cycle Segmentation, Cadence, Stance Phase, Swing Time, Single Support Time, Double Stance Time, Stride Length, and Gait Speed.

\subsubsection{Gait Cycle Segmentation and Cadence}

Gait cycle extraction [37] is critical to extract parameters such as gait phase, stride time (i.e. inverse of cadence), and stride length. Examining gyroscope signal of the shank mounted inertial sensor, we find that the periodical nature of the signal can be utilized perfectly for gait cycle segmentation with peak detection algorithm. To avoid the detection algorithm stuck in the local maximum within a small range, the ripples in the gyroscope signal are suppressed using a zero-phase, 3rd order, Butterworth low-pass filter with a cutoff frequency of $3 \mathrm{~Hz}$. The cutoff frequency is determined empirically by inspecting the spectrum of the gyroscope signal, in which the main frequency components lie below $3 \mathrm{~Hz}$. With the pronounced peaks, a local maximum peak detection algorithm can be used to identify the maximum of the sinusoidal-like signal as the green dots shown in Figure 13. 


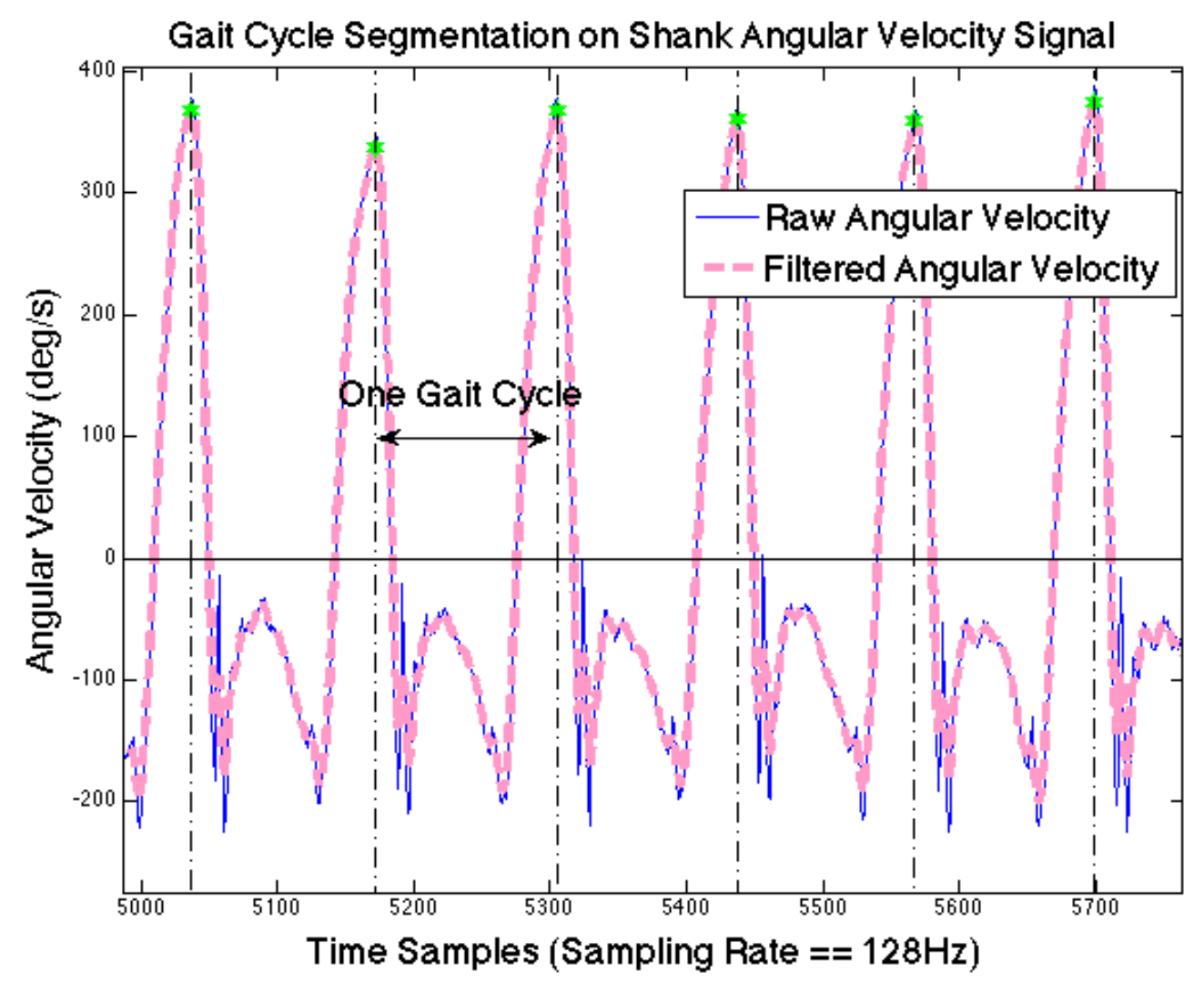

Figure 13 Gait Cycle Segmentation

After accurately separating gait cycles, stride time extraction becomes a trivial problem. With high sampling rate, this temporal information can be accurately obtained by counting the samples inside one gait cycle. The stride time will be the number of samples divided by sampling frequency. Cadence is a product by taking the inverse of the stride time.

\subsubsection{Gait Events}

To better study the human walking mechanism, photos are taken on the street of many pedestrians walking. At a rate of 8 frames/second, a continuous motion as walking that can not be decomposed by human eyes are captured clearly and segmented by a series of 
static pictures, as shown in Figure 14. Confirming that all common pedestrians are walking in the same fashion by checking different pedestrians' walking photos, we abstract the photos into Figure 15 .
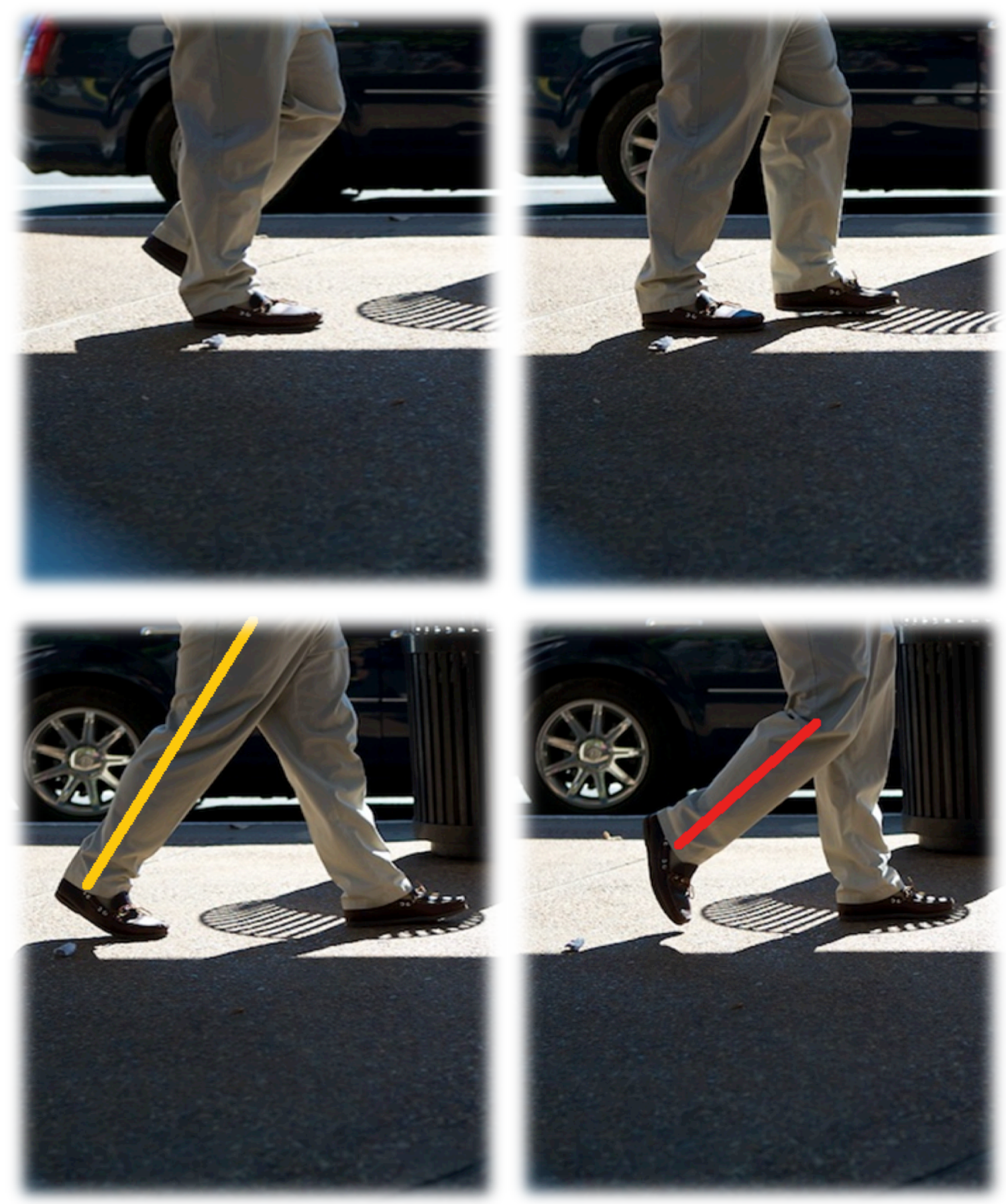

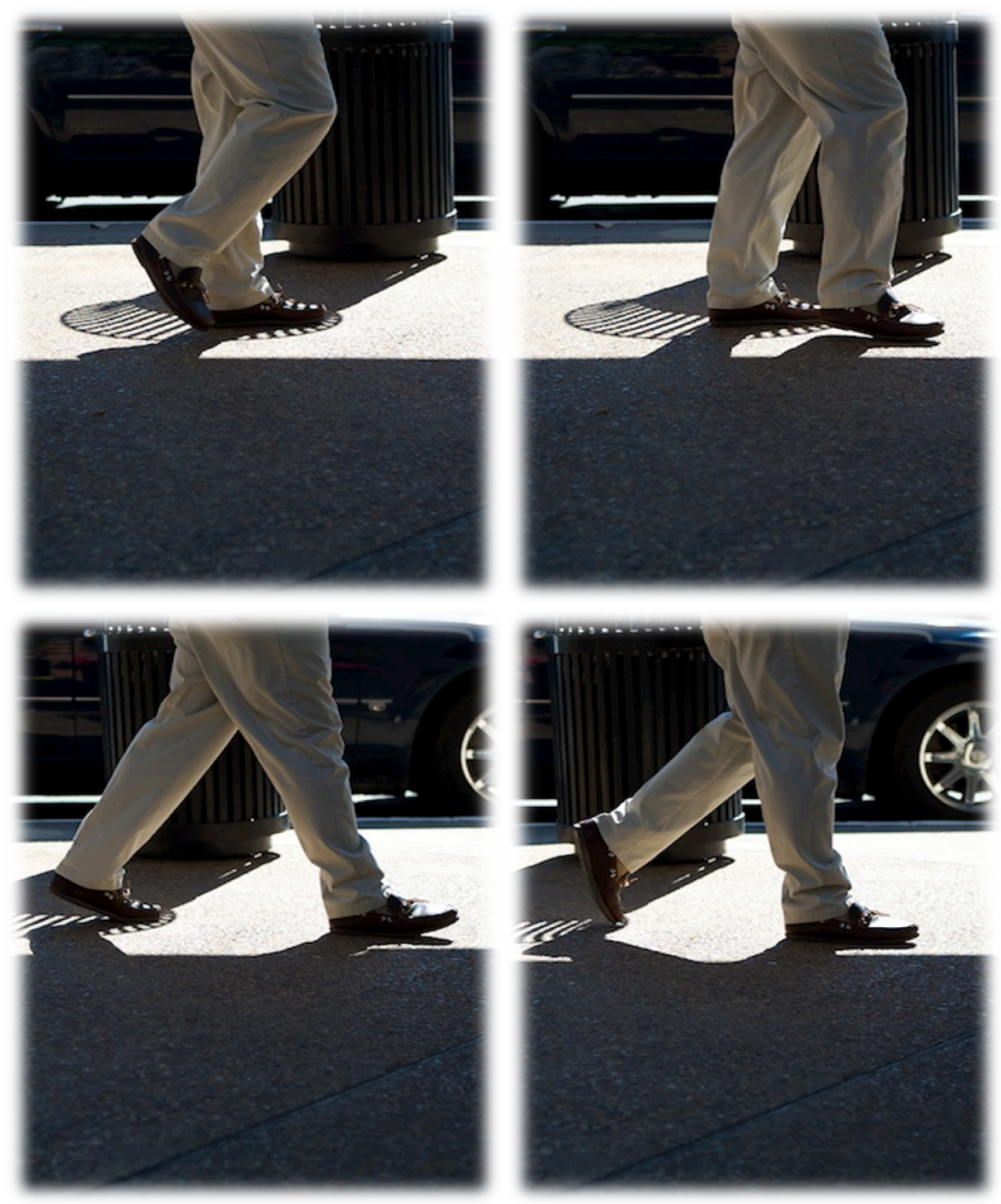

Figure 148 Frame/Second Shots of Gait Events in One Gait Cycle 


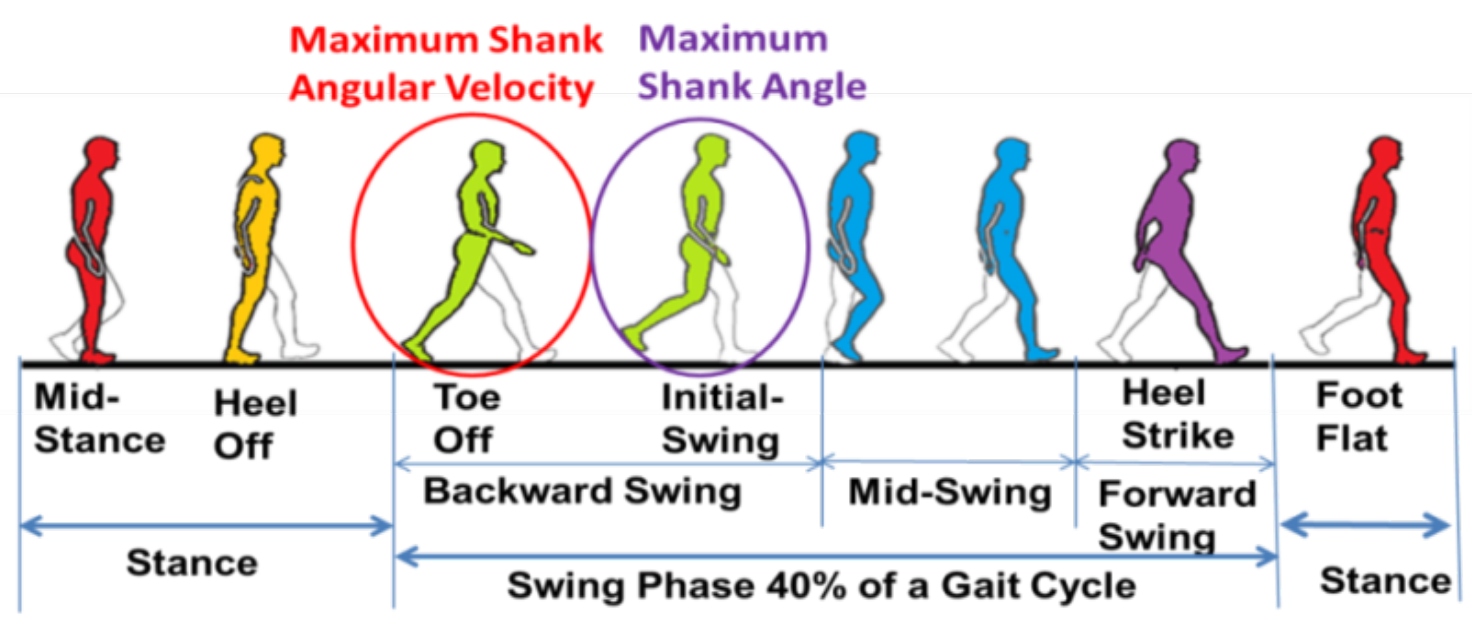

Figure 15 Gait Events During One Gait Cycle

Figure 14 and Figure 15 illustrate the decomposed gait events in a gait cycle, universally. Taking the right leg on both figures for example, during walking, there is always a moment that a human subject with right leg standing on the ground (Mid-Stance event). Starting from this moment, in order to move forward, the subject lifts her/his heel off, pushes backwards on the ground (Heel Off event) in order to give the body a counterforce to lean forward until she/he can completely lift her/his toe in the air (Toe Off event). The right leg continues to swing backward in order for the shank to reach the highest potential (Initial Swing event) as a pendulum. Then after reaching the highest potential, the right leg in the air swings forward while transforming the potential energy to kinetic energy, without extra effort (Mid-Swing phase). When the right leg reaches the lowest point, the right foot hits the ground (Heel-Strike event), lands and supports the body weight (Foot-Flat Event), and waits for the other leg to swing (Mid-Stance event as the beginning of the next cycle). This cyclic motion can be found for the left leg, as the two legs alternate. 
Figure 13 provides a good intuition of detecting the gait events in the time domain. With the gait cycle segmented, we can identify the different events by the occurring sequence.

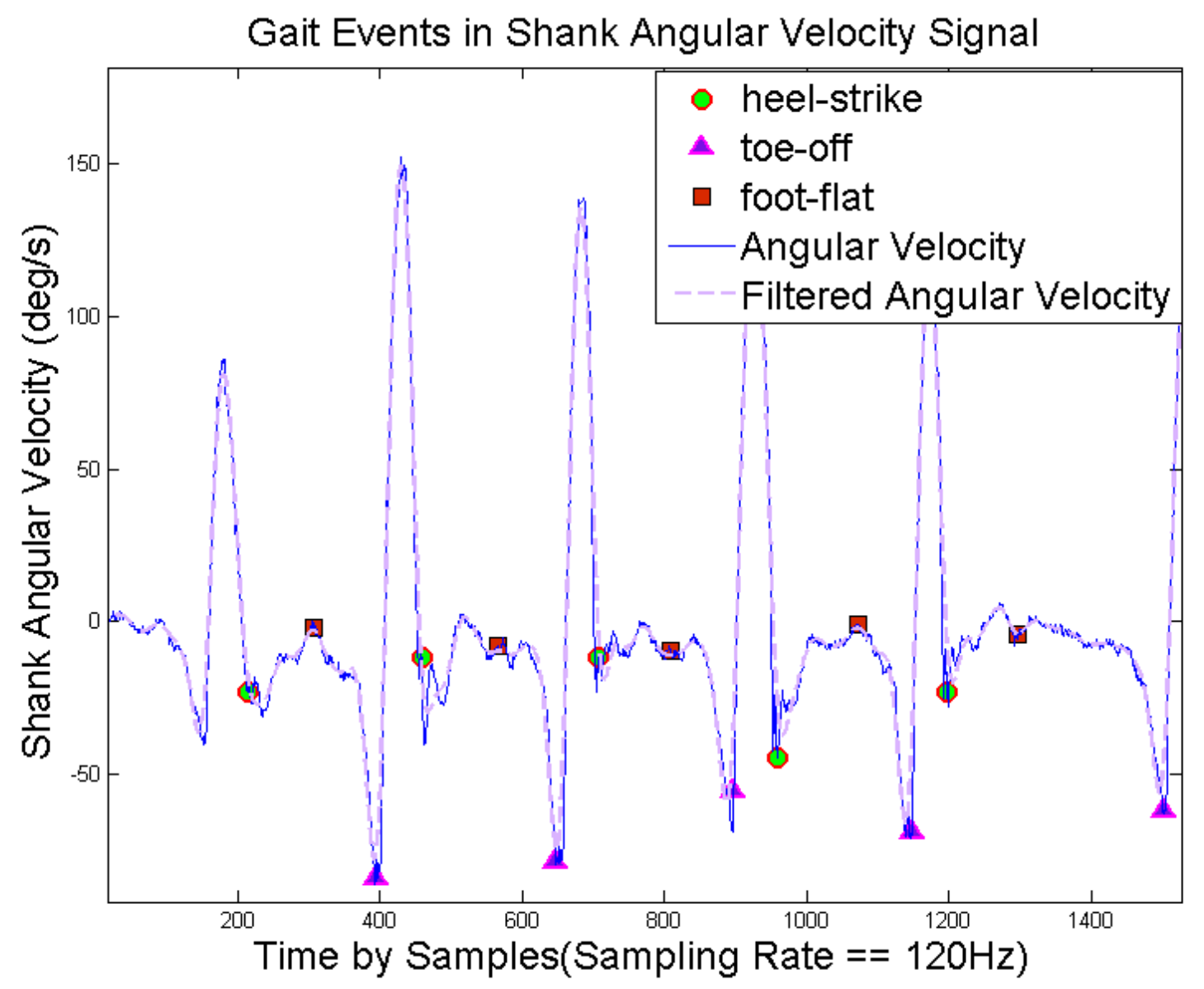

Figure 16 Critical Gait Events Detection on Gyroscope Signal

\subsubsection{Gait Temporal Features}

With the gait events detected successfully, we can extract temporal features such as double stance time, swing time etc., based on the timestamps of the events. However, the critical gait phases must be learned first in the context of the gait events. Figure 17 
defines these temporal features by incorporating the gait events and phases, providing a graph to search for the temporal features depicted in the following sections.

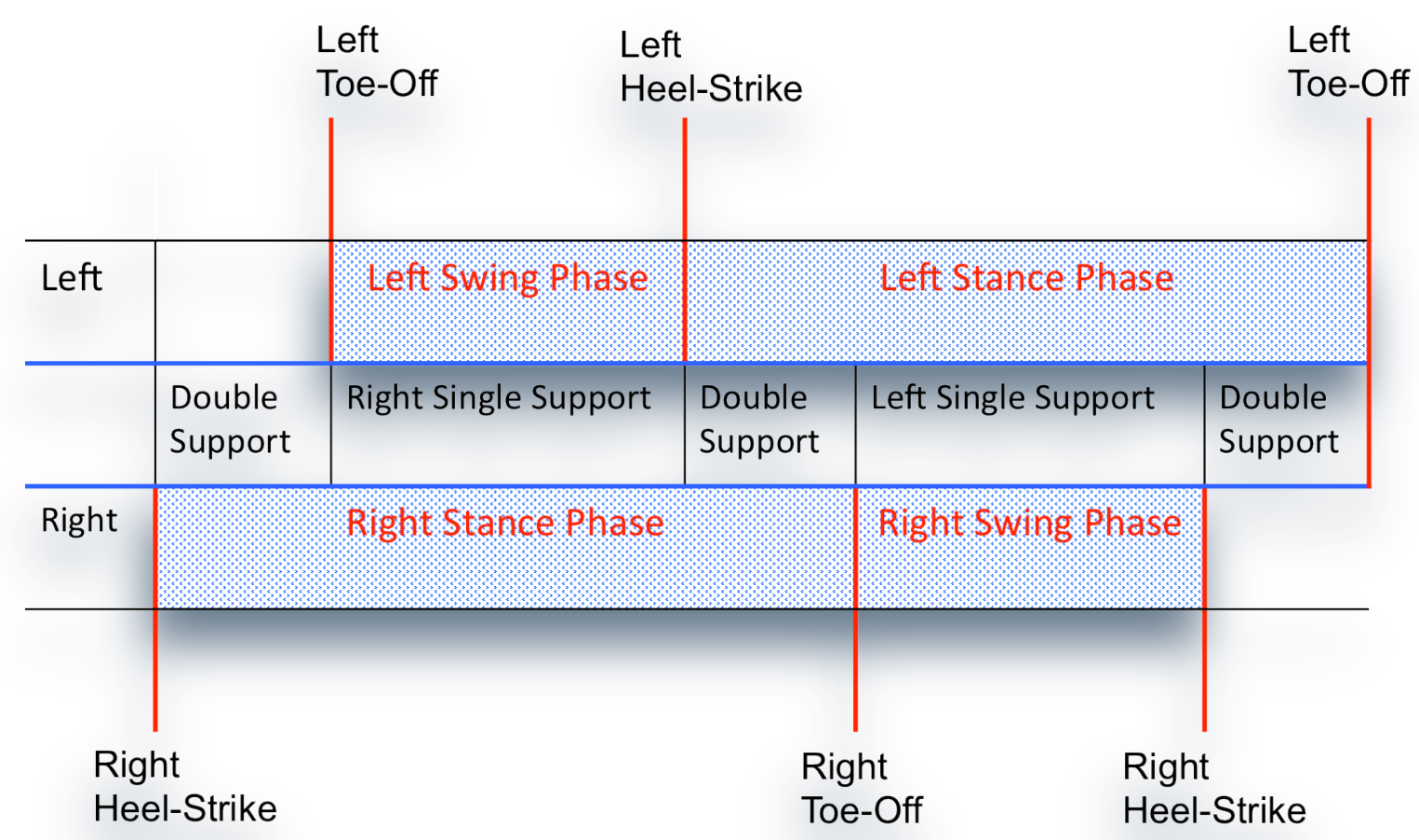

Figure 17 Gait Phase Explained

\subsubsection{Swing Time (SWT)}

Figure 17 shows that swing time is the duration between Toe-Off gait event and HeelStrike gait event of one leg inside one gait cycle. During this phase, the leg first pushes backward and then swings forward, transforming the potential energy into kinetic energy, and resulting the highest value in acceleration signal and angular velocity signal. To find the duration, a sorting algorithm can be used to label the two sequential, adjacent Toe-Off 
and Heel-Strike events, and count the number of samples between these two timestamps and get single support time. Note this feature only relies on one leg's inertial sensor data.

\subsubsection{Single Support Time (SST)}

Figure 17 shows that single support time is the duration between Toe-Off gait event and Heel-Strike gait event and of one leg inside one gait cycle. During this phase, the foot lands on the ground and the leg gradually rotates centered around the foot until the center of mass of the whole body moves forward. Interestingly, one leg's single support time is exactly the same as the swing time of the other leg. Note this feature only relies on one leg's inertial sensor data.

\subsubsection{Stance Phase (SP)}

Figure 17 shows that stance phase time is the duration between Heel-Strike gait event and Toe-Off gait event of one leg inside one gait cycle. During this phase, the foot lands on the ground and the leg gradually rotates centered around the foot until the center of mass of the whole body moves forward. To find the duration, a sorting algorithm can be used to label the two sequential, adjacent Heel-Strike and Toe-Off events, and count the number of samples between these two timestamps and get single support time. The difference between single support time and stance phase is that the latter includes the double support time where both feet are on ground. Note this feature only relies on one leg's inertial sensor data. 


\subsubsection{Double Stance Time (DST)}

Double stance time is the phase of both feet in contact with the ground during walking. Figure 17 shows that double stance time is the duration between Heel-Strike event of one leg and the Toe-Off event of the other leg. As an information involving coordination from both legs, it is tricky to accurately obtain since its accuracy depends on timestamps from both legs instead of one. Here, the synchronization between the nodes becomes critical to this problem. However, with a careful examination of Figure 17, this feature can be extrapolated as:

$$
D S T=S P-S W T
$$

\subsection{Stride Length and Gait Speed}

Gait speed is an important parameter in gait analysis. In geriatrics specially, gait speed has become the number one predictor of mortality in adults over 65 years old, with differences of just a couple tenths of a meter per second predicting statistically significant outcome differences [35]. Thus accurate gait speed estimation from inertial BSNs has interested researchers in the field [36] [45] [58][59][60].

\subsubsection{Related Work}

Early, previous work using inertial sensors to estimate gait speed usually modeled human gait as an inverse pendulum [36] [42] [58] [59]. [36] was the first to devise the method of using a single axis gyroscope to estimate stride length and gait speed, with a single pendulum model. The method intuitively explained how to use geometric model to 
extract gait speed from inertial sensors and achieved an accuracy with relative errors of $15 \% \sim 25 \%$ over a speed range of $0.5 \mathrm{~m} / \mathrm{s} \sim 1.7 \mathrm{~m} / \mathrm{s}$. [58] proposed a more precise model by using both shank and thigh mounted inertial sensors with better defined geometric model, achieving an RMSE of $0.06 \mathrm{~m} / \mathrm{s}$ at a constant treadmill speed $(1.11 \mathrm{~m} / \mathrm{s})$. While the initial efforts in [36] seemed to provide an over-simplified model, the more refined model in [58] requires thigh nodes, which introduces more invasiveness to the wearer (an issue of both node location and number). In this dissertation, the invasiveness and mounting uncertainty of thigh-mounted nodes is avoided by the use of a refined gait model that only requires shank data. [42] also employed the double pendulum model, with Kalman filter to cancel drift in the gyroscope integrated signal, achieving a stride length RMSE of $0.05 \mathrm{~m}$ per stride.

[38] explored the possibility of using foot-mounted inertial sensors to obtain linear velocity from accelerometer by leveraging the gyroscope integrated angle information, achieving an RMSE across 5 subjects ranging from $0.03 \mathrm{~m} / \mathrm{s} \sim 0.06 \mathrm{~m} / \mathrm{s}$. [59] took similar approach, but instead of using mount the sensors on the foot, it used shank-mounted inertial sensors and achieved an RMSE of $0.05 \mathrm{~m} / \mathrm{s}$ from $0.8 \mathrm{~m} / \mathrm{s}$ to $1.8 \mathrm{~m} / \mathrm{s}$ with $0.2 \mathrm{~m} / \mathrm{s}$ increment. Although integrating acceleration to obtain distance and velocity seems an intuitive approach, the accuracy can be worse because gravitational force is difficult to separate from inertial force and accelerometers are susceptible to linear mechanical noise. To achieve accurate results using method described in [38] and [59], careful noise reduction and integration drift cancelation method are required, rendering the method less robust in implementation. 
[41] [43] [45] and [60] resorted to machine learning approaches for estimating gait speed. [41] adopted Gaussian process regression (a nonlinear regression approach) to estimate gait speed from frequency domain features, achieving an average RMSE of 0. $027 \mathrm{~m} / \mathrm{s}$ observed at one subject. [43] and [44] used the decomposed wavelets from accelerometer features as features, and used linear regression approach to estimated gait speed, achieving an average error below 5\%. [45] estimated gait speed as a middle product for energy expenditure estimation. With a hip-mounted acceleration tracking cadence, it achieved an average error of $0.18 \mathrm{~m} / \mathrm{s}$. These pioneer works using statistical learning methods laid groundwork for the field moving from first principle modeling to machine learning for gait speed estimation. However, the features mentioned in these work and the accuracy of the results need further validation via a unified machine learning framework. The machine learning based approach will be further discussed in Chapter 5 .

[39] reviewed the current research (16 papers in total) on gait speed estimation using inertial sensors, categorizing the current gait speed estimation model in three categories: abstraction model (i.e. machine learning approach), human gait model and direction integration. With the existing effort in the field, it is worthwhile to combine the advantages of all these approaches in a framework, as will be discussed in Chapter 5. 


\subsubsection{Stride Length Computation}

Stride length computation is the critical step of getting gait speed. With stride length being accurately extracted, gait speed can also be obtained with the combination of accurate stride time. The overall steps for computing gait speed are:

\section{1) Calculating Stride Length/Gait Cycle}

2) Distance $=\sum_{i=1}^{i=\text { No.of Cycles }}$ Stride Length of Gait Cycle i

3) Average Gait Speed = Distance / Travelling time

Discrete integration as shown in Equation (13) introduces inevitable drift as a function of time due to gyroscope sensors' bias and random noise. The gait cycle information is leveraged to null the drift based on the evidence that at the foot-on-ground events the shank angle should be near zero. Since integration can be conducted in a small duration (one gait cycle in this case, no longer than 3 seconds), it is reasonable to assume the drift is accumulated due to constant bias over random noise that is linear over time. Therefore, at the initial and end instance of a gait cycle (foot-on-ground events), the angle integrated of these two instances can be reset to zero, hence eliminating the integration drift in a gait cycle:

$$
\left\{\begin{array}{c}
\text { Slope }=\frac{\theta_{\text {end }}-\theta_{\text {start }}}{T_{\text {end }}-T_{\text {start }}} \\
\hat{\theta}_{i}=\theta_{i}-\text { Slope } \times i
\end{array}\right.
$$

where Slope is the slope of the drift assumed linearly in one gait cycle, $\theta_{\text {start }}$ and $\theta_{\text {end }}$ denote the shank angle at the start and end point respectively of the gait cycle, $T_{\text {start }}$ and $T_{\text {end }}$ denote the timestamp at the start and end point respectively of this gait cycle, $\theta_{i}$ is 
the shank angle at time index $i$ of this gait cycle, and $\hat{\theta}$ is the shank angle after drift cancellation at time index $i$ of this gait cycle, as used to find the swing range.

To quantitatively examine the different gait events occurring in one gait cycle, Figure 18 presents both angular velocity and angular position in time domain plot and labels out the gait events on the signals. After examining this figure, we found that the angular velocity of the shank reaches its maximum when the leg is fully extended, and the angle of the shank reaches its maximum after this when the leg is flexed. These two events do not overlap in time. This is further confirmed as the human gait photos captured by the 8frames/second series shown in Figure 14.

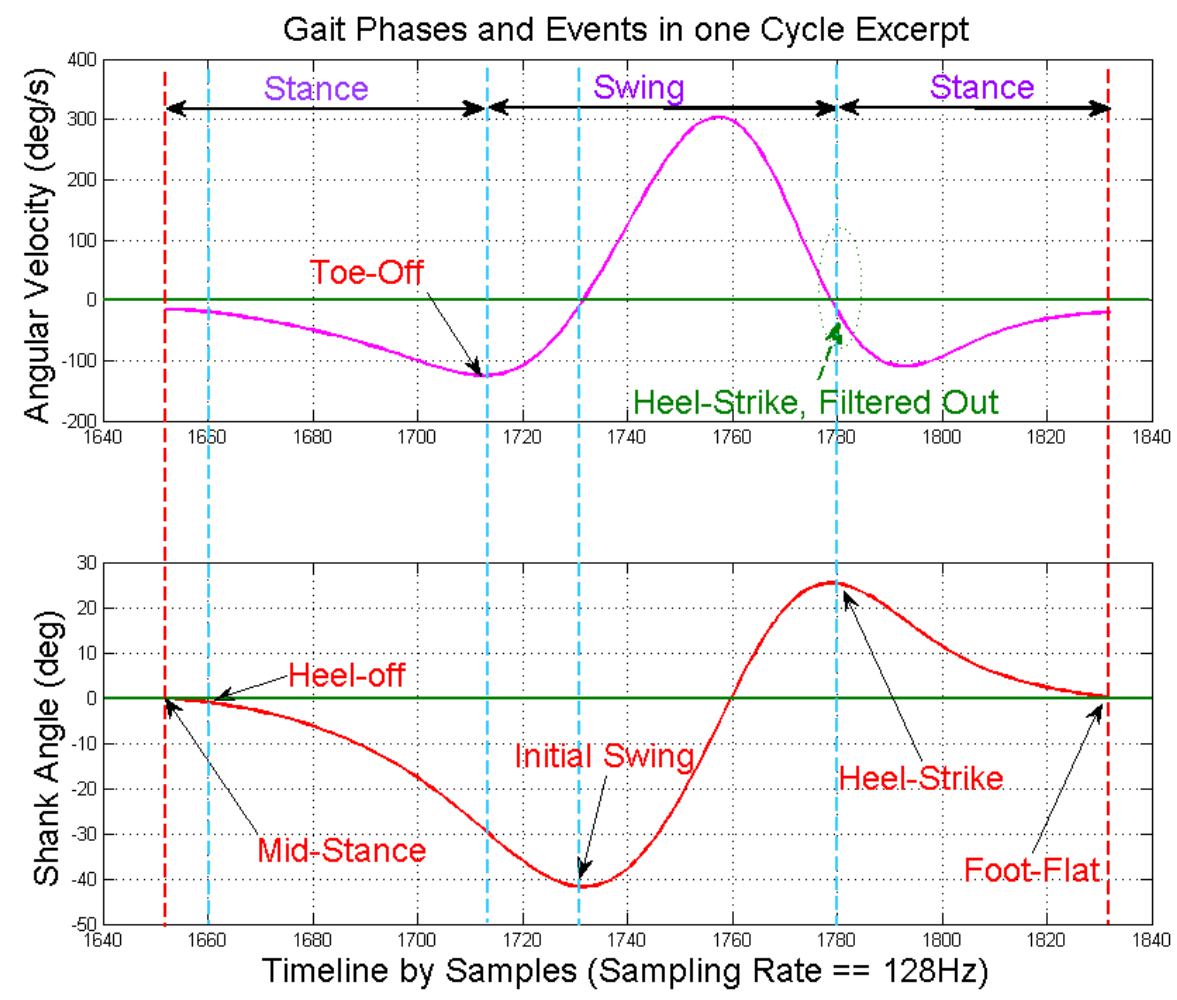

Figure 18 Gait Events in One Cycle's Time Domain Signal 


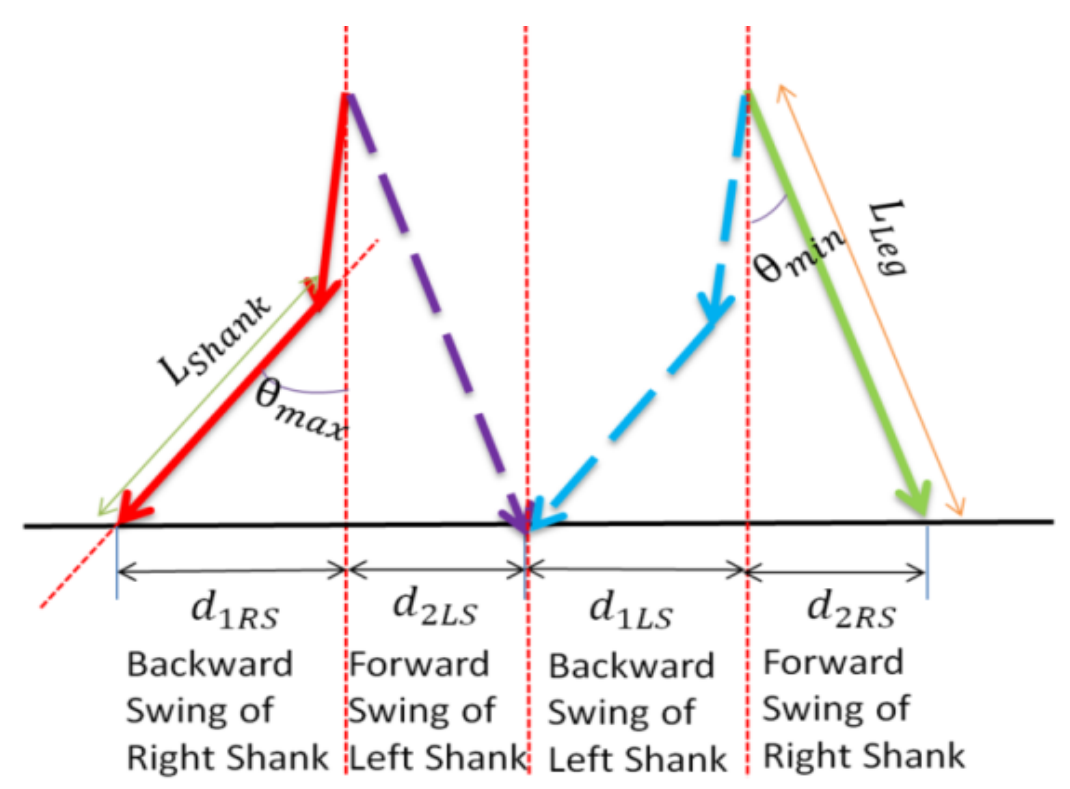

Figure 19 Double Pendulum Model of Gait

Based on the observation from Figure 14 and Figure 18, a double pendulum model is devised to extract stride length as shown in Figure 19. Since healthy human gait is relatively symmetric, the computation model can be applied to both shanks (assymetric models may have to be used for pathological gait). Here, the right shank is used as an example for computation:

$$
\begin{gathered}
d_{1 R S}=\sin \left(\theta_{\max }\right) \times L_{\text {Shank }} \\
d_{2 R S}=\sin \left(\theta_{\text {min }}\right) \times L_{\text {Leg }}
\end{gathered}
$$

The stride length of one gait cycle can be considered as:

$$
\text { Stride_Length }=d_{1 R S}+d_{2 L S}+d_{1 L S}+d_{2 R S}
$$

The shank angle range is obtained by integrating the discrete angular velocity value measured from the rate gyroscope sensors described in Equation (13). The swing range 
can then be found by differencing the maximum and minimum of the shank angle in one gait cycle.

\subsubsection{Evaluation of Gait Speed Estimation Method}

The accuracy of the estimation was verified using a treadmill. Two walking trials at $1.2 \mathrm{~m} / \mathrm{s}$ on both ground and treadmill are studied to establish the assumption that the gait pattern of the sagittal plane gyroscope signal on treadmill do not differ from it on ground. Two healthy subjects, one female and one male, walked on the treadmill mounted with inertial BSN nodes on both shanks. An elastic Veclro ${ }^{\circledR}$ strap and racquet handle grip were used to maintain mounting position. The subjects then were asked to walk on the treadmill at speeds ranging from 1 to $3 \mathrm{MPH}$ with a $0.2 \mathrm{MPH}$ increment for 45 seconds each. The data from the shank mounted sensors were recorded on a laptop and post processed in Matlab ${ }^{\circledR}$ for information retrieval and data analysis.

The method described in Section 3.2.2 is compared with the single pendulum model described in [36]. The RMSE is computed for both methods comparing treadmill speed, with a resolution of $0.2 \mathrm{MPH}(0.09 \mathrm{~m} / \mathrm{s})$ from $1 \mathrm{MPH}$ to $3 \mathrm{MPH}$, to the calculated gait speed. The accuracy of the proposed model was significantly higher than that of the reference [36], which commonly overestimates gait speed, shown in Figure 20 and Figure 21. The improvement of using double pendulum model stated in Section 3.2.2 shows that with in-depth biomechanic knowledge, the accuracy of gait feature extraction can be improved without raising the complexity of signal processing or without wearing additional inertial BSN nodes. 


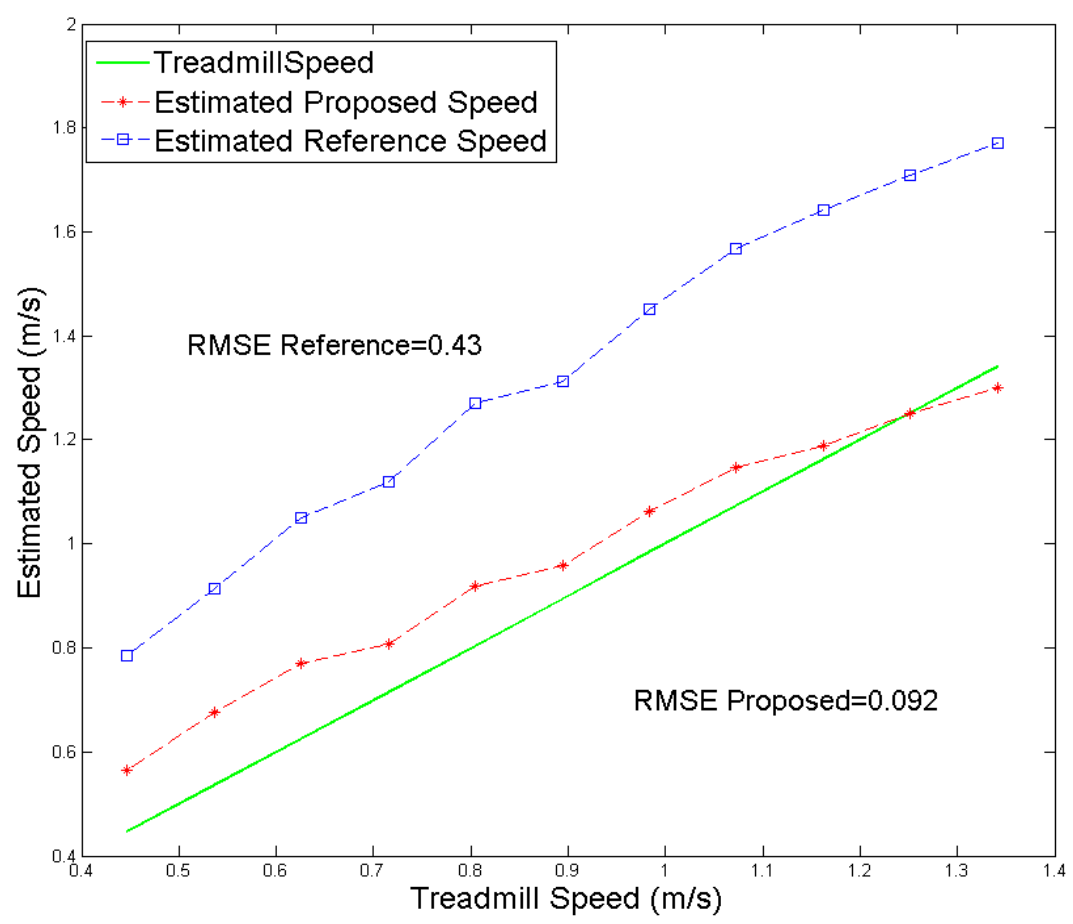

Figure 20 Subject 1 Gait Speed Estimation Validation

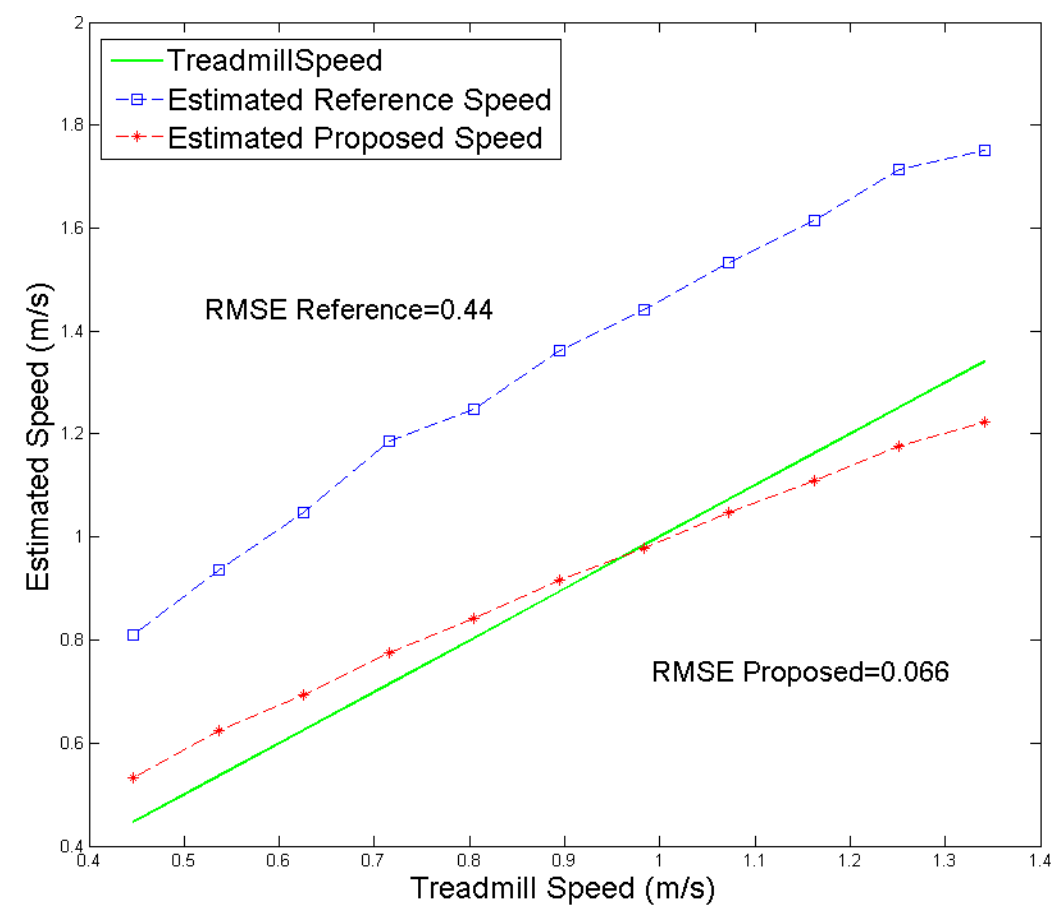

Figure 21 Subject 2 Gait Speed Estimation Validation 


\subsubsection{Discussion of Gait Speed Estimation Method using Human Gait Model}

The algorithm described above tends to overestimate at lower speeds and underestimate at higher speeds, which has also been found in [59]. Two sources of errors may lead to this: drift cancellation error and gait model error. On one hand, at low speeds, the longer gait cycle contains more integration drift, which cannot be eliminated completely by the linear drift cancelation function and leads to overestimation of the shank angle. Whereas at high speeds, the drift is overcorrected, decreasing the maximum shank angle in a gait cycle. On the other hand, the model also assumes the thigh angle is negligible during walking, i.e. it is vertical when the shank is at $\theta \max$. However, at very low and high speeds, the thigh angle can be critical for controlling the step length. At very low speeds, the thigh tends to swing forward ahead of plumb line so as to maintain a very short step length on the treadmill, resulting in a step length that is shorter than predicted, and vice versa at high speeds. Thus, correction factors are needed to further reduce errors at very slow or fast walking speeds.

These observations also shed light on the errors seen on different subjects. It is observed the algorithm tends to overestimate the gait speed of the male subject. Since the male subject is taller, at a given speed he has a lower cadence and longer stride resulting in more drift and more forward thigh when the shank is at $\theta \max$. It is, however, still possible to compensate for these inaccurate corrections with a scaling factor, since the slope of the estimated speed is fairly constant. 
Evidently, this first-principles model requires in-depth biomechanical knowledge and signal processing knowledge to extract the gait speed feature, in order to account for the noise, systematical errors etc. In Chapter 5, this gait speed extraction using firstprinciples models will be revisited and compared with a different approach - machine learning - in order to provide the insight of feature extraction.

\subsection{Summary}

This chapter details commonly used linear temporal gait features from inertial BSNs for medical research. With in-depth knowledge of human gait and signal processing, first, methods of detecting critical gait events are presented based on the observation of human gait. Second, the critical timing information was extrapolated from the timestamps of the critical gait events. Third, a first-principles modeling based gait speed estimation algorithm is developed. By leveraging biomechanical knowledge, this algorithm has improved the gait speed estimation accuracy without raising the complexity of signal processing. These linear features are important and prevailing in current gait analysis for medical research. The success of extracting such information from inertial BSNs in this chapter has demonstrated the potential of adopting inertial BSNs for such kind of gait analysis in the longitudinal studies. 


\section{Chapter 4}

\section{Nonlinear Analysis for Nonobvious Gait Feature Extraction}

Chapter 3 introduces extracting linear gait features from inertial BSN data. The motivation of extracting such features is intuitive and straightforward: since the data coming out of inertial BSNs are time series (i.e., angular velocity and acceleration over time) with strong physical implications, it is natural to adopt the common time domain analysis and detect critical turn points of the signals (i.e. gait event detection). However, such techniques have shown limitations in human biomechanical analysis, as neuromuscular control in human beings is nonlinear in nature. The resultant features usually do not reflect the characteristics of the gait patterns where biomechanical insights behind the kinematics are often desired to characterize the pathology.

To broaden the scope and extend extractable gait features from these time series, nonlinear analysis - which has established parameters to analyze a pseudo-periodical system (just like human gait) - can be borrowed. This chapter introduces nonlinear

This chapter is partially adapted from publication [P2] 
analysis tools such as Poincaré map and Lyapunov exponent for gait analysis, and extracts nonobvious gait features with insight and novelty. Since the features are by using nonlinear analysis, in this dissertation, they are categorized as nonlinear features.

\subsection{Phase Portrait}

A phase portrait is a geometric representation of trajectories of a dynamical system in the phase plane [46]. In this representation, the position information is often plotted against its first time derivative. It has been used as a visualization tool in mechanics because of the unique geometric pattern it represents for a certain dynamic system. Therefore, it can characterize the dynamic system in absence of the motion equations while the experimental data of position and its derivative are known - such as in the current inertial BSN applications.

More specifically, by directly displaying both position information and its first time derivative simultaneously, it becomes possible to correlate the two variables. For example, gait motion range is usually of interest for studies of motion constraints and amplitude [49]. Current gait analysis usually observes the amplitude of angular position and angular velocity sequentially, providing information of the extremes of amplitude per gait cycle. However, it does not reveal the mechanics behind the motion range, and rather assesses the two interdependent variables -- angular velocity and angular position -isolatedly. In gait analysis where kinematic variables must be analyzed simultaneously than sequentially, phase portrait is an intuitive way to do so. 


\subsubsection{Related Work}

Introducing phase portrait to gait analysis can be dated back to [47][48][49], where the gait patterns were studied as limit cycles from an oscillating system. Since then, the visualization of gait pattern by applying the dynamic theory attracted researchers attention. In robotics specially, limit cycles of human gait have been studied and applied to the robots for better mimicry of more natural and stable gait. [47] compared the normal gait patterns and abnormal gait patterns, "providing initial evidence about neuromuscular control features of gait" [50]. [48] qualitatively assessed the stability of the neuromuscular system by visually observing the amount of variability of the phase portrait trajectories. For example, normal gait patterns possess flexibility in the presentation of small variability in its phase portrait due to the adjustment to global and local perturbations. While for some abnormal gait patterns, excessive amount of variability was shown to be associated with instabilities and less efficient walking [50]. These pioneer work brought insight to gait analysis by applying dynamic system theory. However, for the analysis to be suitable for high precision gait analysis, methods for quantifying the phase portrait plots must be developed.

\subsubsection{Qualitative Analysis}

A shank segment phase portrait of one healthy gait cycle is plotted in Figure 14 for qualitative analysis. The gait events are clockwisely sequential on the plot as the arrows indicate. The closed curve form reveals the periodic nature of a healthy gait. The 'sharp' turning point indicates the sudden change in motion [50]. From the biomechanical point of view, a complex 'jerky' movement means inefficienty in the gait, therefore, the 
complexity of phase portrait could provide a measure of the gait efficiency. Research has also applied Ellipitc Fourier Analysis [51][52] for quantifying phase portrait complexity.

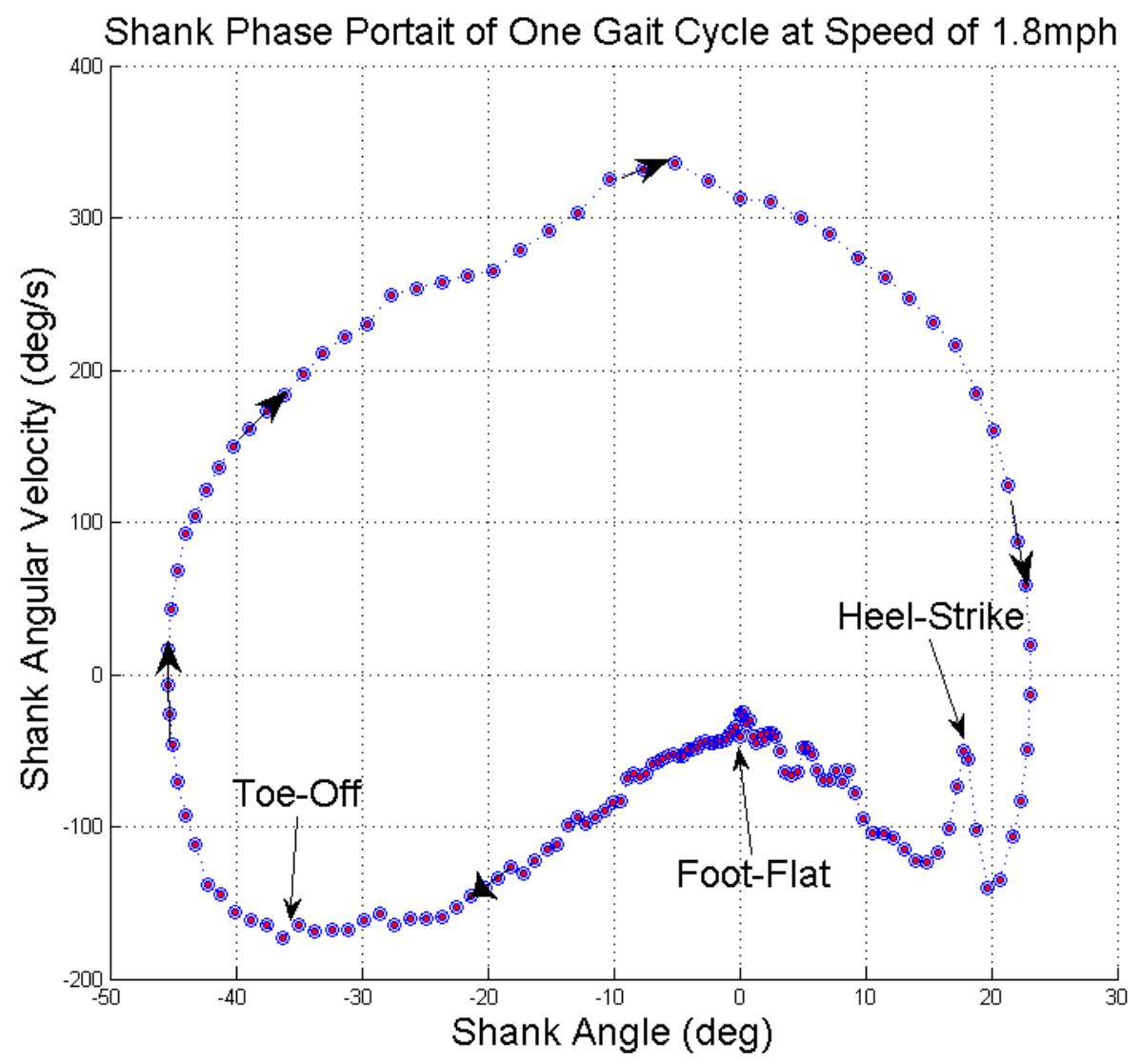

\section{Figure 22 Shank Phase Portrait of A Health Subject's One Gait Cycle}

Phase portrait does not reflect temporal informaiton. For example, the swing phase (from Toe-Off to Heel-Strike) occupies $40 \%$ of a gait cycle duration, though it may appear to be occupying most of the phase portrait circle. As shown in the scatter plot in Figure 22, the number of data points during the swing phase is less than the number of data points during the stance phase, which qualitatively indicates the duration of each gait phase, as the time interval between two data points is 1/128s (TEMPO's sampling rate was set at 
$128 \mathrm{~Hz})$. Indeed, for temporal information critical applications, this is a major drawback of phase portrait and time domain analysis is preferred. Nevertheless, the phase portrait provide a very intuitive visualization of the gait data from inerital sensors. With quantitative method to mine information from it, we can obtain features that provides much richer information than the common time domain analysis presented in Chapter 2 .

\subsubsection{Quantitative Analysis}

Although phase portrait provides visual, qualitive interpretability, to make it useful for clinical decisions, quantified information extracted from phase portrait for inertial BSN data is necessary.

\subsubsection{Complexity Analysis}

The idea of complexity analysis lies in analyzing the 'jerkiness' of a motion. This 'jerkiness' can be best shown on a phase portrait (Figure 23 and Figure 24). To quantify this complexity, we can adopt method described in [51] by computing the number of harmonics needed to fit the shape of the phase portrait. In [51], the Elliptical Fourier Analysis (EFA) was used to find the number of harmonics on 2D curves (i.e. phase portrait) needed to fit a particular phase portrait. To determine how many harmonics are required to best describe a phase portrait, [51] adopted an point-wise sum of squared errors (SSE) metric - comparing the difference between a less number of harmonics fit phase portrait to a full fit phase portrait with a number of harmonics of 500 (Note that a phase portrait with zero number of harmonics fit is a standard ellipse). Once the SSE is below a threshold, the algorithm stops searching and register the current number of 
harmonics as the quantitative complexity measure of the phase portrait. The details of this quantification method described in [51] will not be further elaborated here.

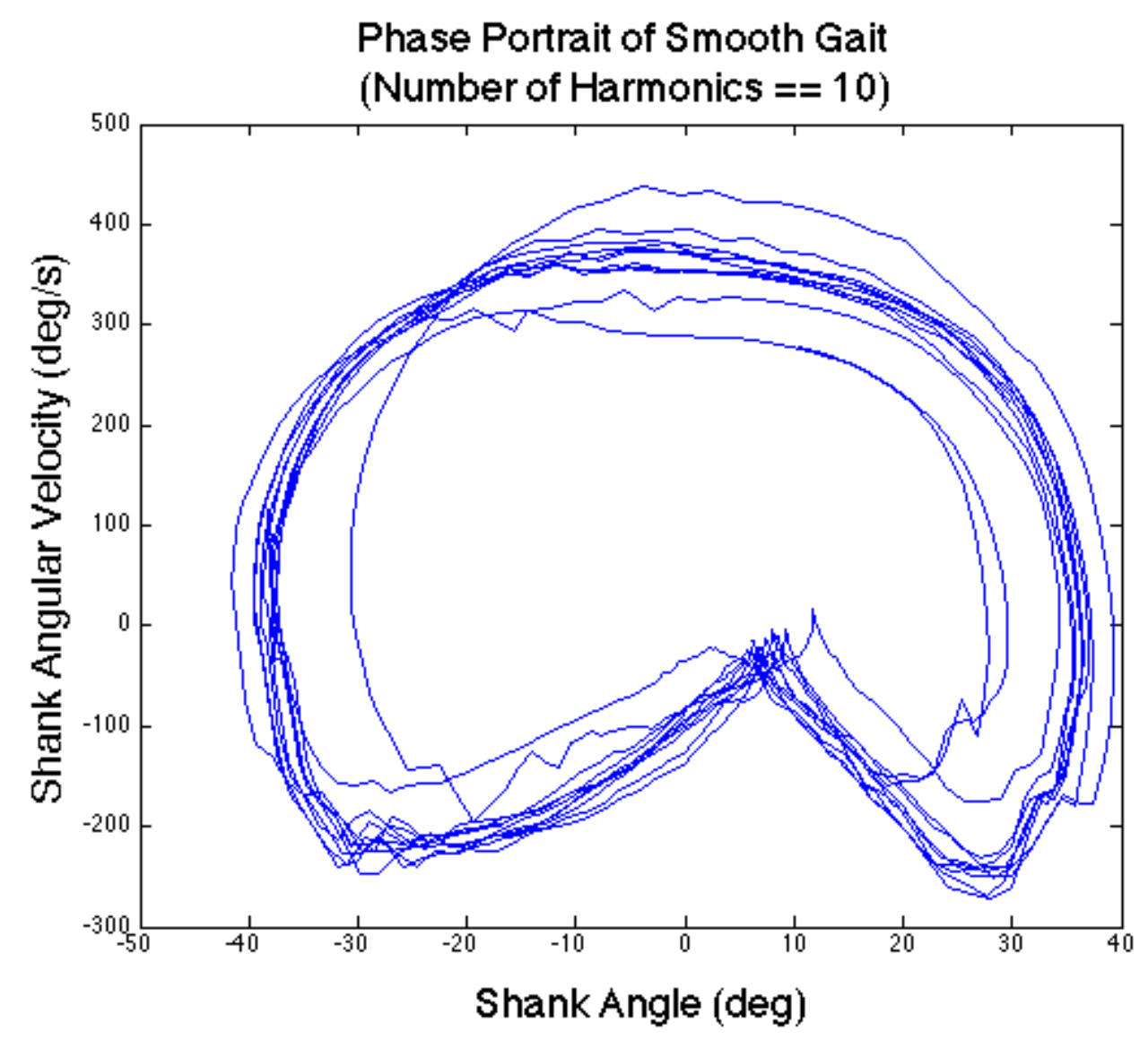

Figure 23 Phase Portrait of Less Complex Gait 


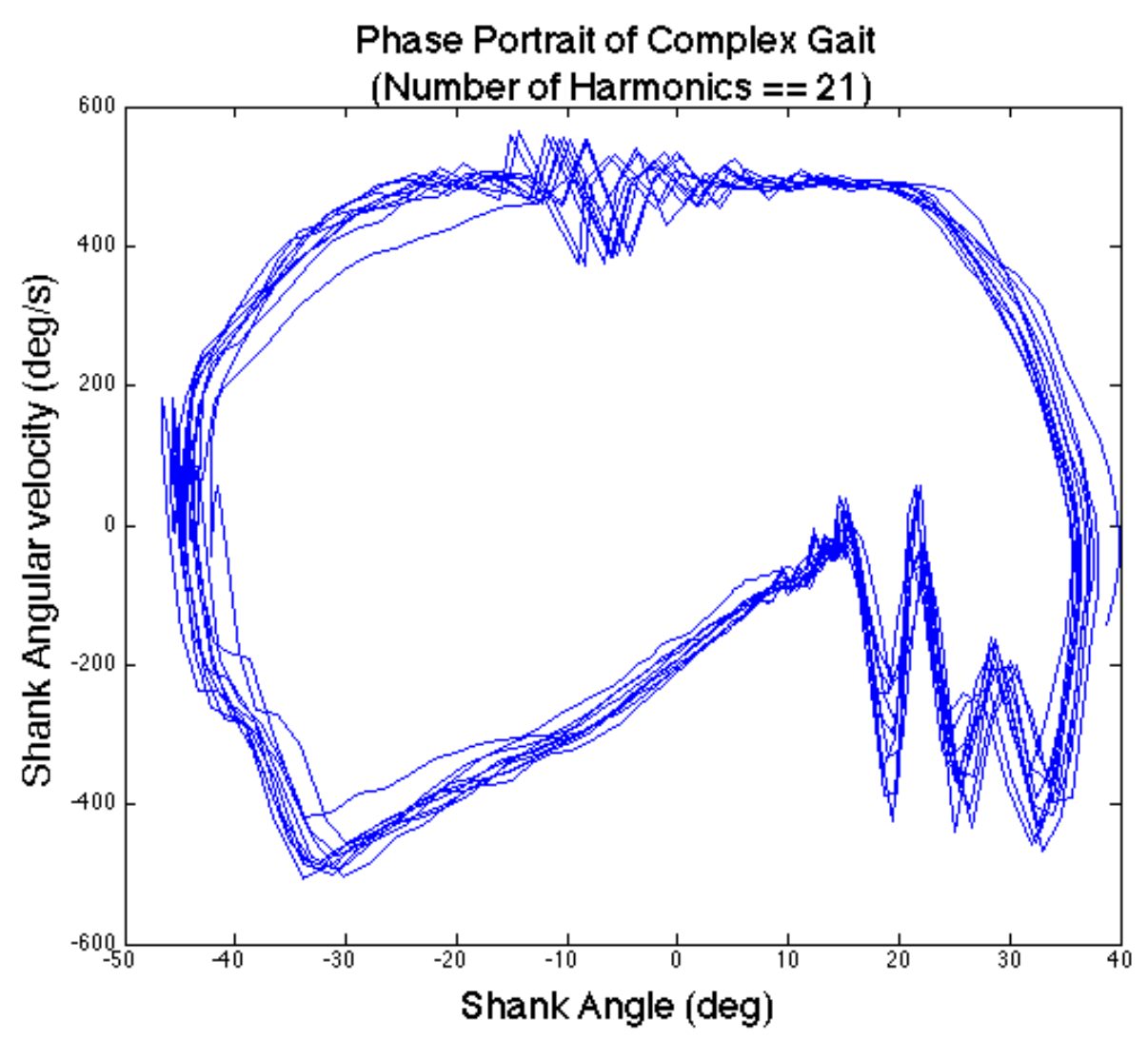

Figure 24 Phase Portrait of More Complex Gait

\subsubsection{Phase Portrait Area Analysis}

The area of phase portrait characterizes the mechanical energy spent in one gait cycle. To analytically explain this, an equation is derived relating the shank angular velocity and shank angle by modeling the shank motion during swing phase as a pendulum, as shown in Figure 25. 


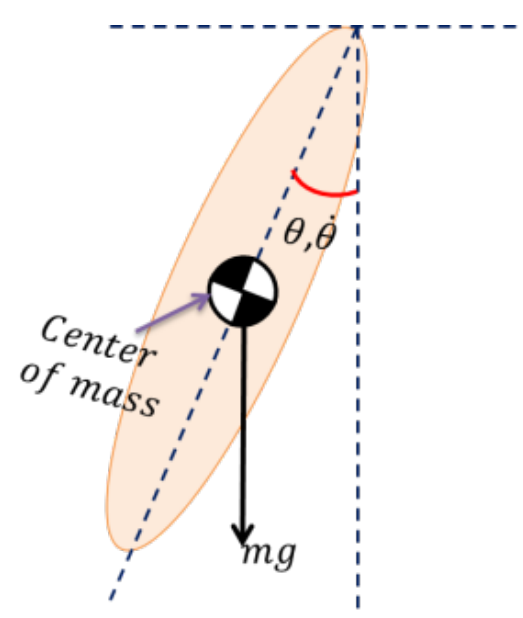

\section{Figure 25 Pendulum Model of Shank Motion}

During the swing phase, the shank can be considered as a simplified pendulum model, roughly conserving the mechanical energy generated at the push-off phase. Thus, its mechanical energy can be written as:

$$
\begin{gathered}
E=T+V \\
T=\frac{1}{2} m v^{2}+\frac{1}{2} I \dot{\theta}^{2} \\
V=m g(l-l \cos \theta)=m g l(1-\cos \theta)
\end{gathered}
$$

where

$$
\begin{gathered}
v=l \sin \theta \\
\sin \theta \approx \theta \\
\cos \theta \approx 1-\frac{\theta^{2}}{2 !}
\end{gathered}
$$

when $\theta$ is small after applying Taylor expansion. $E, T$ and $V$ represent mechanical energy, kinetic energy and potential energy, respectively. $m$ is the mass of the shank, $v$ is the 
instantaneous linear velocity of the shank (note this measure is different from gait speed), $l$ is the length of the shank, $\theta$ is the shank angle with respect to plumb line, and $\dot{\theta}$ is the shank angular velocity. By simplifying Equations (29) to (34), we can obtain:

$$
E=\frac{\dot{\theta}^{2}}{A}+\frac{\theta^{2}}{B}
$$

where A and B are coefficients given certain body parameters (i.e., m, l). Equation (35) is an ellipse function, in which the mechanical energy $E$ is related to the radius of the ellipse and, resultantly, its area. Hereto we have shown that the area of the phase portrait during swing phase represents the mechanical energy level. This corroborates the proportional relations between the mechanic energy and phase portrait area. Since walking at certain speed requires the maintenance of certain amount of mechanic energy, it is interesting to see if the phase portrait area also correlates with gait speed. 


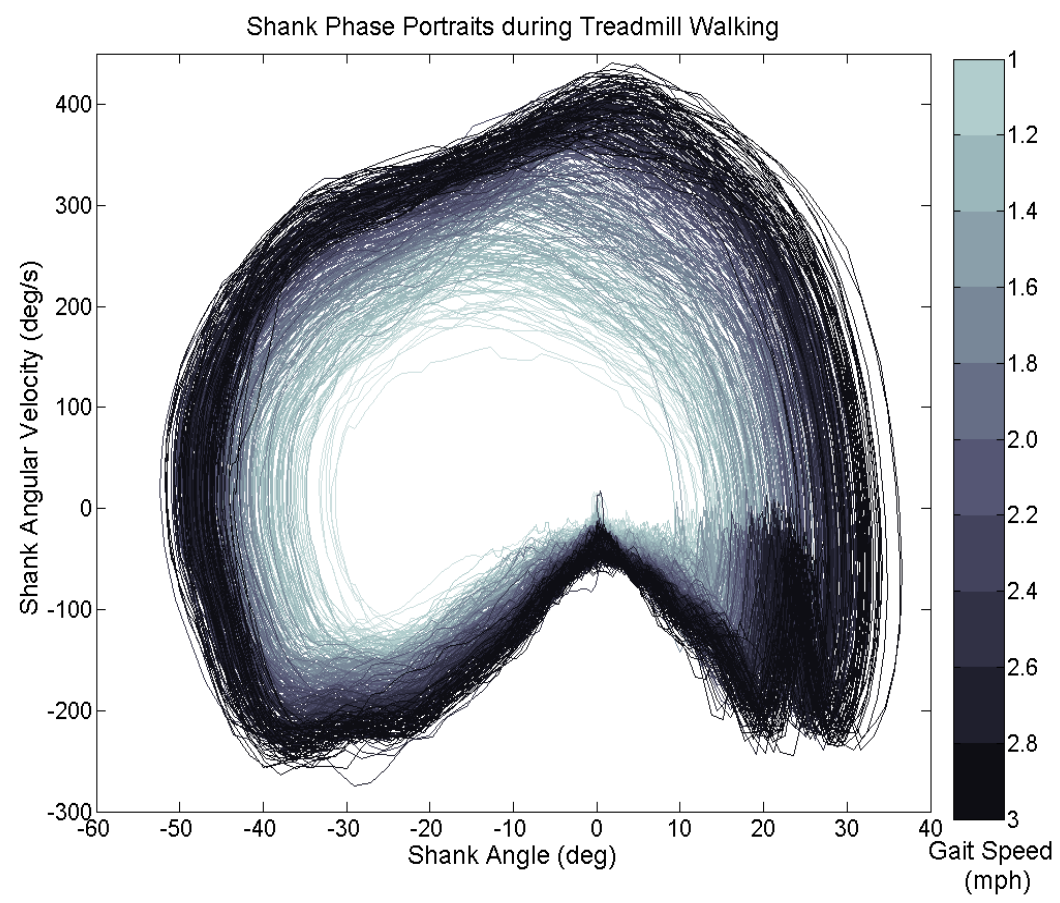

Figure 26 Phase Portraits of A Healthy Subject at Different Gait Speeds

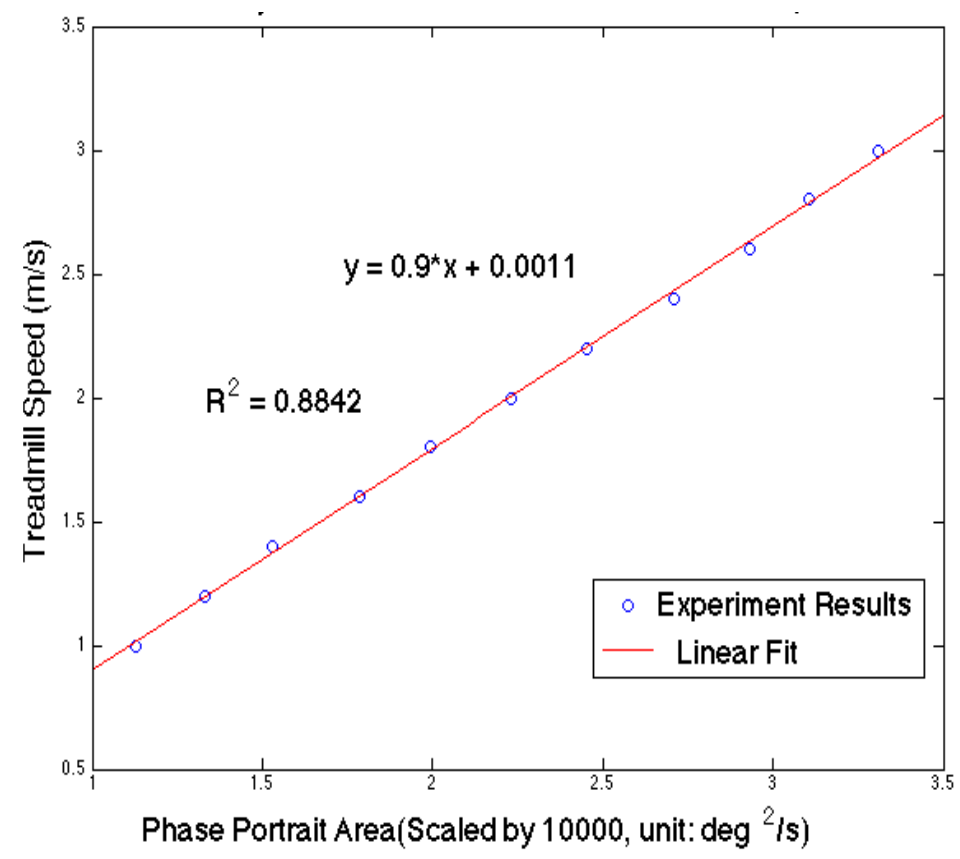

Figure 27 Regressed Linear Speed Model by Phase Portrait Area 
Figure 26 illustrates the relations between phase portrait of the shank segment and the gait speed. The darker colored phase portrait with a larger area indicates a faster walking speed on the treadmill and, consequently, with higher mechanic energy to maintain during each cycle. The metric of phase portrait area is profoundly rooted in physical models as described above. Meanwhile, it possesses very good linear relationship with gait speed, as shown in Figure 27 (average phase portrait area per treadmill session vs. treadmill speed). This property will be explored in Chapter 5 and used for gait speed extraction.

\subsection{Poincaré Map for Orbital Stability Assessment}

Poincaré map (also known as first return map) has been used to analyze orbital stability) [53], and can be applied to assess variability between gait cycles as well. A Poincaré map samples a particular event in every cycle in a cyclic signal. This concept can be illustrated as shown in Figure 28.

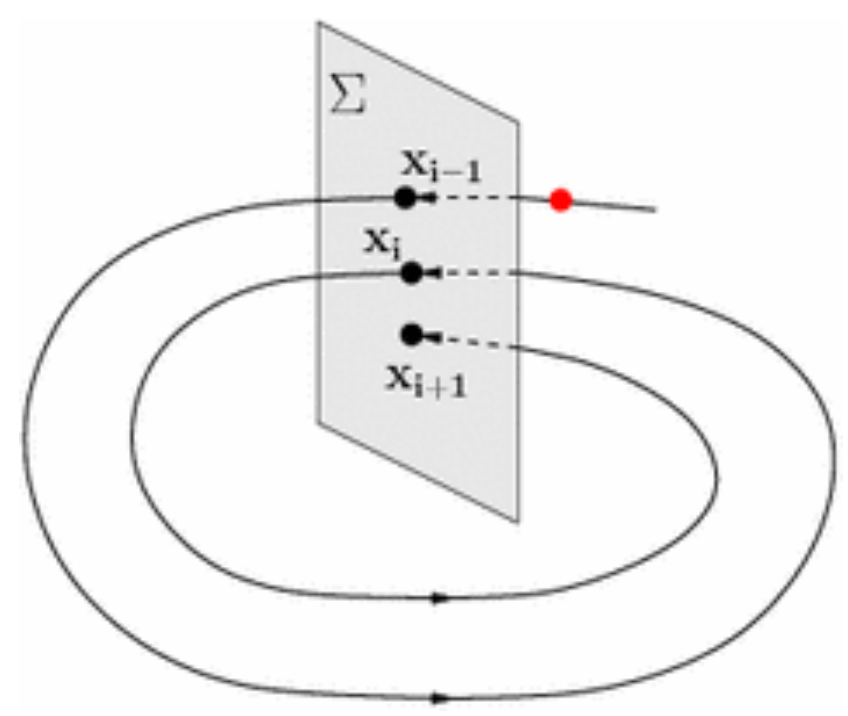




\section{Figure 28 Concept of Poincaré Map}

In gait analysis in particular, gait events occur repetitively in gait cycles. Since the data obtained from inertial BSNs is discrete time series, in order to obtain the map, the magnitude of a gait signal at that particular event of interest can be sampled as a means of assessing the orbital stability of this signal. Therefore, the task become identifying the critical gait events in time domain as described in Chapter 3, Section 3.1. As shown in Figure 29, take shank angle for example as a gait signal, the mapping plots the shank angle at toe-off moment in the previous gait cycle against the shank angle at the current toe-off event in the next gait cycle. The more clustered these return points are (red dots in Figure 29), the more orbital stability the signal possesses.

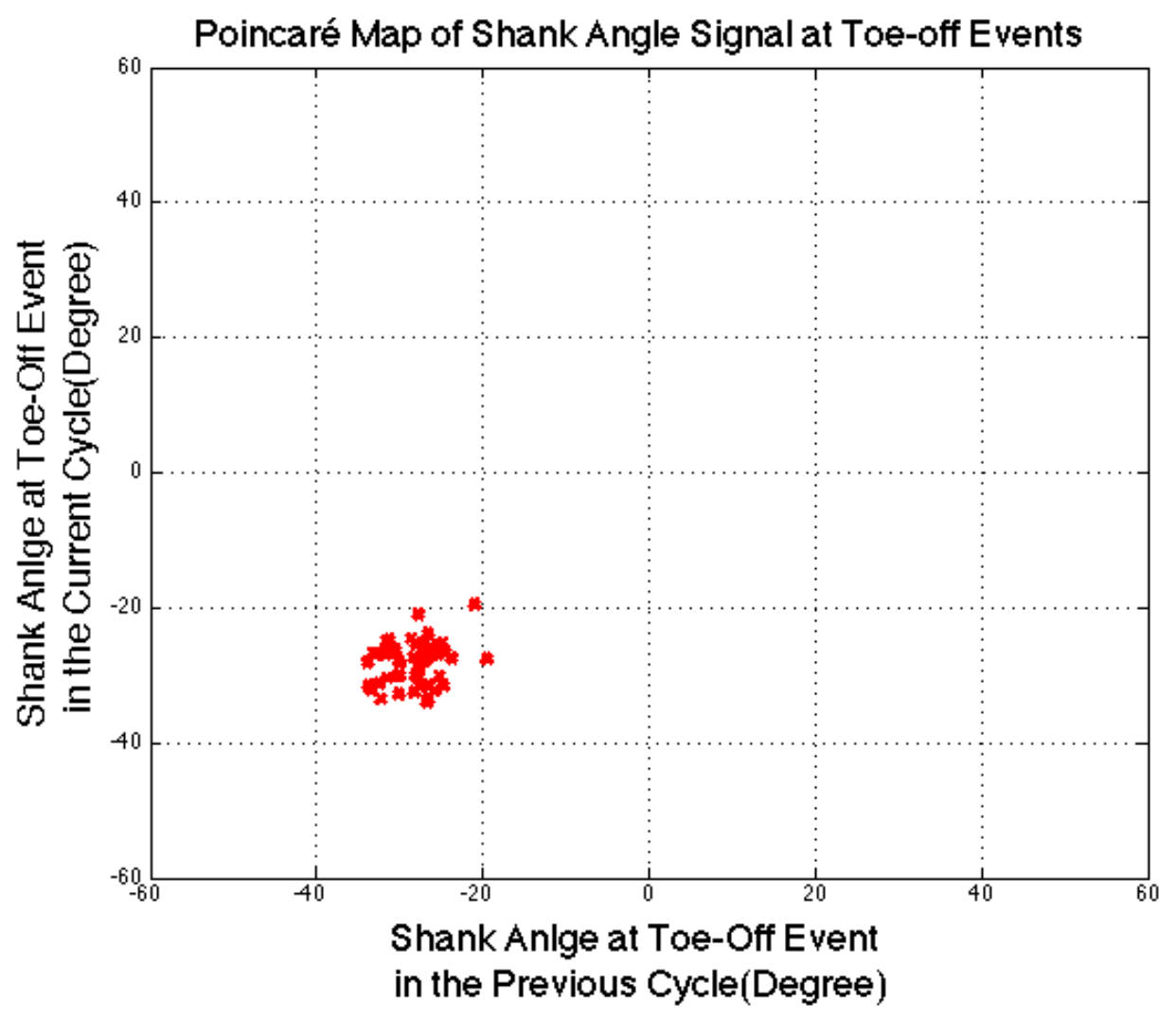


Figure 29 Example of Poincaré Map of A Gait Signal

\subsection{Lyapunov Exponent for Stability Assessment}

Gait stability is something difficult to quantify yet important to clarify. For pseudoperiodic systems as human movement function, local stability can used to describe how the dynamic system responds to "very small perturbations continuously in real time" [54]. Lyapunov exponent (LyE) is such a parameter to quantify the local stability.

To compute LyE for given time series data, the first two steps consist of finding the embedding dimension and time delay lag for phase space reconstruction. To find the embedding dimension of the time series, a False Nearest Neighbor (FNN) algorithm [55] is applied. With this algorithm, the time series of the shank's angular position is identified to have an embedding dimension of 2 . The time delay is determined by using average mutual information, and a time delay of 10 samples is usually chosen for extracting LyE from shank angular velocity signal.

After the phase space is reconstructed, LyE can be computed using either the Walgorithm [56] or the R-algorithm [55]. In gait analysis, the R-algorithm is more commonly used due to claimed accuracy on short-term data. However, [57] has argued that the $\mathrm{W}$-algorithm is more appropriate for assessing local dynamic stability because of its sensitivity in estimating LyE. Since the purpose of this paper is separating the before and after HVLP gait parameters, higher sensitivity means better separability, thus the Walgorithm will be used. The equation for computing the largest LyE using the Walgorithm is shown in Equation (36), 


$$
\lambda_{1}=\frac{1}{N \times \Delta t} \times \sum_{i=1}^{M} \log _{2} \frac{L^{\prime}(i)}{L(i)}
$$

wherein $\lambda_{1}$ is the largest LyE in bits/seconds. $\Delta t$ is the sampling period, in our case, $1 / 128$ second. $N$ is the total number of steps on the fiduciary trajectory. $M$ is total number of replacement steps. $L^{\prime}(i)$ and $L(i)$ are the distances between the vectors at the beginning and end of a replacement step, respectively. In general, the W-algorithm tracks and quantifies the divergence of the nearest neighbors in the trajectories in the reconstructed phase space. First, it selects a fiduciary trajectory with a single nearest neighbor being followed and replaced when its separation $L^{\prime}(i)$ from the fiduciary trajectory becomes large [57]. A new neighbor on the fiduciary trajectory is then selected to minimize the length $L(i)$ and the angular separation estimated by directional cosine value. This procedure is repeated until the fiduciary trajectory has gone over the entire data set. Then the largest LyE is obtained as shown in Equation (36).

\subsection{Summary}

This chapter explored the possibility of applying nonlinear analysis tools for the kinematic data obtained from inertial BSNs. Nonlinear features are features capturing characteristics of a dynamic system. Unlike linear features, these features do not possess superposition properties, i.e. linearity. As human gait is such a dynamic system that is not determined well by linear rules, it is intuitive to characterize such system with chaos theory. To gain insight behind the kinematic information, shank phase portraits were studied and quantified with gait features that are potentially useful for gait analysis using 
inertial BSN data. Compared to conventional feature extraction for activity classification using inertial BSNs, this approach provides biomechanical insight from a nonlinear dynamics perspective. With broadening the scope of feature extraction from inertial BSNs, a new set of features can be extracted using nonlinear analysis and may further enhance classification accuracy. 


\section{Chapter 5}

\section{Introducing Machine Learning Techniques for Feature Extraction}

Chapter $2 \sim$ Chapter 4 summarizes the techniques for gait feature extraction within a system from inertial BSN data. These techniques are all based on first-principles models, which derive models from fundamental physics without statistical learning. Although these first-principles models provide the insight, it is also useful to employ machine learning techniques to boost up the accuracy, simplify the experiment design and generalize the post-processing approach. In this chapter, we explore the possibility of adopting machine learning for one gait feature extraction - gait speed - to exemplify that information extraction can benefit from combining machine learning techniques and firstprinciples modeling. By studying the features that can be used for gait speed estimation, machine learning provides a general framework to select feature, and correct systematical errors that inevitably remain by solely relying on first-principles modeling.

\footnotetext{
This chapter is partially adapted from publication [P2]
} 


\subsection{Advantages of Combining Machine Learning with}

\section{First-Principles Modeling}

In Chapter 3, Section 3.2, a first-principles based model for gait speed extraction is described. We have also found that systematical error still exists in the final results with a series of correction mechanism such as calibration, mounting calibration and an improved double pendulum model. Apparently, other gait speed extraction methods based on firstprinciples modeling can still be explored [37][58][59], but none of these methods can achieve accurate results without in-depth analysis and modeling, and different correction procedures at certain steps of the whole process for minimizing the final errors for the following reasons:

1) Sensing modality of inertial BSN - since its sensing nature is derivative of spatial information and the intrinsic senor noise [37], transforming the derivatives to get spatial information is challenging and subject to errors.

2) Generalization of model, because of the individual difference and complexity of human gait, individual parameters are usually required (e.g. leg length as in [37] and [59]) for first-principles models. Such parameters, not only cause burden for the users in the real-world deployment, but also can bring in impactful measurement errors for the estimation system.

In a framework based on first-principles models, different calibration procedures are usually applied to minimize these measurement errors along with the systematical errors at each step of the workflow. Therefore, it is more appealing to adopt a framework that 
accounts all such errors through a generalized calibration procedure than adopting the first-principles models system for future real-world deployment. Machine learning approaches, on the other hand, can account for parameter difference by learning the training data without relying on complex gait model, and hence are implemented more in studies of gait speed estimation using inertial sensors [60].

\subsection{Feature Identification}

This section explores and identifies features that can be extracted from inertial sensors for gait speed estimation. Features that are intuitively related to gait speed are firstly presented. Then we use a novel nonlinear feature that is rooted in analytical mechanics. All the features extracted and operated on per gait cycle basis. In other words, each data point contains features extracted from one gait cycle's data.

\subsubsection{Linear Features}

Some of the linear features can be identified based on intuition of variables related to gait speed. For example, cadence is selected for gait speed estimation for either its accessibility (i.e. accelerometer-only sensor platform) or its apparent link to gait speed (i.e. it is self-evident that faster pace results in higher gait speed). This information will be included in our linear feature set.

Linear features can also be extracted by generalizing the variables from first-principles models. Recall Chapter 2, Section 3.2, we learned the variables involved in the gait speed algorithm include the leg length $\left(L_{L e g}\right)$, shank length $\left(L_{\text {Shank }}\right)$, and maximum shank angle 
during the forward swing $\left(\theta_{\min }\right)$ and backward swing phase $\left(\theta_{\max }\right)$, as shown in Figure 19. For a particular individual, the variable will be $\theta_{\min }$ and $\theta_{\max }$ since body parameter does not vary. We also include two of the common statistical measures in our study: range of angular velocity and range of angular position per gait cycle for testing the algorithm of gait speed estimation. Thus in total, there are five linear features evaluated in machine learning algorithm: sine of $\theta_{\max }$, sine of $\theta_{\min }$, time span of a gait cycle, range of angular velocity and range of angular position per gait cycle.

\subsubsection{A Nonlinear Feature}

In Chapter 4, Section 4.1.3.2, we identified a quantified phase portrait measure - area of phase portrait, which possesses good linear relationship with gait speed (see Figure 26 and Figure 27). Despite the discovered high correlation shown between this feature and gait speed regardless of the individual parameters (e.g. shank length and leg length), without obtaining a training set of data (i.e. with gait speed ground truth as reference) and applying regression or statistic learning method, this feature can not predict gait speed alone. Therefore, machine learning method can be very handy for adopting such features - which cannot predict based on first-principles model alone - into feature extraction framework.

\subsection{Feature Selection}

Feature selection is an important step in machine learning since the accuracy of the model generated by machine learning usually depends on the dimension of the feature space, where the rule of thumb is ratio of the number of instances $\mathrm{N}$ in the training data set over 
the number of features $D-N / D-$ must be at least ten. In this section, both linear features and the nonlinear feature will be used and selected by using machine learning algorithm.

\subsubsection{Feature Importance}

We compare both linear and nonlinear features extracted for gait speed estimation in this section. First, the correlation between features and gait speed are examined. Feature selection algorithm is also used to vote the most important feature. Second, to evaluate the performance of the features in regression, intra-subject cross-validation is used to compare accuracy and training size. The results are shown as follows.

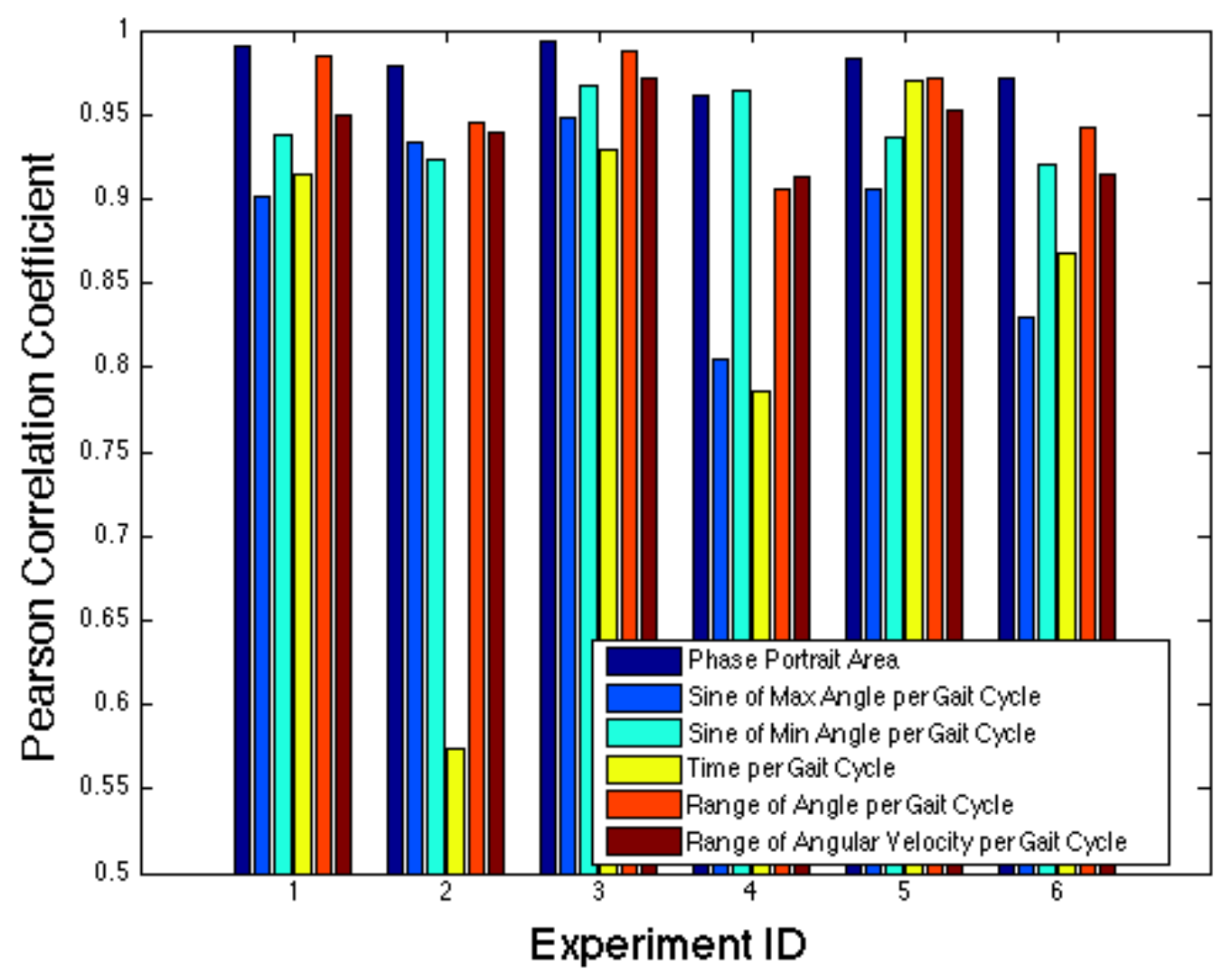

Figure 30 Correlation Coefficients between Different Features and Gait Speed. 
Figure 30 shows that the phase portrait area has become the most correlated feature with gait speed in comparison to other linear features. Across all the experiments, phase portrait area provides an average correlation coefficient of 0.9799 , which is 0.04 point higher than the linear feature with second highest correlation among all features.

\subsubsection{Automatic Feature Selection}

To further explore the automation possibility of adopting machine learning framework, automatic feature selection algorithm such as ReliefF [61] and LASSO [62] are applied to select the most predictable features. Both algorithms have ranked the nonlinear feature as the most important feature for predicting gait speed, which conforms to the highest correlation finding, and demonstrates the importance of extracting the predictable features via first-principles modeling.

\subsection{Evaluation}

Knowing that the nonlinear feature provides the highest correlation with gait speed, we feed the features into several machine learning algorithm to test their performance in regression. Learning algorithms including Naïve Bayesian (Bayes) [63], Random Forest (Bagged Tree) [64], Boosted Tree [65], Multivariate Adaptive Spline Fitting (MARS) [66], Multi-linear Regression (MLR) [67], and K-Nearest Neighbors (KNN) [68] are used to compare feature performance. All the learning algorithms are using default parameters from the Statistics Toolbox in Matlab ${ }^{\circledR}$. 


\subsubsection{Comparison Based on Machine Learning}

Intra-subject validation is applied to simulate the scenario where an individual user is required to conduct a pre-use data collection after system deployment. In the process of the 'holdout' validation [69], a certain amount of data is left out for the training set (preuse data collection stage after deployment), and the rest is used as testing set (system in use after deployment). To find out how much training data is required in order to produce accurate results, training set percentage is set ranging from $10 \% \sim 90 \%$ of the entire data at $10 \%$ interval (equivalently around 0.5 minute). The results are shown from Figure 31 to Figure 33.

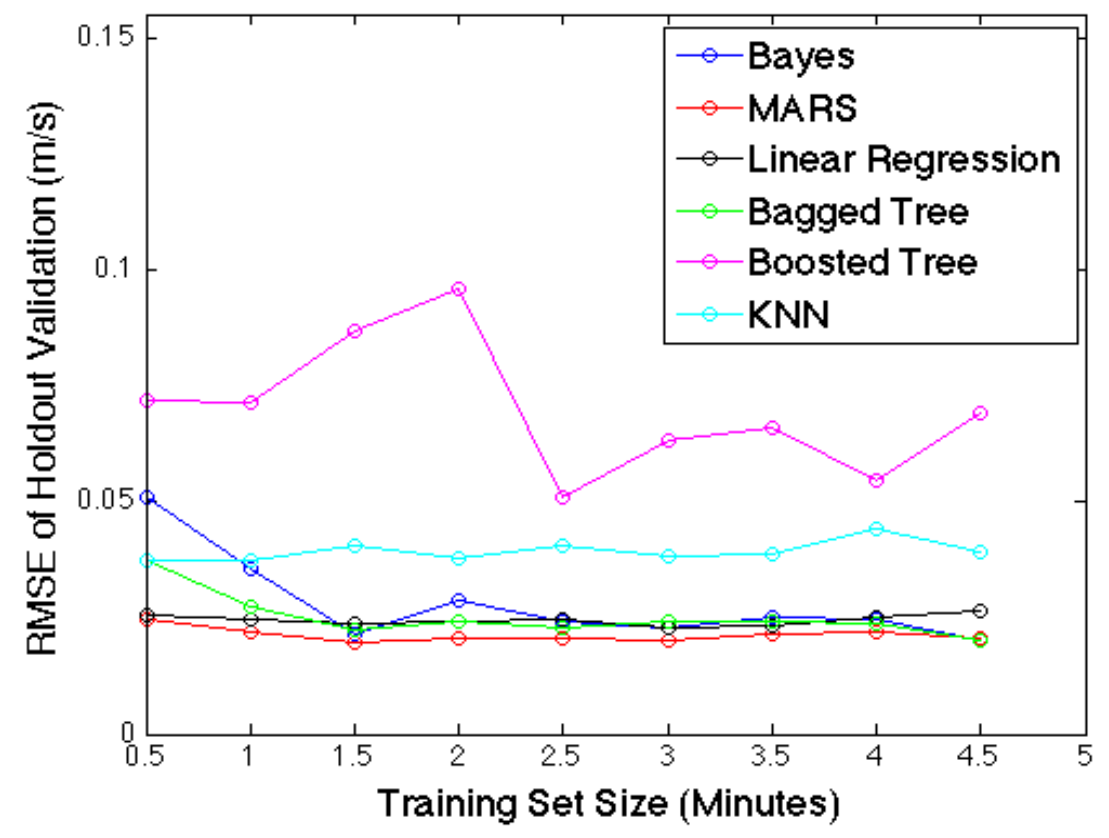

Figure 31 Intra-subject Validation with Linear Features

Comparing Figure 31 and Figure 32, both linear features and the nonlinear feature have provided good accuracy for intra-subject learning. We also find that using the nonlinear 
feature can achieve similar accuracy as using the combination of linear features. Plus, with less training data $(\sim 10 \%)$, the nonlinear feature alone can estimate the gait speed as accurately as the linear feature combinations.

Figure 33 further evaluates the feature performance with linear regression (commonly used for its simplicity) as the learning method. The results show that the nonlinear feature alone achieves an RMSE of $0.189 \mathrm{~m} / \mathrm{s}$, surpassing using all linear features together (with an RMSE of $0.024 \mathrm{~m} / \mathrm{s}$ ). This feature alone also provides more interpretable results (rooted in fundamental mechanics) in comparison to combined linear features. Moreover, adding the nonlinear feature to the linear feature set will increase the accuracy by $25 \%$ than using linear features together.

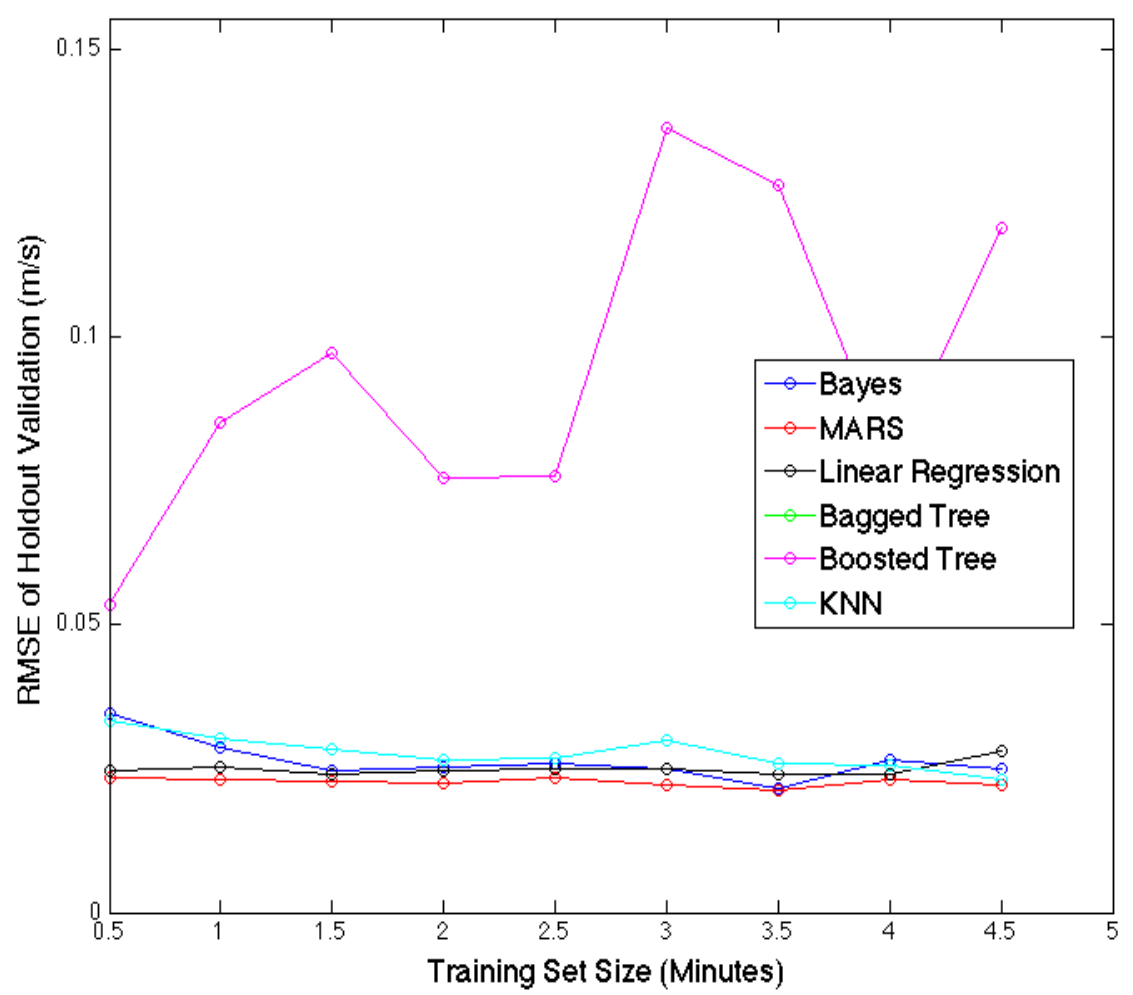

Figure 32 Intra-subject Validation with One Nonlinear Feature 


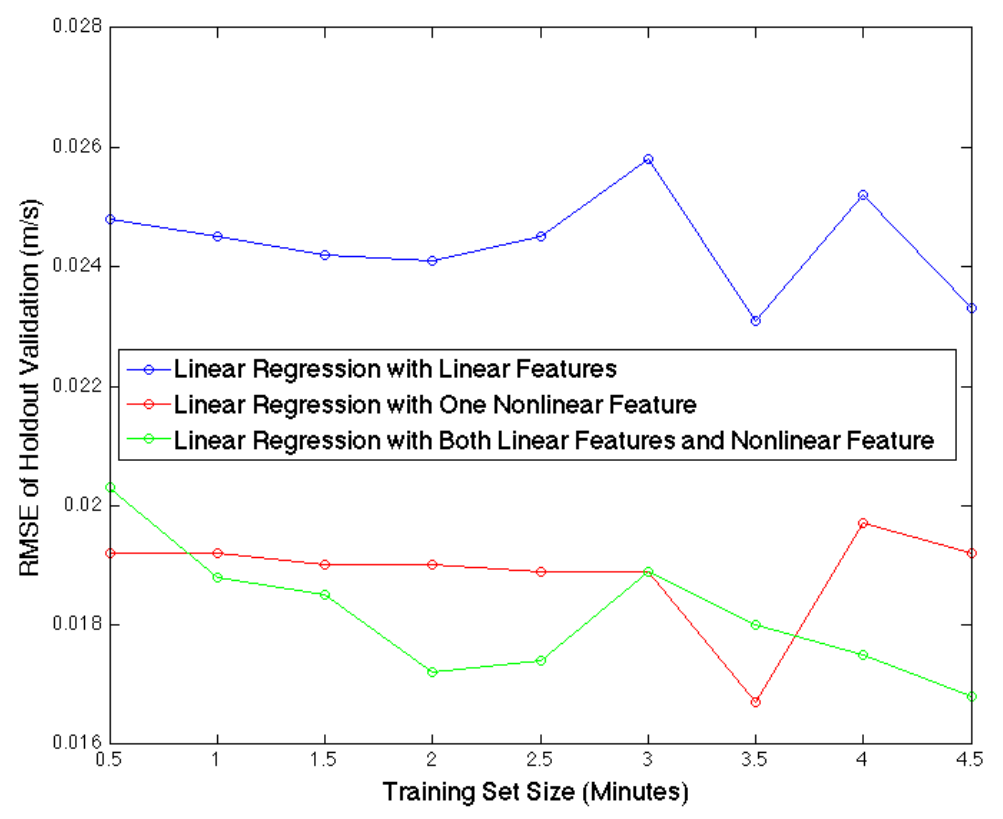

Figure 33 Inter-subject Validation with Linear Regression

\subsubsection{Diagnostics of the Linear Regression Model}

Multiple linear regression model is generate under 4 assumptions: 1) The residual values are independent 2) The predicted values can be expressed as a linear function of the variables 3) variation of observations around the regression line (the residuals) is constant, which is also know as the homoscedasticity assumption 4) The residual values are normally distributed. To check these assumptions, the diagnostics of the residual errors can be plotted for examination. To check assumption 1) and assumption 2), the residual errors can be plotted against the predicted values to observe whether pattern exists on this plot. To check assumption 3), the equal variance can be observed also on the plot of the residual errors against the predicted values. To check assumption 4), the histogram of the residual errors and the quantile-to-quantile (Q-Q) plot of the residuals against a theoretical normal distribution can be plotted to observe whether the quantile of the 
residual errors follow a linear diagonal line on the plot. The results of the examination on the linear regression model generated from $30 \%$ of the training data and evaluated on the left $70 \%$ data are shown from Figure $34 \sim$ Figure 36.
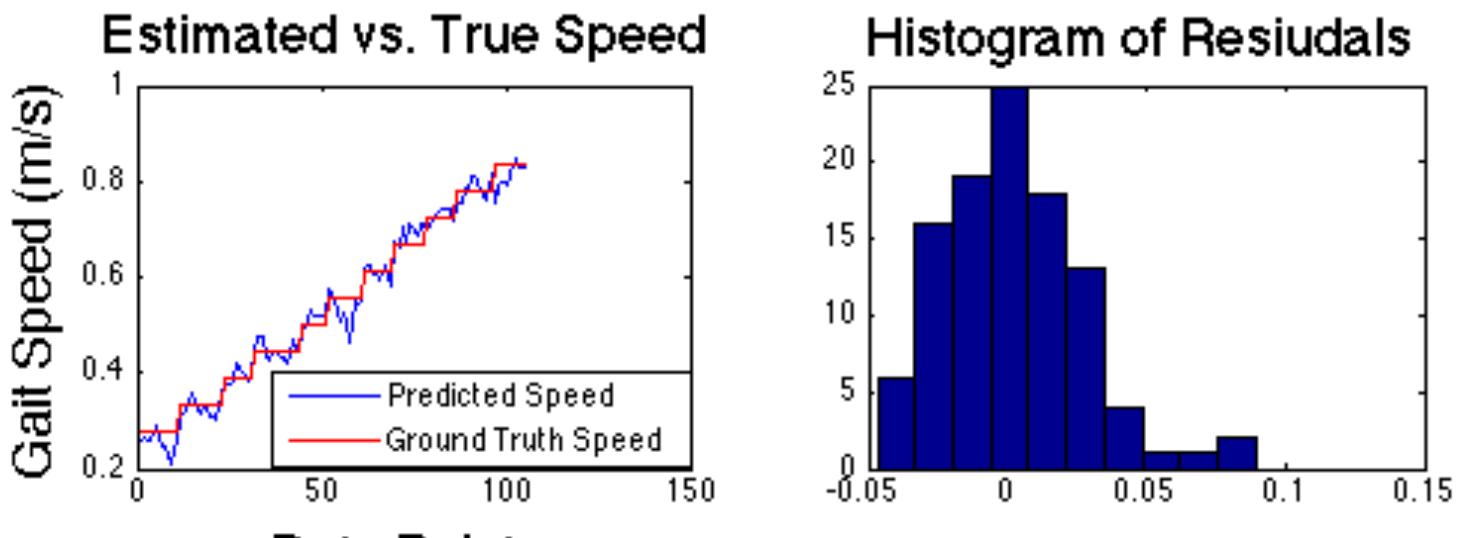

Data Points
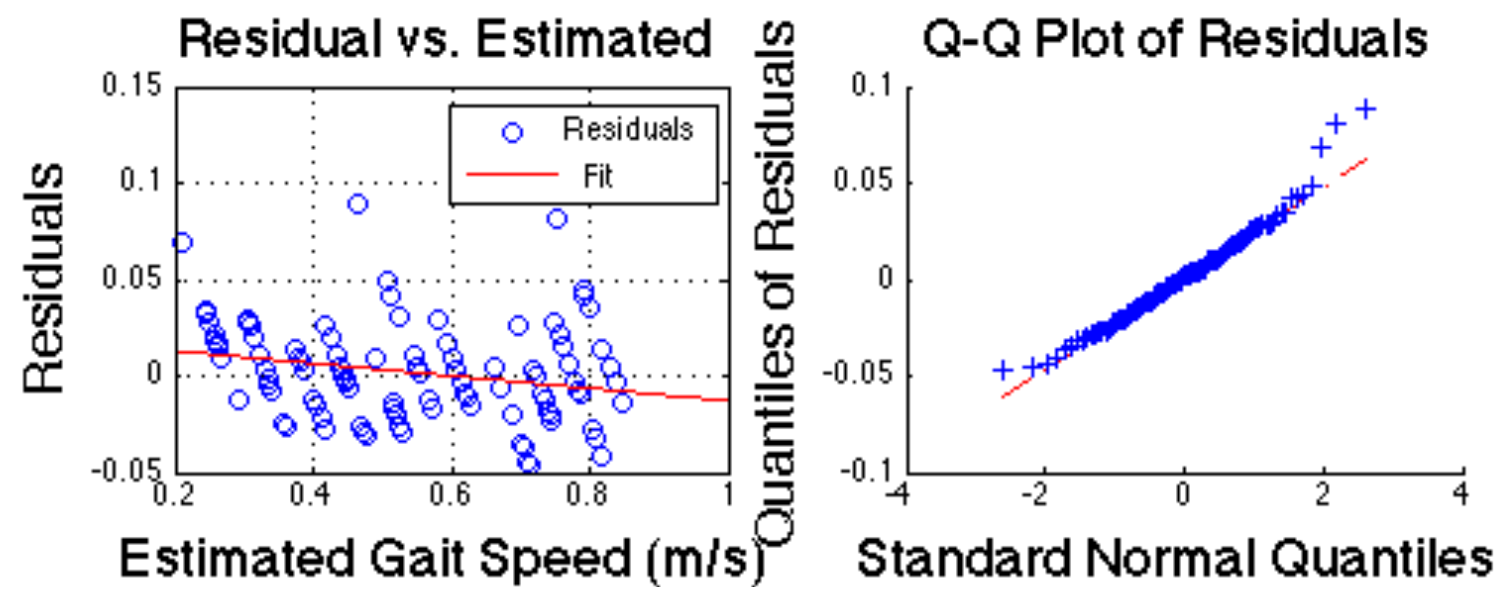

Figure 34 Diagnostic Plot of Linear Regression with Linear Features

Figure 34 shows the diagnostics of the linear regression model using the linear features.

The histogram plot and the Q-Q plot of the residuals show that the distribution of the residuals are almost normal, but contains outliers at the tail of the distribution. Because that ground truth speed at one treadmill session is a constant number while the estimated 
speed is continuous, the points on the Residual vs. Estimated plot are slightly tilted, and this tilt disappears if a white noise is added to the ground truth speed. Nevertheless, the relative flat of on the Residual vs. Estimated plot shows the variance of the residual errors are equal.
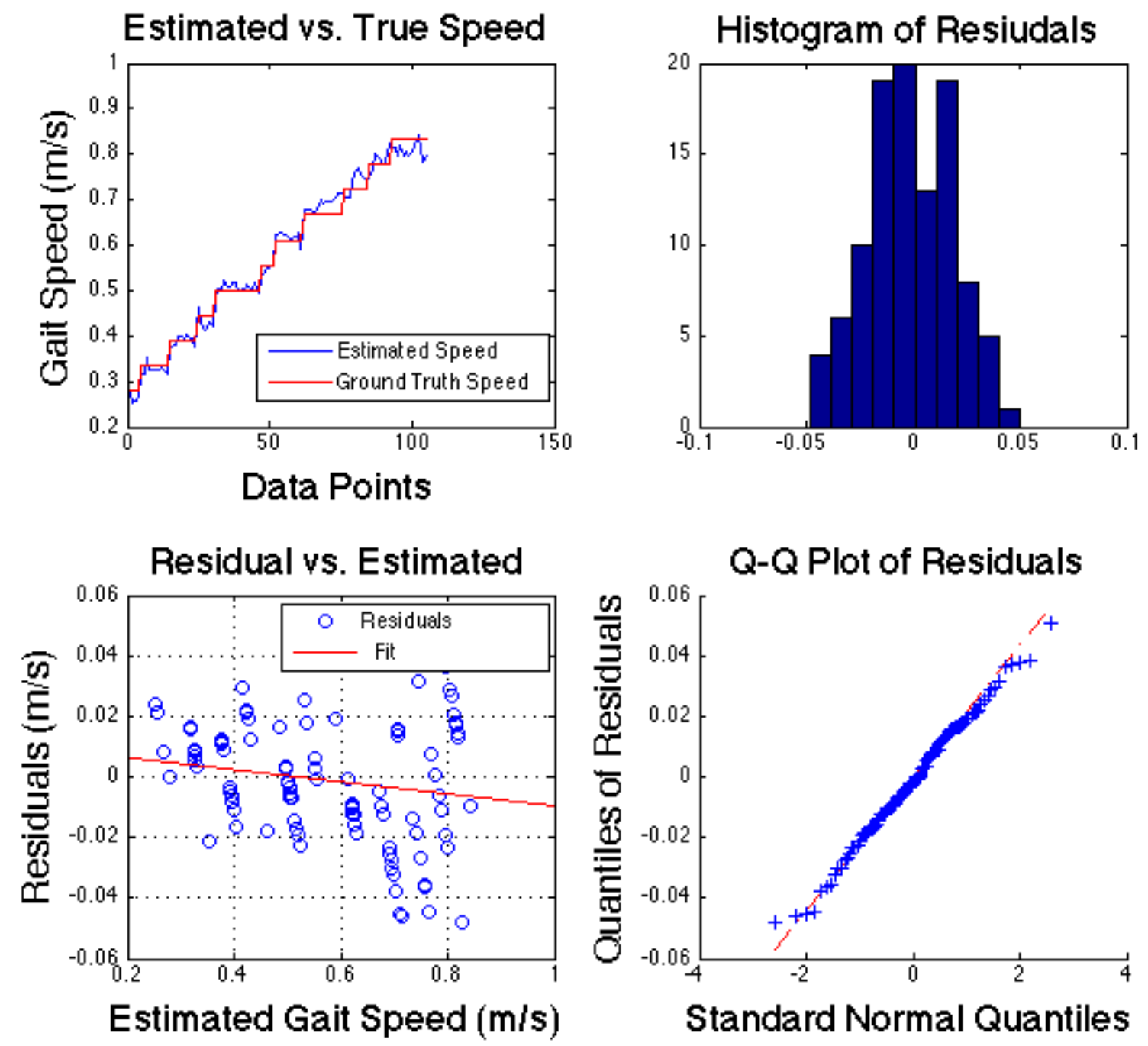

Figure 35 Diagnostic Plot of Linear Regression with One Nonlinear Feature

Figure 35 shows the diagnostics of the linear regression model using the linear features. The histogram plot and the Q-Q plot of the residuals show that the distribution of the 
residuals follows normal distribution with few outliers than the linear regression model using linear features. The Residuals vs. Estimated plot also shows that the variance of the linear model satisfies the assumption 3).
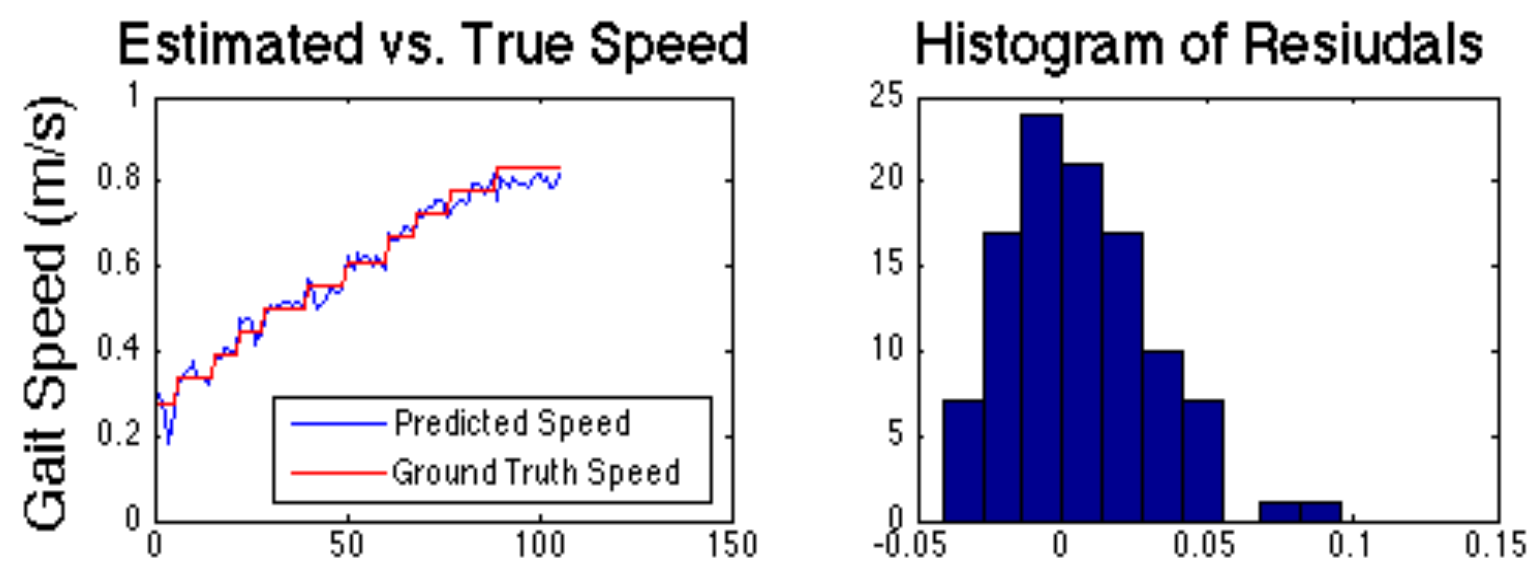

\section{Data Points}

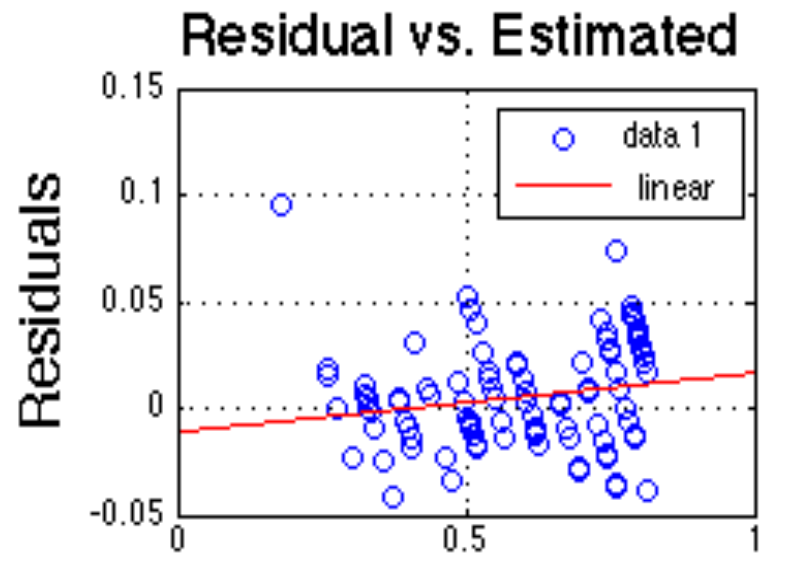

Estimated Gait Speed ( $\mathrm{m} / \mathrm{s})$

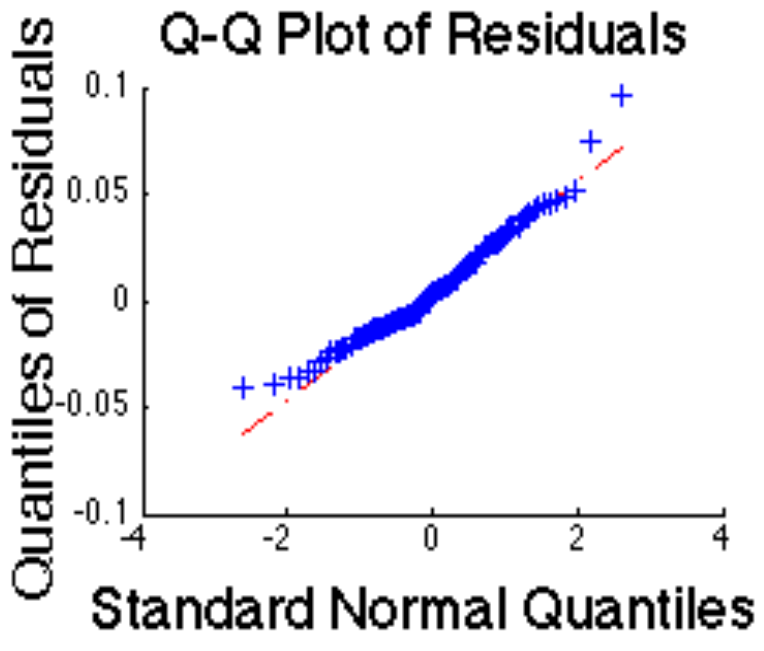

Figure 36 Diagnostic Plot of Linear Regression with All Features

Figure 36 shows the diagnostics of the linear regression model using the linear features. Again, the histogram plot and the Q-Q plot of the residuals show that the distribution of 
the residuals is almost normal, but contains outliers at the tail of the distribution as the model of using all features.

From the Q-Q plots of all three models, the linear regression model using nonlinear feature has shown the least number of outliers on the diagonal line, indicating the residual errors from this model can be better approximated as normal distribution. Also, the range of the residual errors shown on the Residual vs. Estimated plots, indicates the nonlinear feature generated model produces few residual errors and outliers in prediction.

\subsubsection{Discussion}

The insight provided in Chapter 4, Section 4.2 shows that the phase portrait area intuitively correlates with both angular position information and angular velocity information in a nonlinear mapping. In comparison, existing linear feature extraction (e.g., range of angular velocity, range of angular position, sinusoidal function of maximum angular position during each gait cycle) observes the amplitude of angular position and angular velocity sequentially, providing only information of the extremes of amplitude per gait cycle instead of the whole picture of a gait cycle. Such method does not reveal the mechanics behind the motion range, and rather assesses the two interdependent variables -- angular velocity and angular position -- isolatedly. In contrast, the phase portrait area takes into account of the sample-by-sample signal amplitude level that is bounded by mechanic fundamentals, and is proven to be better correlated with gait speed. This correlation advantage of the nonlinear feature, ultimately, boosts the estimation accuracy for the regression algorithm. 


\subsection{Summary}

This chapter explores the possibility of combining first-principles modeling and machine learning for gait feature extraction from inertial BSN data. With an exemplar gait feature - gait speed - a spatial information related parameter whose accuracy can subject to systematical error after first-principles modeling, this chapter shows the advantage of the combining - insight provided by first-principles modeling to discover high correlated factor for prediction, and the general framework provided by machine learning - by exploiting the correlation, selecting the most correlated features and correcting systematical errors remaining after first-principles modeling. This hybrid approach inspires future exploration in feature extraction from inertial BSN data. 


\section{Chapter 6}

\section{Case Studies in Medical Research}

Previous chapters have detailed the theories and techniques for gait feature extraction from inertial BSN data, laying the groundwork for searching for such techniques within a system and applying them in the real world medical applications. To test these techniques against the real world challenges, this key chapter demonstrates the feasibility of BSN enabled technology through a series of case studies: assessing efficacy of ankle-foot orthoses for children with cerebral palsy, aiding diagnosis of normal pressure hydrocephalus, and enhancing gait assessment for early multiple sclerosis. These three examples covers the areas in medicine that could benefit where high precision gait analysis is much needed in the longitudinal scale and natural environment. With real patient data collected from medical pilot studies and clinic setting, gait features are identified and extracted, demonstrating inertial BSNs' potential for propelling gait analysis involved medical research.

This chapter is partially adapted from publication [P8][P7] and [P1] 


\subsection{Orthoses for Children with Cerebral Palsy}

\subsubsection{Medical Background}

Cerebral Palsy (CP) (Figure 37) is a neuromuscular disorder characterized by an injury to the immature central nervous system (CNS). There is a wide spectrum of clinical manifestations in $\mathrm{CP}$, including spasticity, abnormal reflex patterns, weakness, retention of primitive reflexes, and an abnormal increase in muscle tone. Based on the severity and location of the CNS lesion, children range from being wheelchair-bound to community ambulators. In addition, many patients develop muscle contractures during growth, resulting in joint stiffness as well as angular and rotational bony deformities. As a result, these children develop atypical gait patterns.

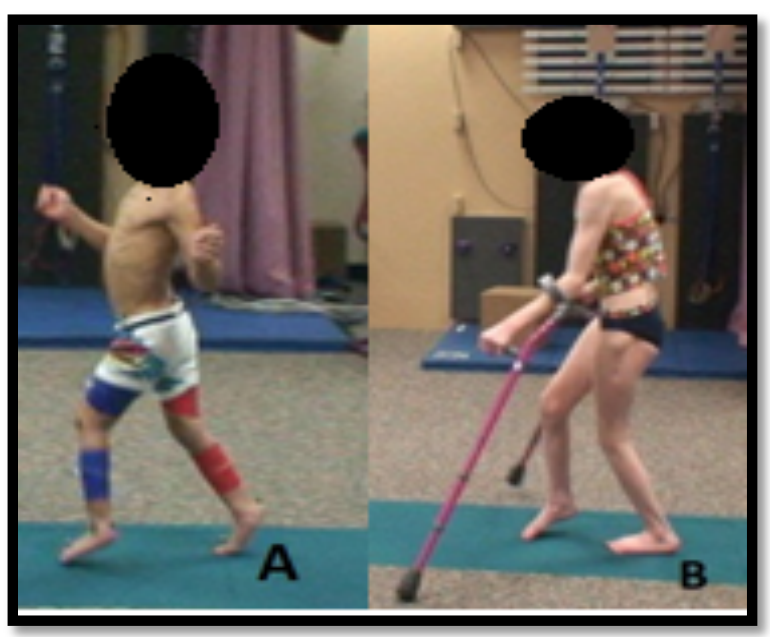

Figure 37 Children with CP in (A) Equinus and (B) Crouch Gait Deformity.

While there is a great deal of heterogeneity in children with $\mathrm{CP}$, two gait patterns are most commonly seen: equinus and crouch gaits. An equinus gait is characterized by the 
individual's ankle being plantar flexed (on one's toes) at floor contact (Figure $37 \mathrm{~A}$ ). In a crouch gait, the knee is excessively flexed in both swing and stance (Figure $37 \mathrm{~B}$ ).

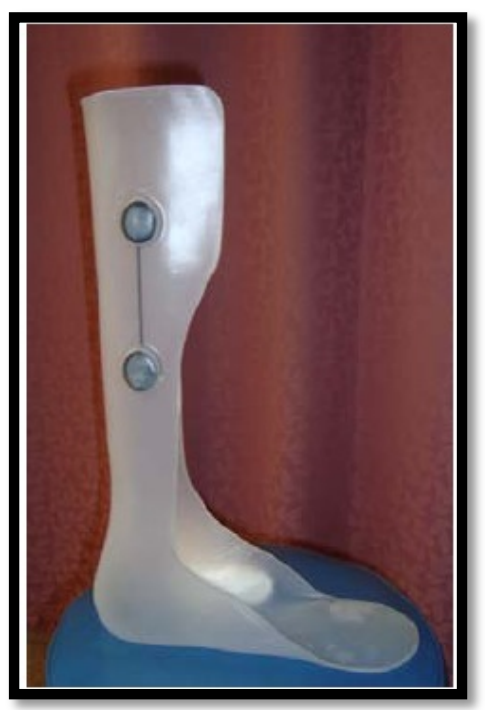

Figure 38 Ankle Foot Orthosis (AFO)

AFOs (Figure 38) are often prescribed for both gaits to aid in their walking. The large number of individuals with $\mathrm{CP}$ that use AFOs highlights the magnitude of the problem. United Cerebral Palsy reports that an estimated 764,000 people in the United States have one or more symptoms of $\mathrm{CP}$, and more than $50 \%$ of these individuals are prescribed orthoses [70][71]. When a child has an equinus gait, the AFO promotes heel contact by maintaining the ankle in a more neutral position. Having the plantar flexors of the ankle held stretch by the AFO may help also prevent muscle contractures by putting muscles in a lengthened position, and preventing deformity by controlling the position of the foot/ankle. If a child has a crouch gait, an AFO can reduce knee flexion in stance. In this case, the AFO provides a moment at the ankle that resists ankle dorsiflexion in stance. 
These findings are illustrated in Figure 39 and Figure 40, which show data collected from the Vicon ${ }^{\circledR}$ system on one of the pilot study's CP children. In Figure 39, the ankle joint angle range is greatly limited by the AFO. In Figure 40, the AFO helps to promote a heel strike, as the gait without the AFO just has a 'dip' in the shank angular velocity after terminal swing, suggesting a flat-foot stomping on the ground or a forefoot landing.

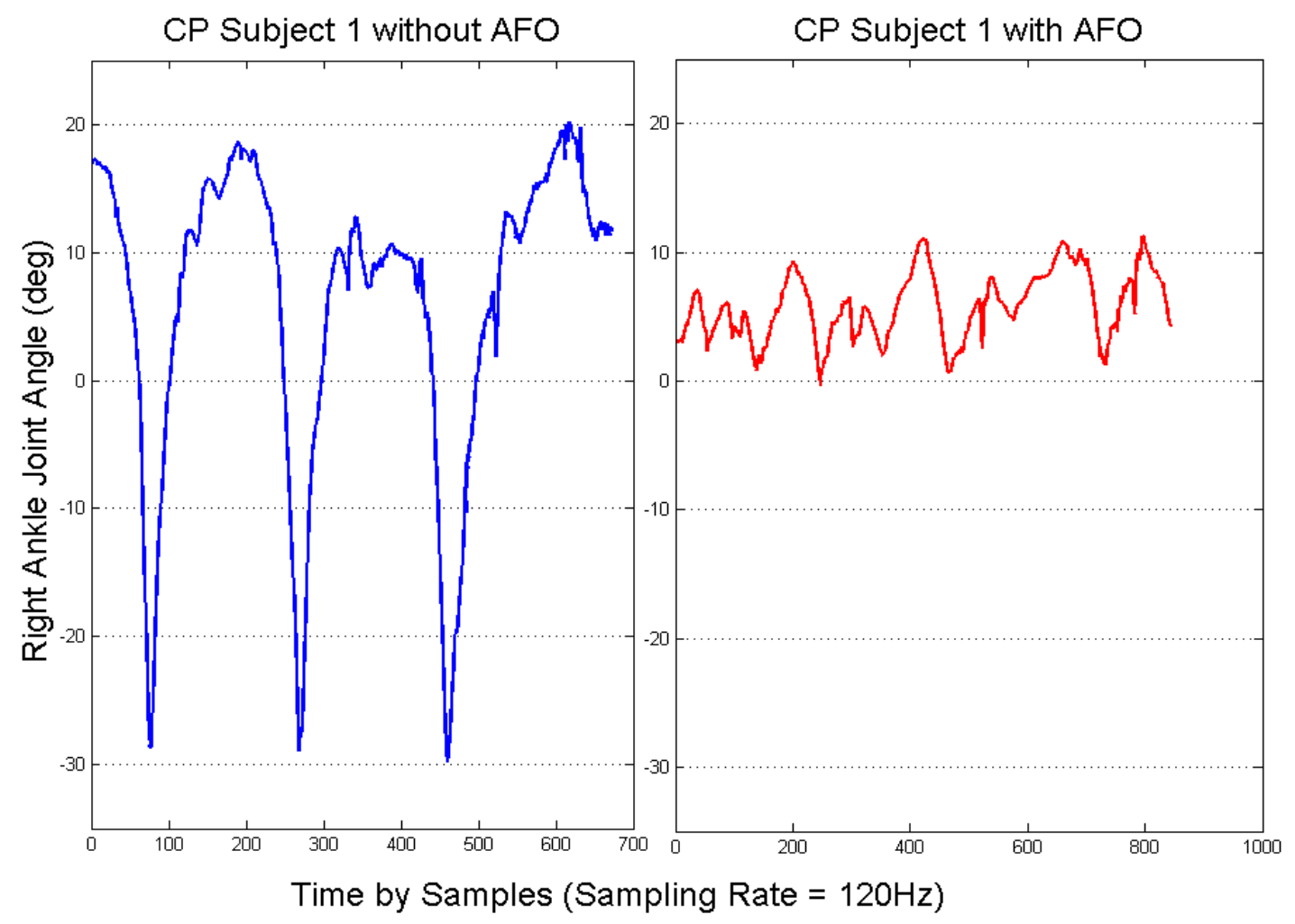

Figure 39 Ankle Joint Angle of CP Gait without and with AFO - the AFO Limits the Range of Ankle Motion 


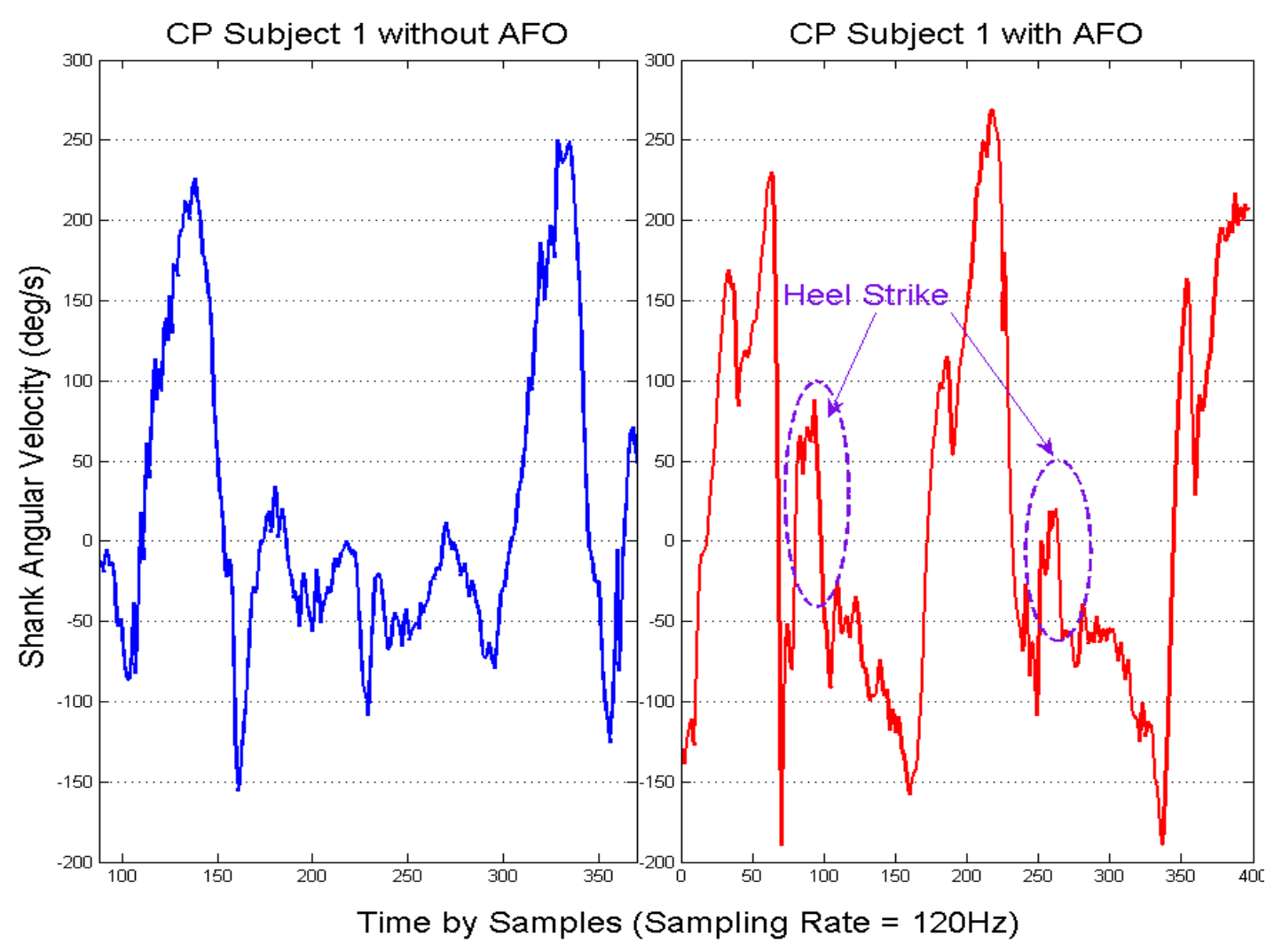

Figure 40 Shank Angular Velocity of CP gait without and with AFO - the AFO Helps to Promote a Heel Strike

In general, the prescription of AFOs usually has several treatment goals, including: (1) facilitating walking by controlling the position of the ankle and providing a base of support, (2) preventing contractures by putting muscles in a lengthened position and providing variable ranges of motion, and (3) preventing deformity by controlling the position of the foot/ankle. Although laboratory tests - usually gait analysis of patients with $\mathrm{CP}$ wearing $\mathrm{AFOs}$ walking in clinics or camera-instrumented motion capture laboratories - provide some insight into the first goal, results in naturalistic settings are lacking and may be significantly different. Moreover, without more continuous, longitudinal measurement to help understand the long-term effects of AFO use (i.e. how 
much it improves gait and how much it is worn in daily life in a longitudinal scale), the efficacy of AFOs in achieving the second and third goals cannot be determined. To better understand how AFOs perform in aiding individuals with walking disabilities and to further enhance their efficacy, longitudinal, continuous, non-invasive measurement is necessary.

\subsubsection{Hypothesis}

Inertial body sensor networks (BSNs) represent a promising platform for addressing the need mention in 6.1.1. [72], [73] and [74] are just a few of the papers that have demonstrated the potential of using BSNs for assessing gait parameters including knee and ankle joint angles. With MEMS sensor technology, ultra-low power processing and wireless communication, and high flash memory densities to reduce transmissions, the form factor and battery life of these sensor nodes minimizes invasiveness and enhances ease of use.

It is generally believed that a crucial factor in the development of contractures is the angular position of a joint as a function of time. The measurement of joint angle also allows determination of dorsi-/plantar-flexion mode (Figure 41), range of ankle motion, and ankle joint angular velocity. Therefore, continuous monitoring of the ankle joint angle for children with $\mathrm{CP}$ wearing $\mathrm{AFOs}$ is the ideal method to evaluate the

effectiveness of the AFOs to prevent contractures. This information obtained would affect both the prescription of AFOs to address contractures as well as the construction of the AFOs themselves (e.g., the joint angles it maintains in the ankle). 


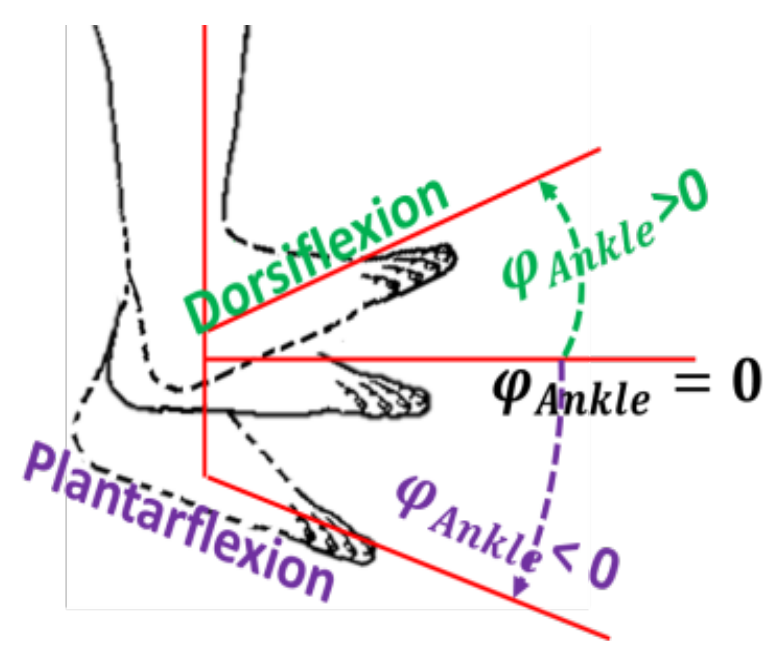

Figure 41 Dorsiflexion and Plantarflexion

However, while these sensors provide rich information on the kinematics of human motion, the accurate spatial information required for many gait parameters is challenging to obtain, especially for pathological gait. Intrinsic sensor noise causes integration drift, the uncertainty of mounting nodes on human bodies causes a systematic bias, and movement in multiple planes causes computational errors, all of which hinder the acquisition of accurate joint angles and other spatial features. These challenges must be addressed first in order to achieve accuracy that suffices the application requirement.

\subsubsection{Experiment Setup}

\subsubsection{Test on a Healthy Subject}

Since walking for children with $\mathrm{CP}$ is a challenging task, we could not afford fumbling through a data collection with children with $\mathrm{CP}$. To make sure that the experiment is well designed and the hypothesis is valid, we recruited a healthy subject to simulate the experiment for a pilot study. This healthy subject had TEMPO devices mounted on his 
shanks on the AFOs with mounting tape, and tightened on the shoes (forefoot) with elastic Velcro ${ }^{\circledR}$ straps, as shown in Figure 42.
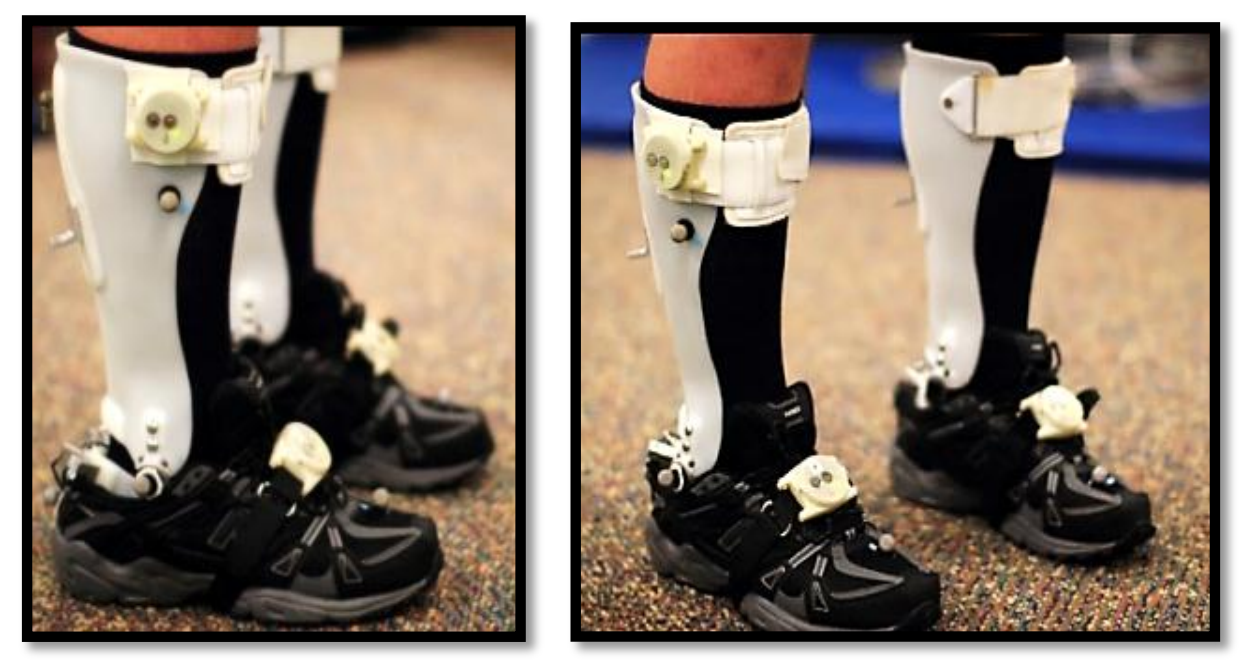

Figure 42 Healthy Subject Wearing AFO for Experiment

First, the subject was asked to stand still on the treadmill for 10 seconds and then to swing his legs as a signature motion in the beginning for the synchronization between two systems. Next, the subject was asked to walk normally and to simulate crouch gait and equinus gait on the treadmill at a speed of $1.6 \mathrm{~km} / \mathrm{h}$. Each of the walking patterns was conducted for one minute. In the second set, the same subject was fitted with nonarticulated AFOs. The subject repeated the same procedure as the first set of experiments, conducting each of the walking trials on the treadmill for one minute. All of the kinematic data was collected and saved in a computer located in the gait lab and postprocessed in Matlab®.

The ankle joint angles obtained from these experiments are plotted in Figure $43 \sim$ Figure 45 , together with the validation from Vicon ${ }^{\circledR}$. The ankle angle calculated by TEMPO 
system has an average RMSE of 2.05 degrees as compared to Vicon $®$. Since the error is within the range of the error margin of the reference system (about 3 degrees), it is more important to focus on whether or not the computed ankle joint angle can be interpreted with similar information about $\mathrm{CP}$ gait as the reference system. For example, the range of the ankle angle and the time percentage of ankle spent in dorsi-/plantar-flexion are interesting parameters to be derived from ankle joint angle.

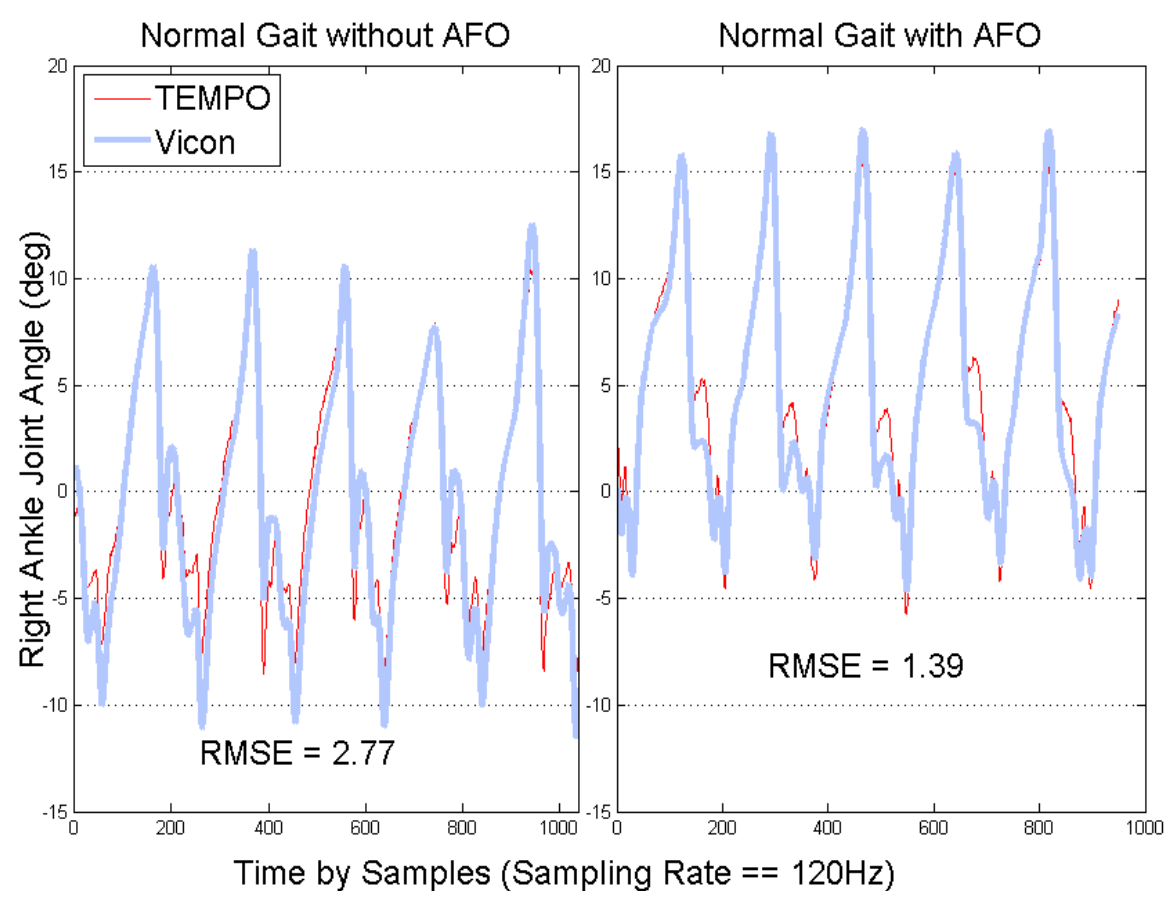

Figure 43 Ankle Joint Angle of Normal Gait 


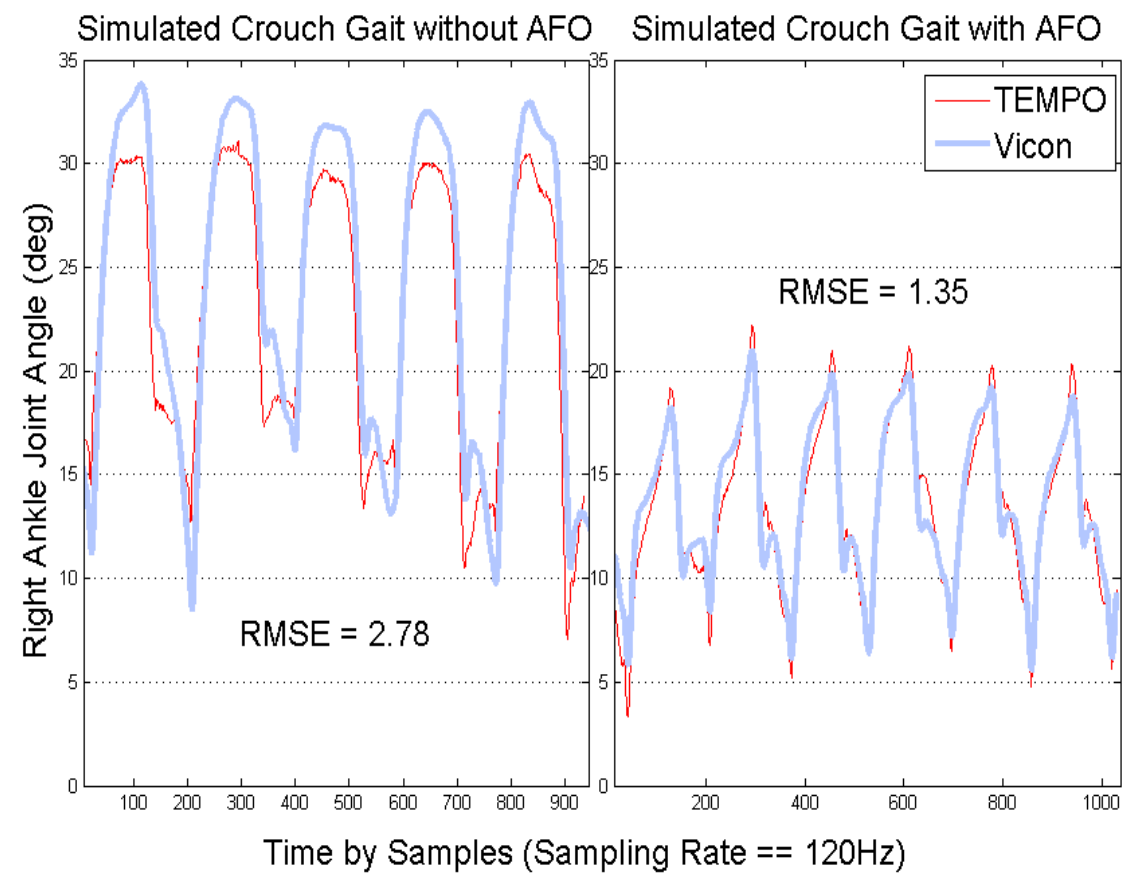

Figure 44 Ankle Joint Angle of Simulated Crouch Gait

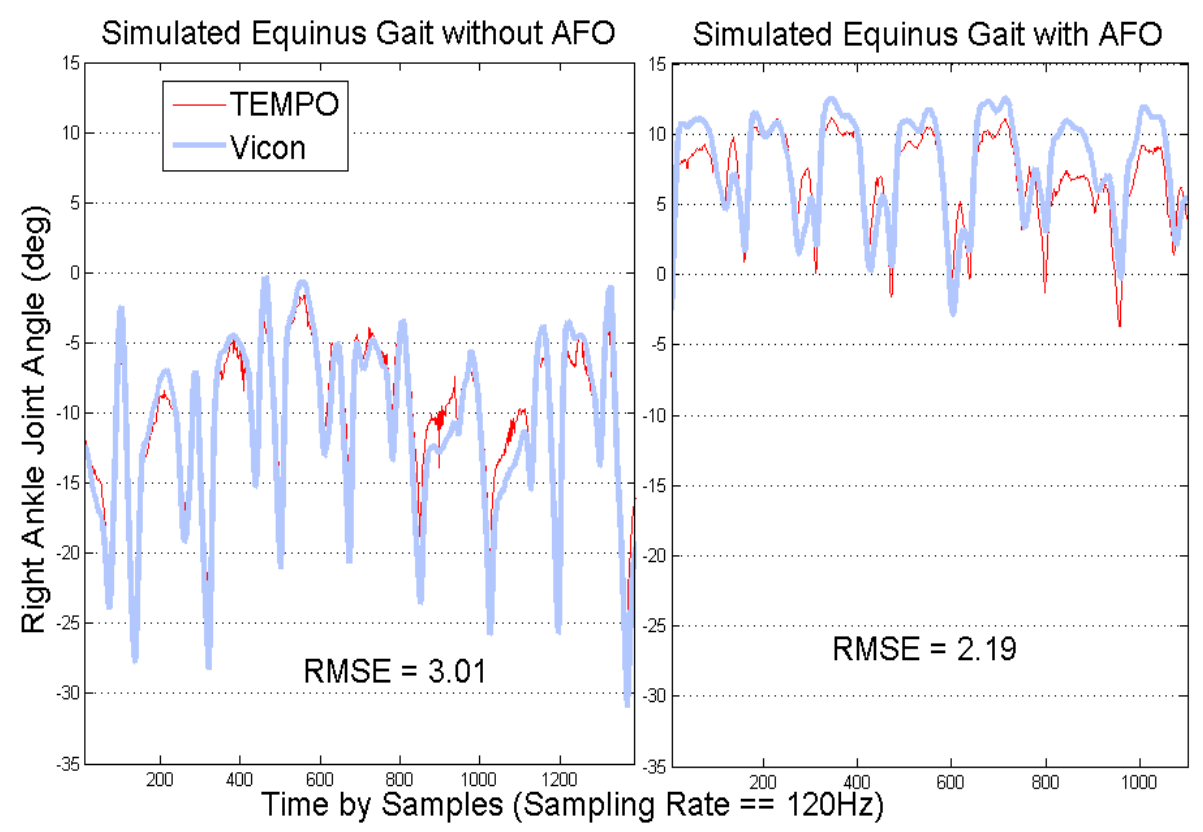

Figure 45 Ankle Joint Angle of Simulated Equinus Gait 
One of the major errors comes from the difference in foot models between the inertial sensor system and the optical capture system. The foot angle computed by the inertial sensor system has the most discrepancy during the stance phase after the heel-strike. The reference system records a continuous increase of foot angle as the foot is pushing down on the ground; however, the inertial system records a foot signal decreasing as if the foot is lifting up. Because the motion during the stance phase does not affect the range of ankle motion nearly as much as the motion during the swing phase, this error does not greatly affect the ankle motion analysis for this application.

Observations from the three sets of data from the comparison study on the healthy subject illustrate the impact of AFO on ankle joint angle during a short period. As stated in the previous medical research, AFOs tend to limit ankle motion range, especially during plantar-flexion. For the simulated crouch gait, the AFO eliminates some of the excessive dorsiflexion but does not at all help to generate plantar-flexion. For the simulated equinus gait, the AFO decreases the excessive plantar-flexion and increases the dorsiflexion to a normal level.

\subsubsection{Experiment in Children with $\mathrm{CP}$}

Having established the methodology through initial experiments on healthy subject, we apply the approach to children with $\mathrm{CP}$ as part of a pilot project to collect data and extract ankle joint angle in the Vicon ${ }^{\circledR}$ instrumented lab. Data has been collected on four CP subjects wearing AFOs. These subjects walked on the ground within the range of the Vicon ${ }^{\circledR}$ cameras for several trials (about 5 meters each trial). Subject consent and assent 
was approved by the Institutional Review Board (IRB) at the University of Virginia and obtained for all subjects.

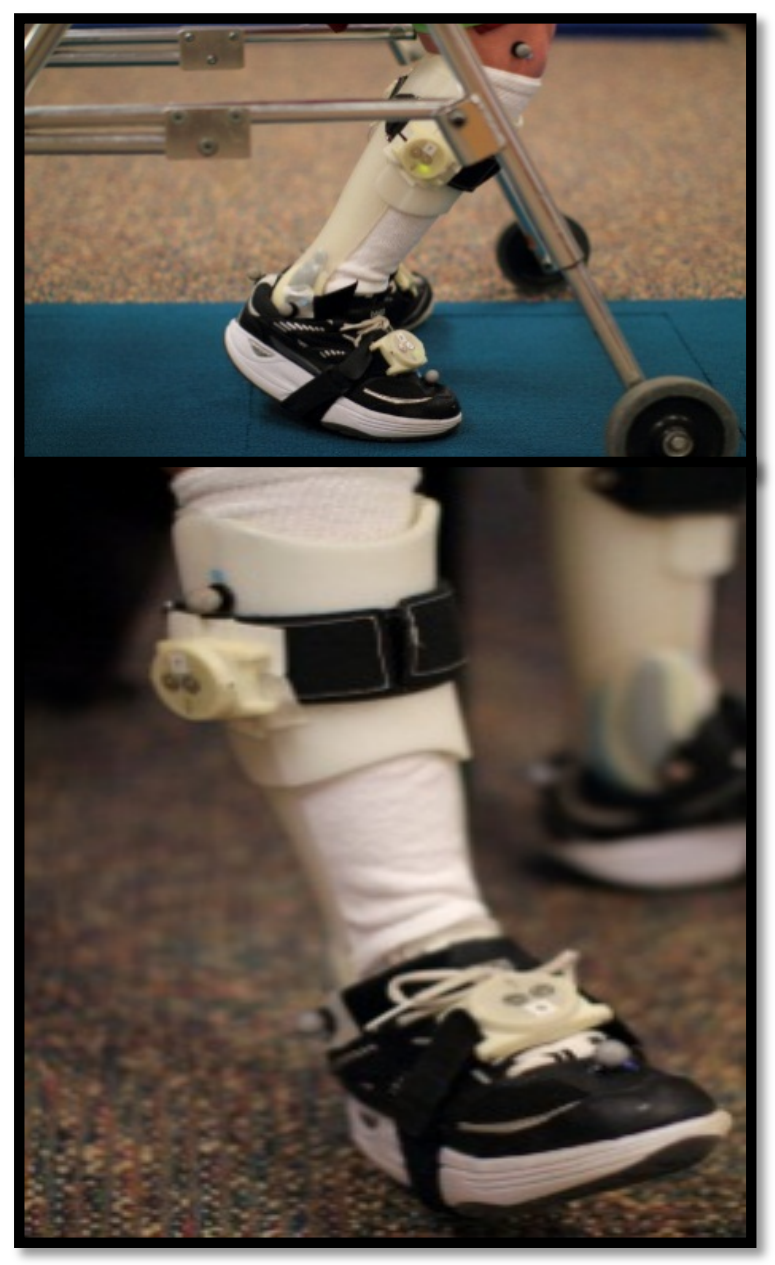

Figure 46 Children with CP Instrumented with Inertial BSN Nodes for Experiment

Some walking aid devices (walkers) block the line-of-sight of optical motion capture system, challenging the validation system to provide continuous monitoring. Mounting calibration can also be challenging since the required knowledge (initial sement angle) is 
difficult to obtain from $\mathrm{CP}$ subjects with crouch gait as they cannot stand straight as a healthy subject can.

Therefore,with the assistance from the research staff, the subjects' shanks were held straight for 5 seconds in order to proceed with the mounting calibration. The initial swing motion for synchronization between the Vicon ${ }^{\circledR}$ system and TEMPO system was also avoided since it was almost impossible for the CP subject. Instead, the finer offline synchronization is done by using the first shank motion of the walking trial and aligning shank and foot angle signals and foot angle signals from TEMPO with the validation system.

\subsubsection{Ankle Joint Angle Extraction}

Ankle joint angles versus time for the four CP subjects are plotted in Figure 47. With an average RMSE of 2.41 degrees, it is evident that the TEMPO inertial BSN system can track the ankle joint angle in time for CP subjects, showing the potential for longitudinal study. 

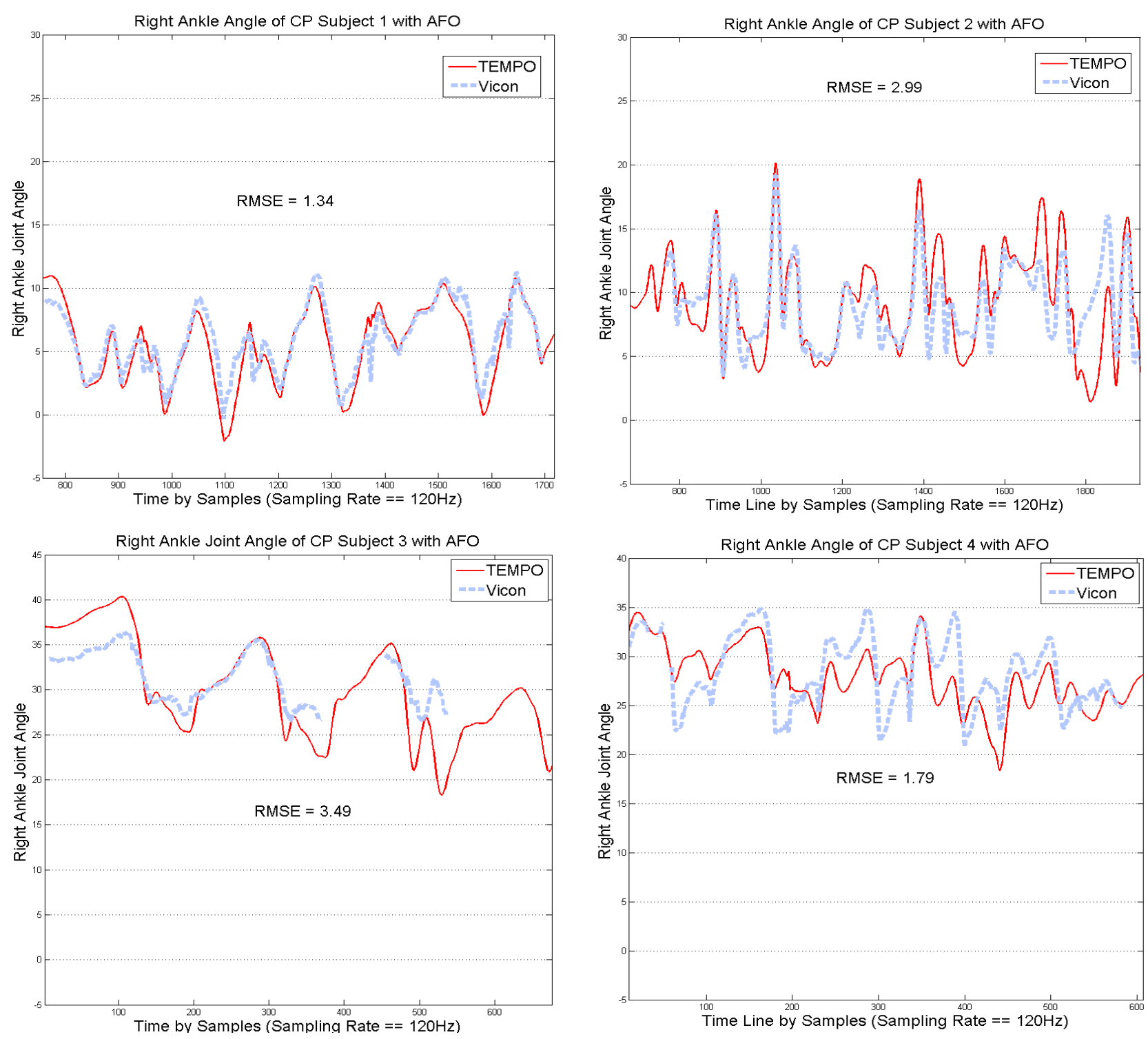

Figure 47 Ankle Joint Angle of Children with CP Wearing AFO

\subsubsection{Other Gait Parameters for Assessing Ankle Foot Orthosis}

Other established parameters for CP gait assessment are also extracted on a per gait cycle basis. While a detailed time series of ankle joint angle could indicate the pathologies in certain gait patterns, features from the time series can provide the first impression in AFO efficacy in daily use and effectively reduce data to be stored. For example, cadence (steps/min) is a parameter of interest and can be considered as temporal information, 
which can be obtained accurately and precisely at the sampling rate no less than $120 \mathrm{~Hz}$ by recognizing gait cycle patterns as detailed in Chapter 3.

\subsubsection{Ankle Angle Range}

Ankle motion range and percentage of dorsi-/plantar-flexion is another parameter of potential interest. The ankle motion range is the difference between the maximum ankle joint angle and the minimum ankle joint angle in one gait cycle. It can be obtained after extracting ankle joint angle and segmenting gait cycles. This parameter can also indicate the percentage of ankle motion that is in dorsiflexion mode (ankle joint angle $>0$ ) or plantarflexion mode (ankle joint angle $<0$ ) per gait cycle. In future work, features such as ankle joint angle range and percentage in dorsi-/plantar-flexion are necessary for data reduction in the longitudinal data analysis.

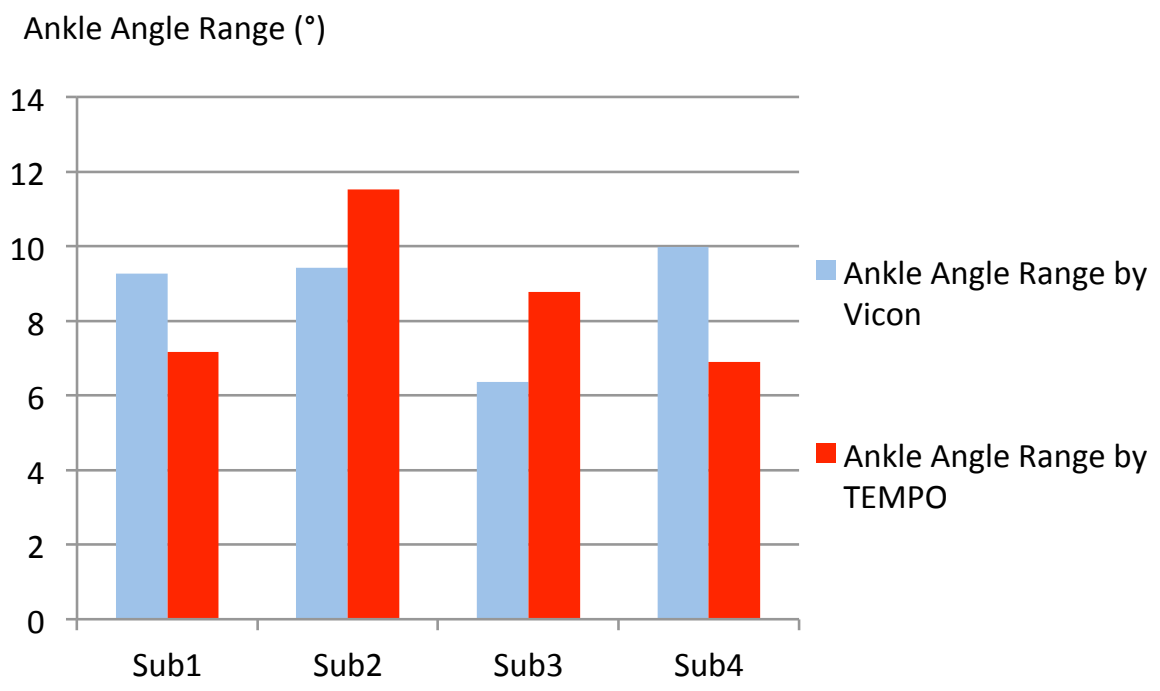

Figure 48 Average Ankle Angle Range per gait cycle of 4 CP subjects with AFO 


\subsubsection{Cadence}

Cadence (steps/min) is another parameter of interest and can be considered as temporal information. It can be obtained accurately and precisely at the sampling rate of $120 \mathrm{~Hz}$ by recognizing gait cycle patterns. With an $\mathrm{AFO}, \mathrm{CP}$ subject 1 shows an increment in cadence of $82.8 / \mathrm{min}$ comparing to $74.4 / \mathrm{min}$ without AFO. Similar results (i.e., increased cadence when wearing AFOs) were also found in the simulated CP gait.

\subsubsection{Gait Complexity}

Figure 49 and Figure 50 show the shank phase portrait difference between a healthy subject and a CP subject. Comparing to the phase portrait of the healthy subject, the phase portrait of the $\mathrm{CP}$ subject does not converge to an attractor because the irregular shank motion cannot form the cyclic pattern as the normal healthy subject. This discovery motivates quantification of gait complexity for assessing the efficacy of AFOs. 


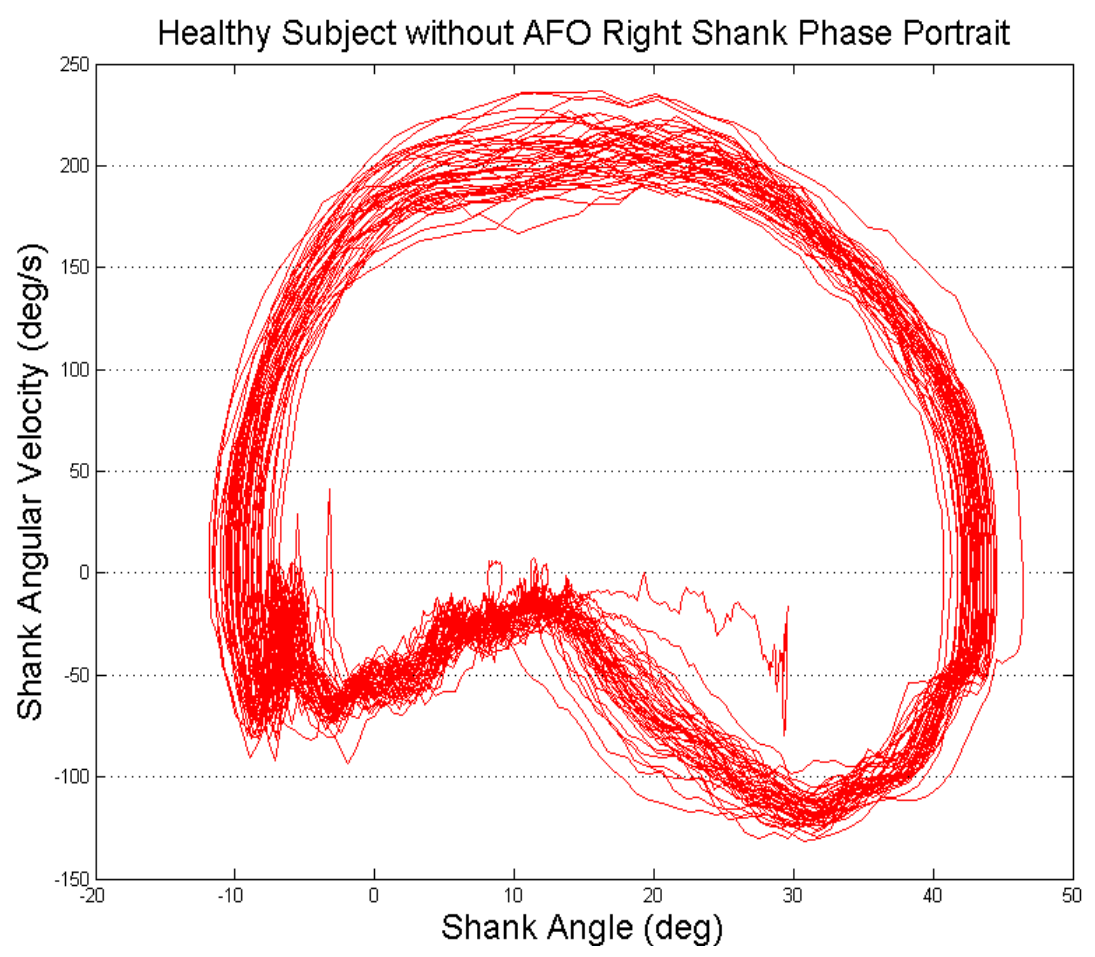

Figure 49 Health Subject Shank Phase Portrait without AFO

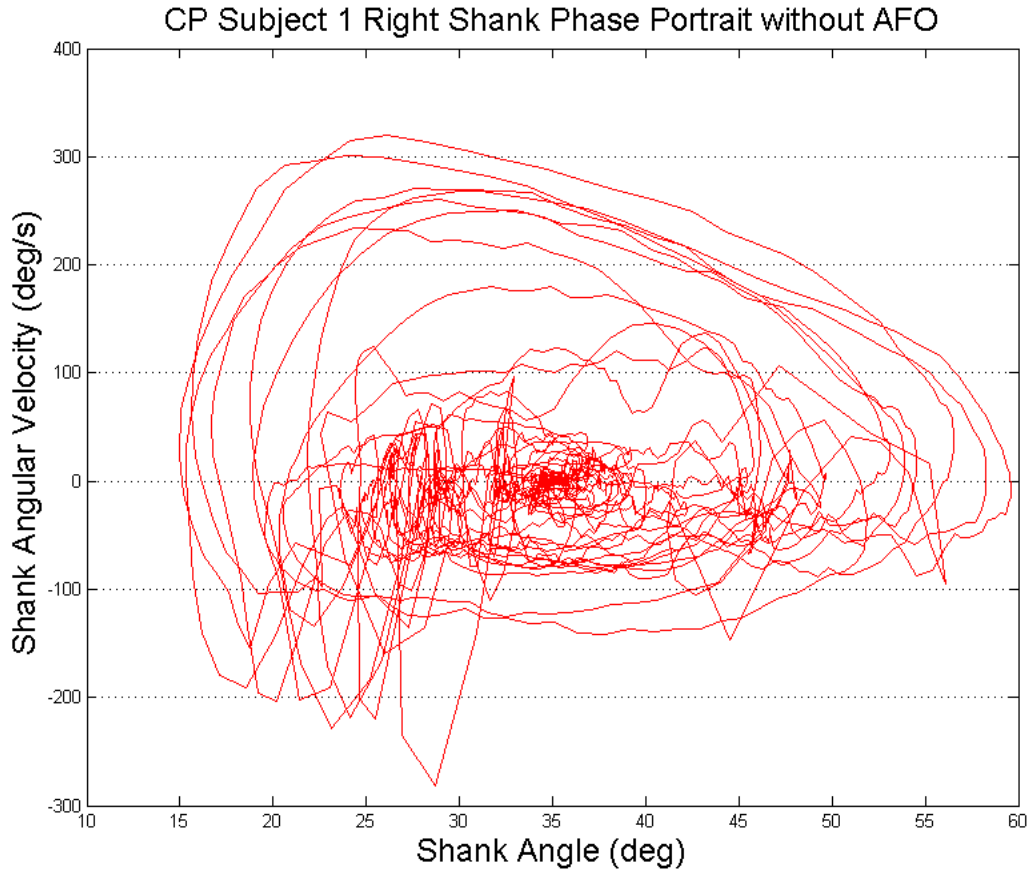

Figure 50 Shank Phase Portrait of CP Subject without AFO 
Gait complexity parameter can be extracted based on the approach detailed in [51]. It is gauged by number of elliptical Fourier curves needed to approximate a phase portrait. The more elliptical Fourier curves are needed to approximate a phase portrait of a gait signal, the more complex the gait signal is. By applying the gait complexity parameter, we obtain the quantification of gait complexity as shown in Figure 51.

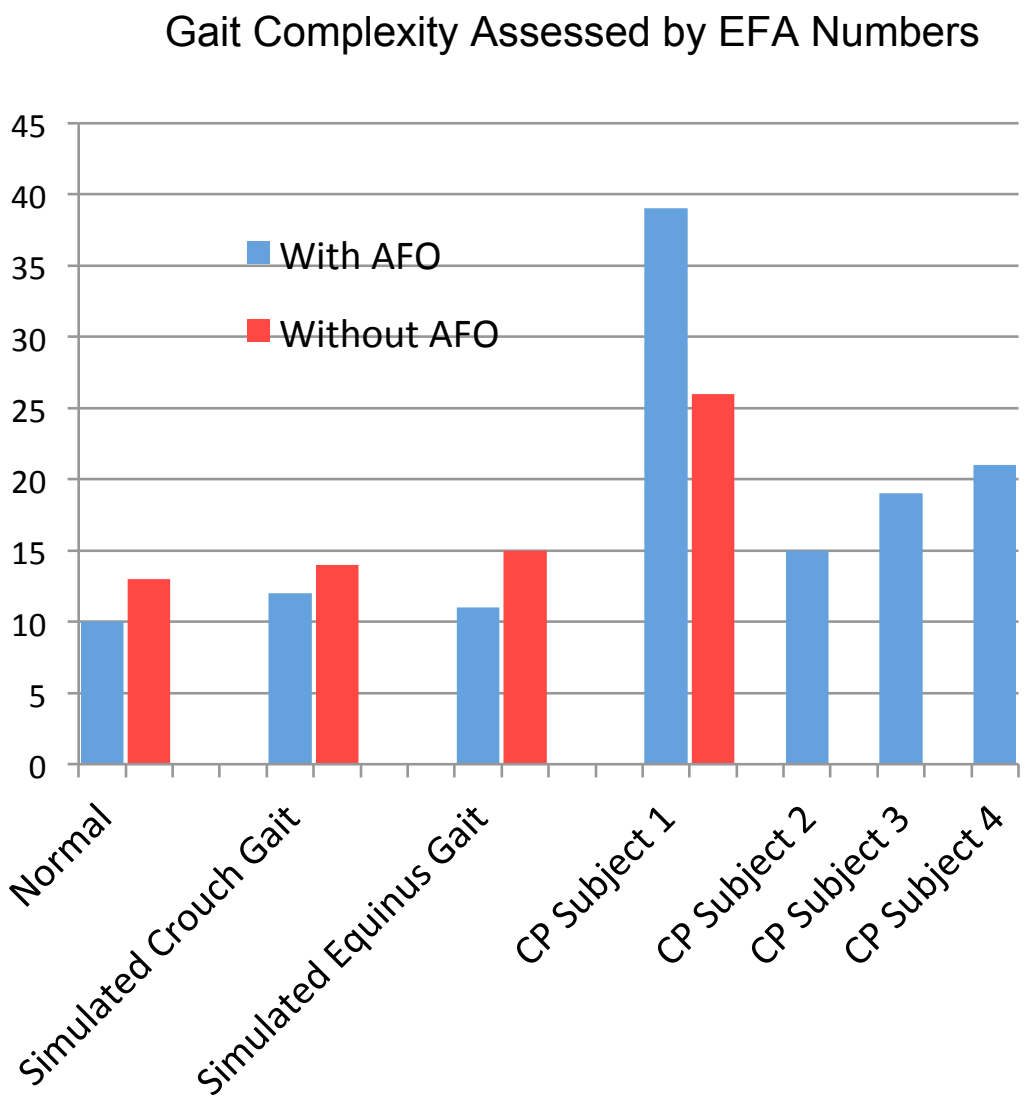

Figure 51 Gait Complexity Assessment of CP Gait 


\subsubsection{Discussion}

In summary, in addition to providing validation of the methodology presented in Section 6.1.4, the gait parameters analysis above provides insight into how AFOs affect ankle motion. For example, Figure 44 and Figure 45 reinforce the general conclusion drawn from ankle joint angle signal that the AFO is helpful in limiting plantarflexion. However, for the $\mathrm{CP}$ subjects in crouch gait, AFOs are not designed to limit the excessive dorsiflexion during stance.

The different gait pathologies from the pilot study have shown the necessity of specific AFO designs and tailoring for each individual's pathological $\mathrm{CP}$ gait. The results achieved in this case study suggest that our methodology can be successfully applied for this purpose and be effective across a variety of gaits pathologies. The ability to collect longitudinal data will enhance our understanding of the effects of AFOs and their ability to improve the gait of children with CP in real life situations. In addition, it will allow us to evaluate the ability of these devices to identify if and how AFOs can prevent contractures at the ankle.

\subsection{Aiding Diagnosis of Normal Pressure}

\section{Hydrocephalus}

\subsubsection{Medical Background}

Normal Pressure Hydrocephalus (NPH) is a neurological condition caused by abnormal accumulation of cerebrospinal fluid (CSF) around the brain [75]. Although NPH can 
occur in any age, it is normally observed in the aging group. According to recent population-based studies, the prevalence of NPH to be about $0.5 \%$ in those over 65 years old, with an incidence of about 5.5 patients per 100,000 of people per year [76]. The symptoms of NPH are usually described as a classic triad of gait disturbance, dementia or mental decline, and urinary incontinence [75], with debilitating effects on patients' and families' quality of life. However, due to the similarities of these symptoms to many aging disorders, such as Alzheimer's disease and other forms of dementia, and individual variability of the symptom expression, the differential diagnosis is challenging. In comparison to other forms of dementia, NPH is reversible after proper medical intervention and may significantly improve patients' and caregiver's quality of life. If diagnosed successfully as NPH, patients will be treated with an invasive, long-term intervention - the ventriculo-peritoneal (VP) cerebral shunt to drain excess CSF to the abdomen where it is absorbed. This shunt surgery does however present great risks, in that it may cause hematoma, cerebral edema, crushed brain tissue and herniation. Therefore, the accuracy of NPH diagnosis is critical.

\subsubsection{Hypothesis}

To diagnose NPH, a high volume lumbar puncture (HVLP) is performed to remove excess fluid from a suspected NPH patient followed by a clinical evaluation of the cognitive and functional response (Figure 52) [75]. Medical literature has suggested that improvement in gait pre- to post-HVLP is often a good functional marker for diagnosis in the decision to proceed with shunt surgery [75]. However, in current clinics, visual observation is still the dominant form of assessment for gait performance; thus, only 
simple gait features such as walking speed (based on time to walk 10m) and average stride length/time (based on the number of steps to walk $10 \mathrm{~m}$ ) are used. The low resolution and precision of such features may hinder observing separability in gait performance pre- and post HVLP.

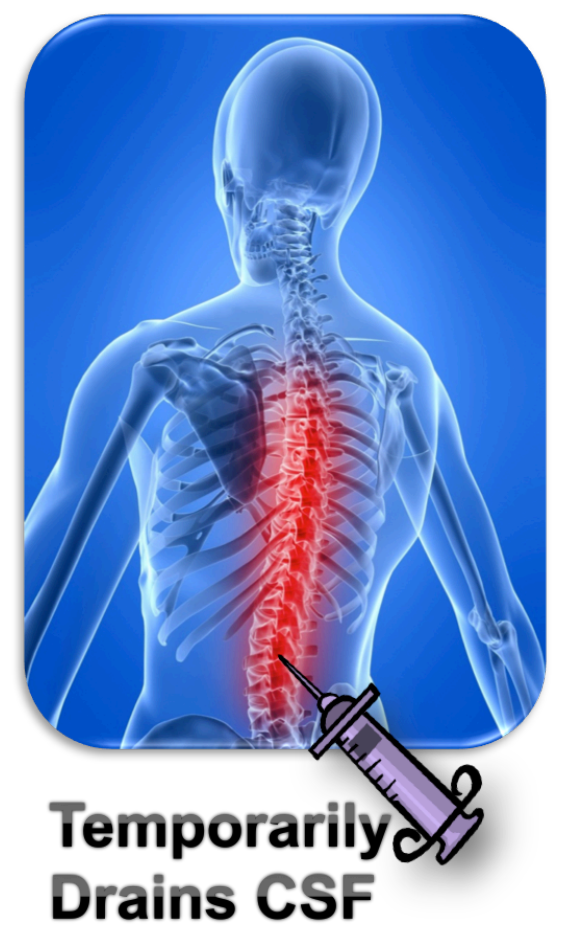

\section{Before HVLP} Brain imaging Cognitive skills assessments Gait performance

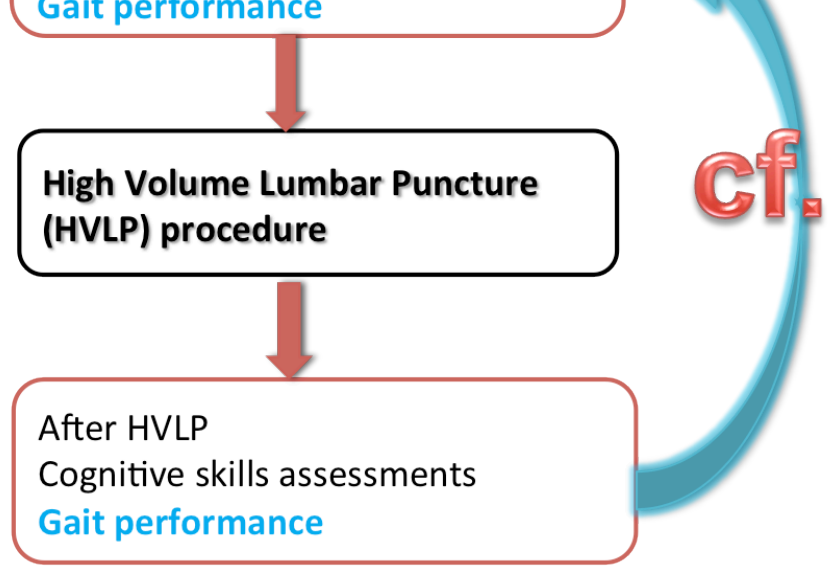
Figure 52 Differential Diagnosis of NPH in Clinics

Some clinics have adopted advanced optical motion capture systems for higher precision gait analysis. However, that not only requires a dedicated laboratory with expert operation but also involves a cumbersome setup procedure that makes it impractical for use on impaired, fatigued patients who are already undergoing significantly invasive procedures throughout the day [77][78]. This is especially problematic given that patients have variable HVLP response times, thus longer continuous gait monitoring is necessary 
to provide high confidence NPH diagnosis. Therefore, measurement tools that can provide rich, high precision gait parameters with the longitudinal monitoring potential are impactful to the diagnosis.

Inertial BSNs may be the promising platform for addressing this need. The rich information provided by $6+$ degrees of freedom motion capture systems has enabled many medical studies and clinical applications [60][79][80][81]. However, in order to explore gait features that maximize separability for $\mathrm{NPH}$, as well as other differential diagnoses, several challenges must be overcome. First, gait events need to be accurately detected in order to extract the temporal features from the noisy inertial sensor data. Second, while inter-subject gait features may show great separability, the intra-subject gait difference before and after HVLP may be significantly subtler. Third, the variance in the collected data could also be reflecting the noise in neuromuscular control due to the different times of the day, environments, energy levels, or even moods of the patient. In other words, one could naturally (voluntarily or otherwise) change certain gait parameters instead of responding to HVLP. Fourth, new quantitative metrics must be established to assess certain gait characteristics that were assessed by visual observation previously.

To address these challenges and to explore the feasibility of extracting and leveraging advanced BSN-enabled gait features for enhanced separability in NPH diagnosis, a pilot study was performed on six suspected NPH patients with BSN data collection added to the gait assessment protocols before and after HVLP. To capture intra-personal NPH gait symptoms, methods for personalized signal processing - looking for changes in an individual's gait pre- to post-HVLP rather than extracting absolute measures - are 
designed. Then gait features were extracted from inertial BSNs and compared before and after HVLP in order to evaluate the gait improvement and to identify gait features providing separability for NPH diagnosis.

\subsubsection{Experiment Setup}

Data was collected on six suspected NPH patients pre- and post-HVLP. Subject consent and assent was approved by the University of Virginia's IRB and obtained for all patients. Existing methods for cognitive and functional assessment were used, but TEMPO data collection was added to the 10-meter walk. TEMPO nodes were placed on both wrists, both shanks and the waist, as shown in Figure 53. The inertial sensor data was streamed via Bluetooth ${ }^{\circledR}$ to a portable laptop carried by a technician following the patients. All data collected was post-processed and analyzed in Matlab®.

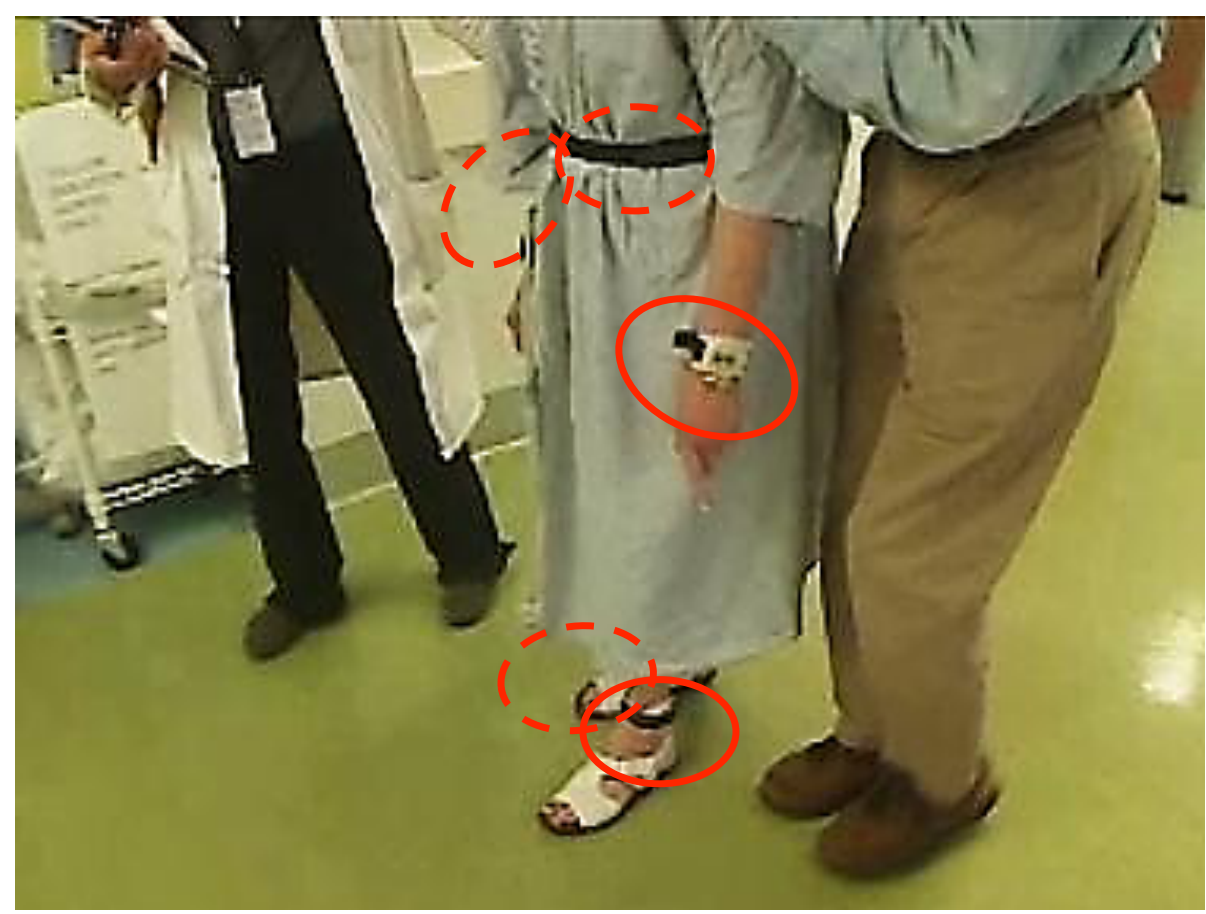

Figure 53 Suspected NPH Subjects Mounted with TEMPO Inertial BSN Nodes 
A neurosurgeon inspected the suspected patients after their cognitive and functional assessments were completed pre- and post-HVLP, and decisions were made about prescribing the VP shunt or ruling out NPH. Due to the risk of missing possible NPH patients whose quality of life could be dramatically improved through a VP shunt, neurosurgeons tend to err on the side of shunting borderline cases, which presumably results in a low number of false negative diagnoses. (Note: the number of false negatives cannot be determined.) However, patient follow-up is necessary to identify any false positive diagnoses, which would be apparent from lack of a positive response to the VP shunt placement. According to the follow-up information for this study, the four patients diagnosed as NPH have regained non-negligible cognitive and functional ability after the shunt surgery, confirming the correctness of the diagnosis (i.e., true positives).

\subsubsection{Features Extracted from Clinical Measurement}

Prevailing clinical practice for evaluating ambulation function is still based on subjective clinical observation. More objective assessments usually adopt a stopwatch-timed 10meter walk with a step count. The setup is easy to deploy in clinics and provides gait features such as gait speed and average step length and time. The results from the pilot study are shown in Figure 54. 

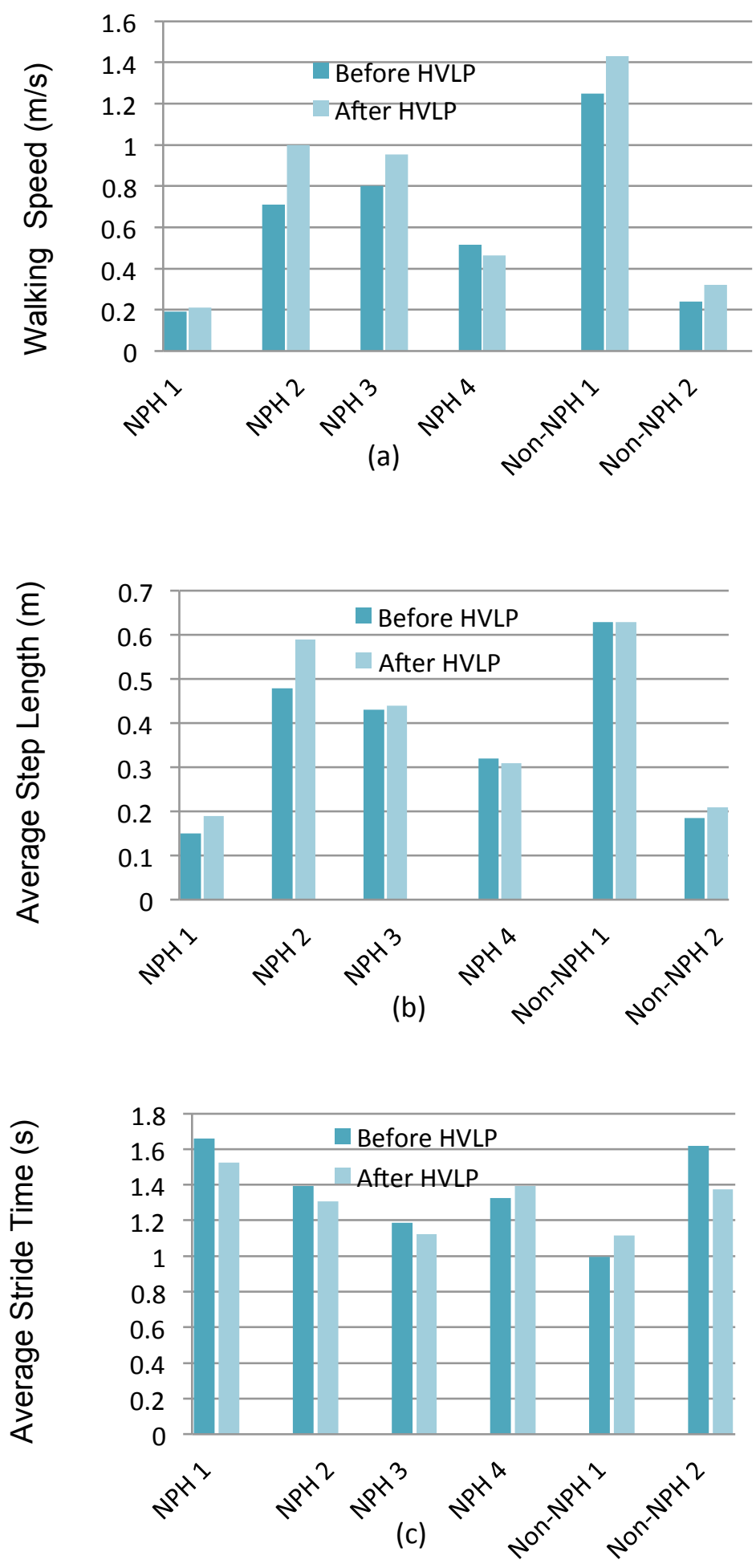

Figure 54 Clinical Obtainable Gait Features (a) Gait Speed (b) Step Length and (c) Stride Time Fail to Separate the Suspected NPH Group 
Figure 54 shows that the gait speed, average step length, and average stride time, respectively, of six patients (four of whom were later determined to have NPH) pre- and post-HVLP based on the timed 10 meter walk. These bar charts reveal that gait speed and step length do not always increase and stride time does not always decrease post-HVLP, indicating that these parameters do not provide good separability for gait-based NPH diagnosis. This finding motivates the exploration of extracting other gait features for separating the NPH patients from the non-NPH patients, including features that cannot be extracted from visual observation alone. We move on to finding the features from inertial BSN data that would have provided that separability by identifying new gait features.

\subsubsection{Features Extracted from Inertial BSNs}

\subsubsection{Stride Time Standard Deviation}

Stride time variability is sometimes associated with gait stability [82]. Although average stride time can be obtained with a stopwatch and step counting, it does not provide information about cycle-to-cycle stride time. Greater accuracy, precision, and runtime of stride time assessment may be obtained by segmenting gait cycles. In this study, a peak detection algorithm is used to find the highest peak in the z-plane (sagittal) gyroscope signal (maximum shank angular velocity shown in Figure 6), which identifies the midswing event. To suppress the ripples in the gyroscope signal, a zero-phase, 3rd order, Butterworth low-pass filter with an empirically determined cutoff frequency of $3 \mathrm{~Hz}$ is used, preventing the peak detection algorithm from selecting the random peaks due to ripples. After the gait cycles are extracted, the standard deviation of stride time is computed based upon the stride data obtained. 


\subsubsection{Double Stance Time}

[83] has reported significantly reduced average double support time in the NPH group after shunt surgery. Detailed in Chapter 3, Section 3.1.3.4, double stance time is the duration when both feet are in contact with the ground. It can be counted as time from the heel-strike instance of one shank to the toe-off instance of the other shank. With the gait event detection methods detailed as in Section $\mathrm{xx}$, the double stance time can be counted.

\subsubsection{Lyapunov Exponent}

It is reported in [83] that the gait apraxia in NPH gait usually includes "poor balance, off balance, unsteady, wobbly, staggering, difficulty on stairs and curbs" with frequent falls. Since all these observations point to the instability of gait, we suspect that reversible abnormalities of NPH gait can be characterized by a measure of gait stability - an important feature for assessing walking balance and resulting fall risks [53][84].

To assess the stability of a dynamical system, nonlinear analysis tools have been adopted in biomechanics, such as the Poincaré map [49] and Floquet number [53]. However, it is argued in [54] that due to the variance that exists in human motion, orbital stability assessment based on the assumption that human motion is strictly periodic is not a good measure. Instead, for pseudo-periodic systems as human movement, local stability can be used to describe how the dynamic system responds to "very small perturbations continuously in real time" [54]. Lyapunov exponent (LyE) is such a parameter to quantify the local stability. 
To do so, first the phase portrait of the gait signal is reconstructed. [85] has demonstrated that inertial sensors are valid for such analysis by finding a high correlation (0.98) between inertial sensors and optical motion capture systems on the largest LyE. [57], [85] and [86] concluded that the inferior body segment is more sensitive to a small perturbation than the superior segment, and they found a larger change in the LyE of shank angular velocity than the thigh angular velocity. Therefore, for this study, shank signal is used to expect higher separability in gait stability assessment than the thigh placement. Shown on the top of Figure 55 is a healthy subject's phase portrait reconstructed from shank angular velocity, and on the bottom of Figure 55 are two figures of counterparts of NPH subject.

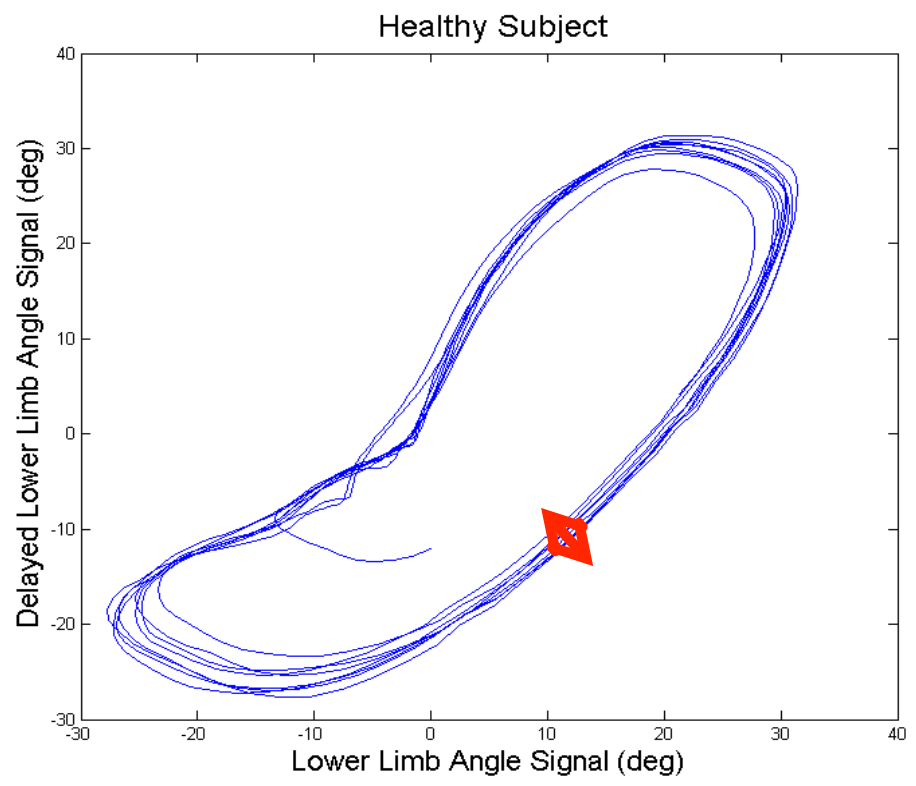



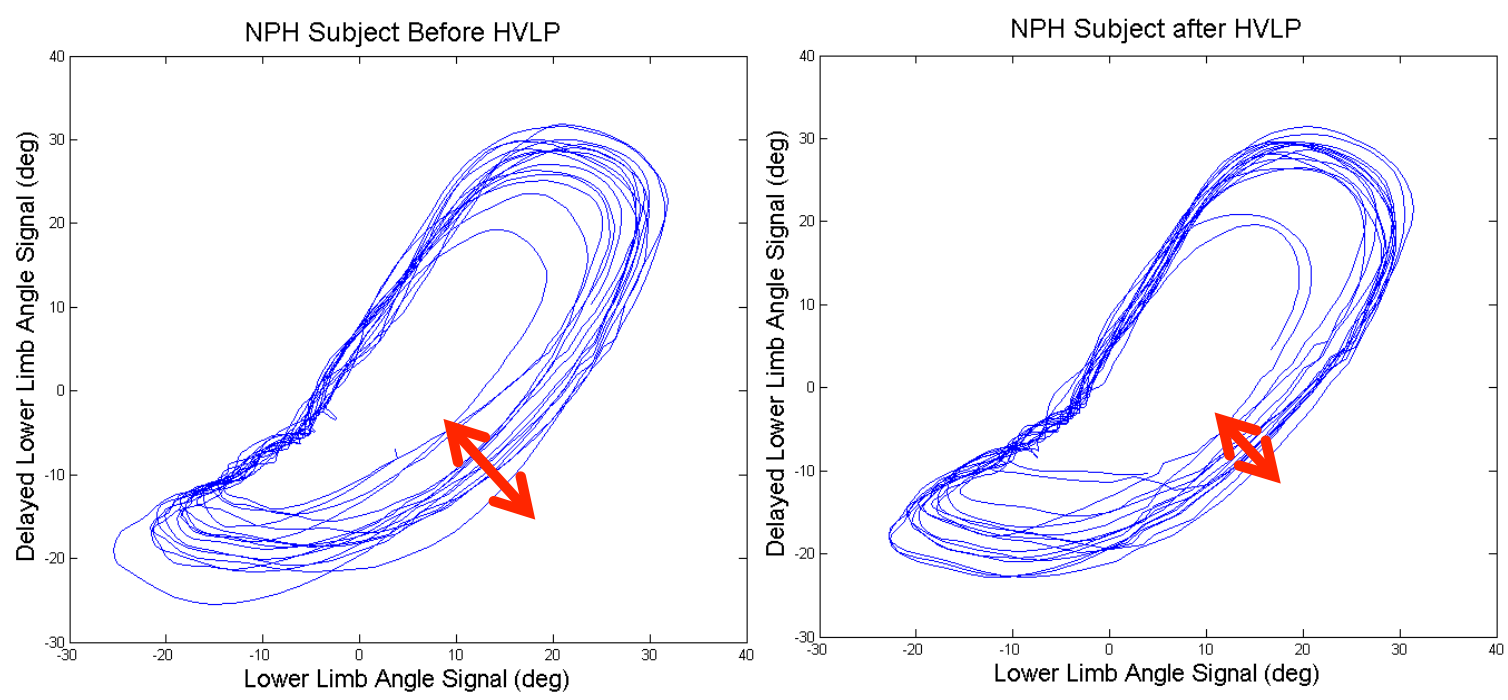

Figure 55 Reconstructed Phase Portraits of Healthy and NPH Subjects

The main difference between the top figure and the bottom two figures is that trajectories in the NPH subject's phase portrait diverge more quickly than that in the healthy subject's phase portrait. This is because the healthy subject's gait is more stable and it can form a more regular closed curve. Now we compare the NPH subject's gait after HVLP on the bottom two figures, and it seems less diverging compared to before HVLP. Of course, this is not as visually apparent as comparing to the healthy subject. LyE is exactly such a quantitative measure assessing the diverging rate between trajectories. The larger the LyE, the more quickly the trajectories diverges, and less stable the gait is. Thus higher positive LyE indicates lower gait stability.

After the phase space is reconstructed, LyE can be computed using either the Walgorithm [56]. To compute LyE for given time series data, the first two steps consist of finding the embedding dimension and time delay lag for phase space reconstruction. To find the embedding dimension of the time series, a False Nearest Neighbor (FNN) 
algorithm [55] is applied. With this algorithm, the time series of the shank's angular position is identified to have an embedding dimension of 2 . The time delay is determined by using average mutual information, and a time delay of 10 samples is used for computing LyE in this study. To eliminate the temporal variations in the signal, the shank angle of one gait cycle is normalized temporally with a period of 128 samples. The details for computing LyE is described in Chapter 4, Section 4.3.

\subsubsection{Results}

The results of the methods described from section 6.2.5.1, 6.2.5.2 and 6.2.5.3 are shown from Figure 56 to Figure 58.

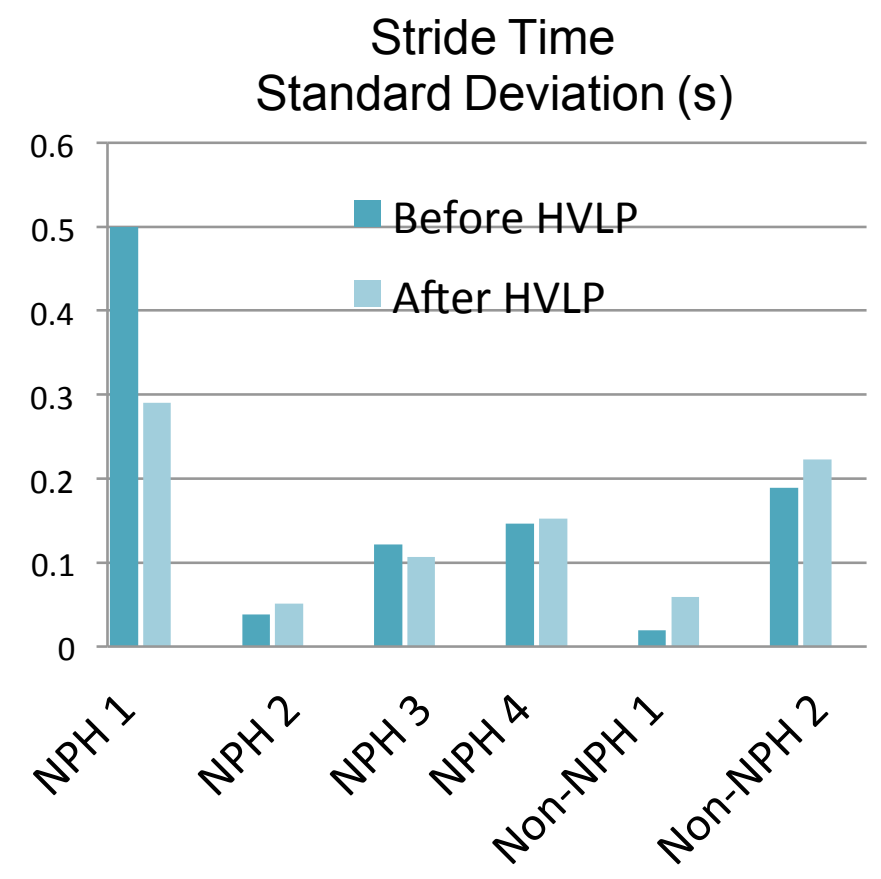

Figure 56 Average Stride Time Standard Deviation Change of Suspected NPH Patients Pre- to Post-HVLP Does Not Provide NPH Separability 
Higher stride time standard deviation indicates a higher temporal variability between strides, as suggested in [82]. However, the results shown in Figure 56 reveal that stride time standard deviation does not provide separability between the NPH and non-NPH patients (comparing intra-subject pre- to post-HVLP improvements).

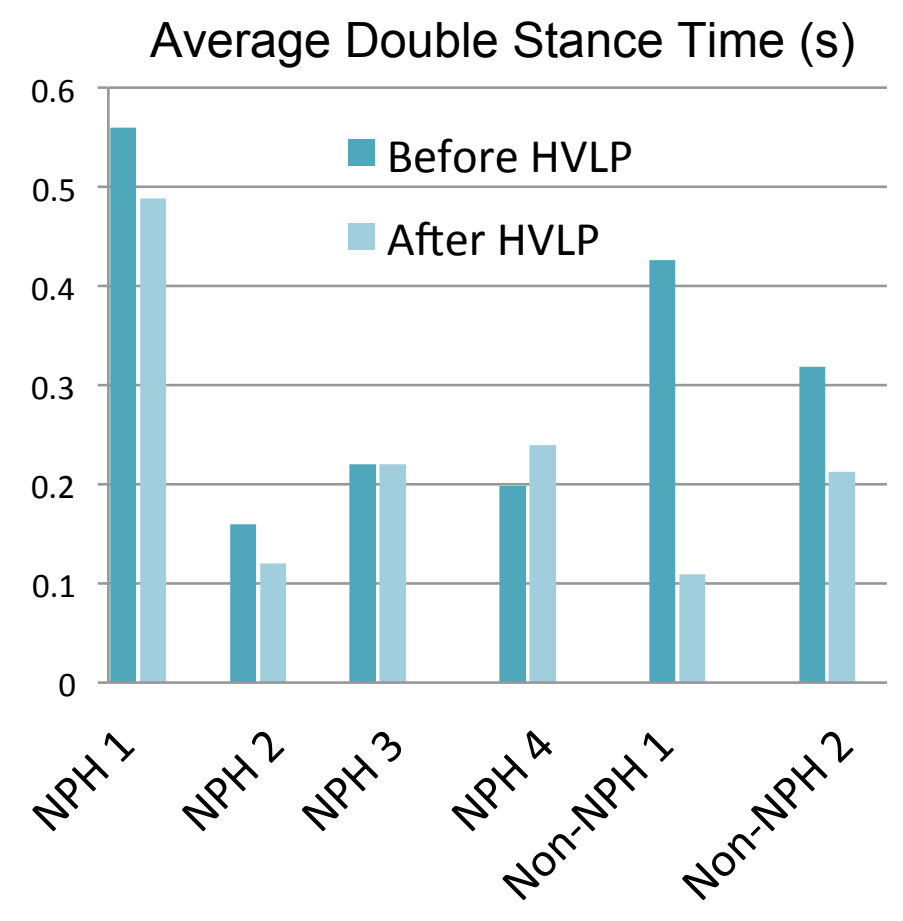

Figure 57 Average Double Stance Time Change of Suspected NPH Patients Pre- to Post-HVLP Does Not Provide NPH Separability

Higher average double stance time typically indicates issues with stability and balance, but the results in Figure 57 show that this feature does not always decrease after HVLP for the NPH patients and thus does not provide the desired separability for NPH diagnosis. 


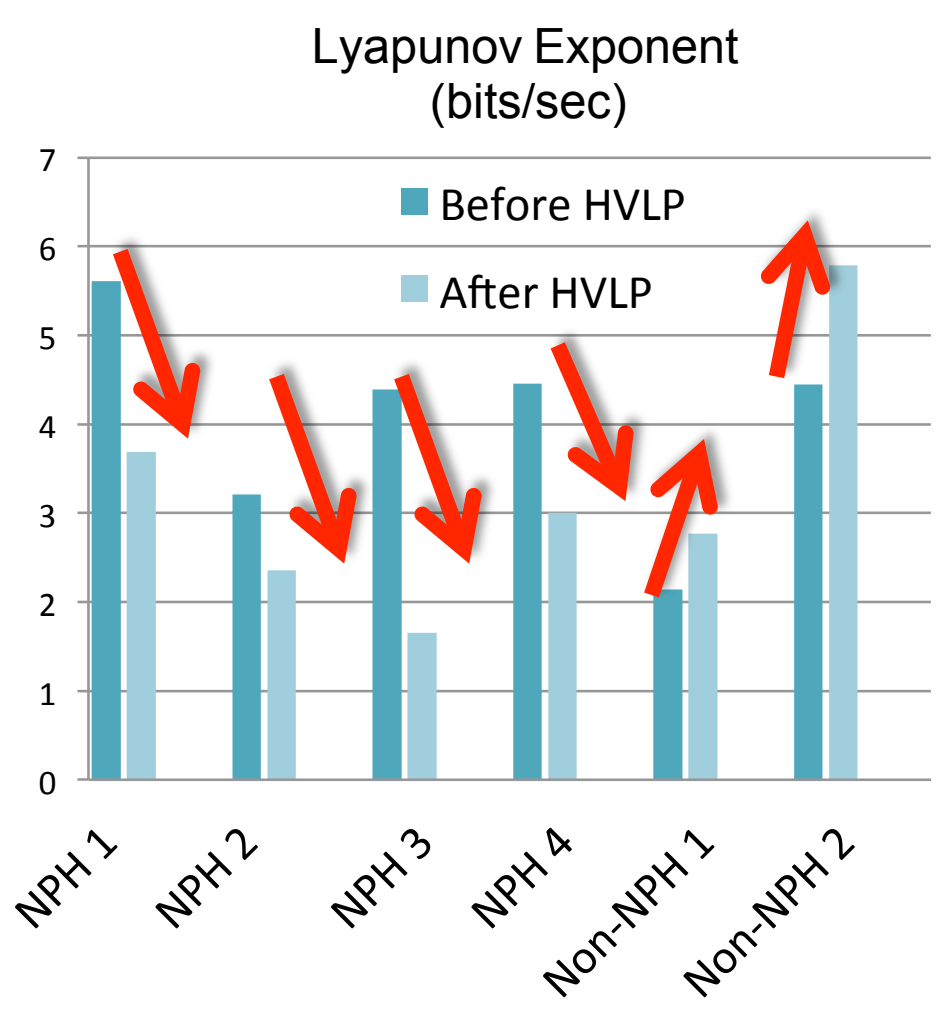

Figure 58 LyE Change of Suspected NPH Patients Pre- to Post-HVLP Does Provide NPH Separability (NPH patients improved post-HVLP (i.e., lower LyE), while nonNPH patients deteriorated), Indicating Its Potential to Aid in NPH Diagnosis

Figure 58 reveals that all diagnosed NPH patients see a significant post-HVLP improvement in stability (i.e., a decrease of LyE), and the non-NPH patients see no such improvement and even degradation (attributed clinically to fatigue). LyE therefore provides the desired NPH separability. To find statistical meaning in this preliminary finding, a two way ANOVA analysis was done on each gait feature extracted from above, and LyE was the only parameter that found before and after difference in the shunted group $(\mathrm{p}<0.025)$. This pilot data had an effect size greater than 1 , suggesting that a 
meaningful conclusion can be obtained with about 10 subjects in each group in future studies.

\subsubsection{Discussion}

As noted in Section 6.2.4, conventional observation-based gait parameters did not provide such separability. Though many researchers have found that gait speed and stride length are important parameters for diagnosis of NPH, the change in gait speed did not correlate well with the neurosurgeon's diagnoses. This could be due to two reasons. First, the 10-meter stopwatch method for assessing gait speed is not entirely accurate because of the reasons introduced in 6.1.2. Second, the patients may need to adjust gait speed to increase stability, and usually this adjustment is achieved by slowing down in the elderly, as argued in [54] and [87]. Variability assessment of stride time and stride length also do not correlate with diagnosis, as is corroborated in [54] and [87].

The results in from Figure 58 suggest that stability quantified by LyE is a good gait parameter for evaluating the HVLP response. Of all the parameters extracted, LyE provides the best separability for classifying those who are highly likely to have NPH and those who are not. It is again worth noting that this is clearly a small, underpowered pilot study, and this research merely provides promising pilot results within this context. However, the analysis is suggestive of the potential of using inertial BSNs for NPH diagnosis. Ultimately, a large-scale study is needed to further evaluate these and other BSN-enabled gait features for NPH diagnosis and other neuropsychological diseases. 


\subsection{Early Diagnosis of Multiple Sclerosis}

\subsubsection{Medical Background}

Multiple Sclerosis (MS) is estimated to affect 1/1000 individuals with an average age of onset between 20-40 years translating to 400,000 people affected in the United States. It is a chronic autoimmune disorder of the central nervous system (CNS) characterized by recurrent episodes of CNS inflammation and progressive neurodegeneration. As many other neuromuscular pathologies, collectively, the acute and chronic elements of MS disease activity result in accumulation of neurologic impairment and functional disability overtime. Walking performance is, therefore, an important outcome to assess severity of disease, disease progression, and therapeutic efficacy. More specifically, it is important to identify the gait parameters that are uniquely presented in MS subjects with objective and precise measurement and analysis.

\subsubsection{Hypothesis}

Current methods for assessing walking performance usually include a 6-minute walk, with a stopwatch and tape measure recording gait speed as a primary parameter. However, such method lacks of 1) high precision to capture various and subtle gait features, failing to record various gait impairment symptoms; 2) portability for longitudinal, continuous monitoring, failing to record gait degradation in natural environment and real life.

To better assess gait performance in MS for their diagnosis, we adopt more precise measurement tool with longitudinal monitoring capability - inertial BSN - to collect gait 
data of the MS group and healthy group. To determine whether the MS population and healthy population present significant difference in gait performance, various gait features will be extracted from the time series data collected by inertial sensors and evaluated by statistical methods.

\subsubsection{Experiment Setup}

28 study subjects (classified as mild MS, moderate MS and healthy control) wearing inertial sensors on the lower limbs were asked to undergo a fixed six minute walk (6MW) in a clinic. Followed by a medical assistant with a distance wheel recording the distance they walked during each minute, subjects were asked to walk as fast as possible (without running) up and down a hallway in the clinic, pivoting when they reached the end. The inertial sensor data was wireless transmitted to and recorded in a laptop placed in the hallway for post signal processing and analysis.

\subsubsection{Feature Extraction}

With the inertial data sampled at $128 \mathrm{~Hz}$, high precision gait features were extracted including: double stance time (DST), single support time (SST), swing time (SWT), gait cycle span (CYC) and ratio combinations of these four features (i.e. DST/CYC, SST/CYC, SWT/CYC, DST/SST, DST/SWT, and SWT/SST, techniques detailed in Chapter 3, Section 3.1.3), and gait complexity measured by elliptical Fourier analysis (techniques detailed in Chapter 4, Section 4.1.3.1). 


\subsubsection{Results}

These features were then evaluated by Cohen-D metric for their effect size (ES) between the MS group and healthy control group. Among the features extracted, the DST/CYC, $\mathrm{SST} / \mathrm{CYC}$, DST/SST, and SWT/SST have all presented an ES larger than 0.9, suggesting a large statistical and clinical difference between MS group and healthy control groups in these extracted gait features.

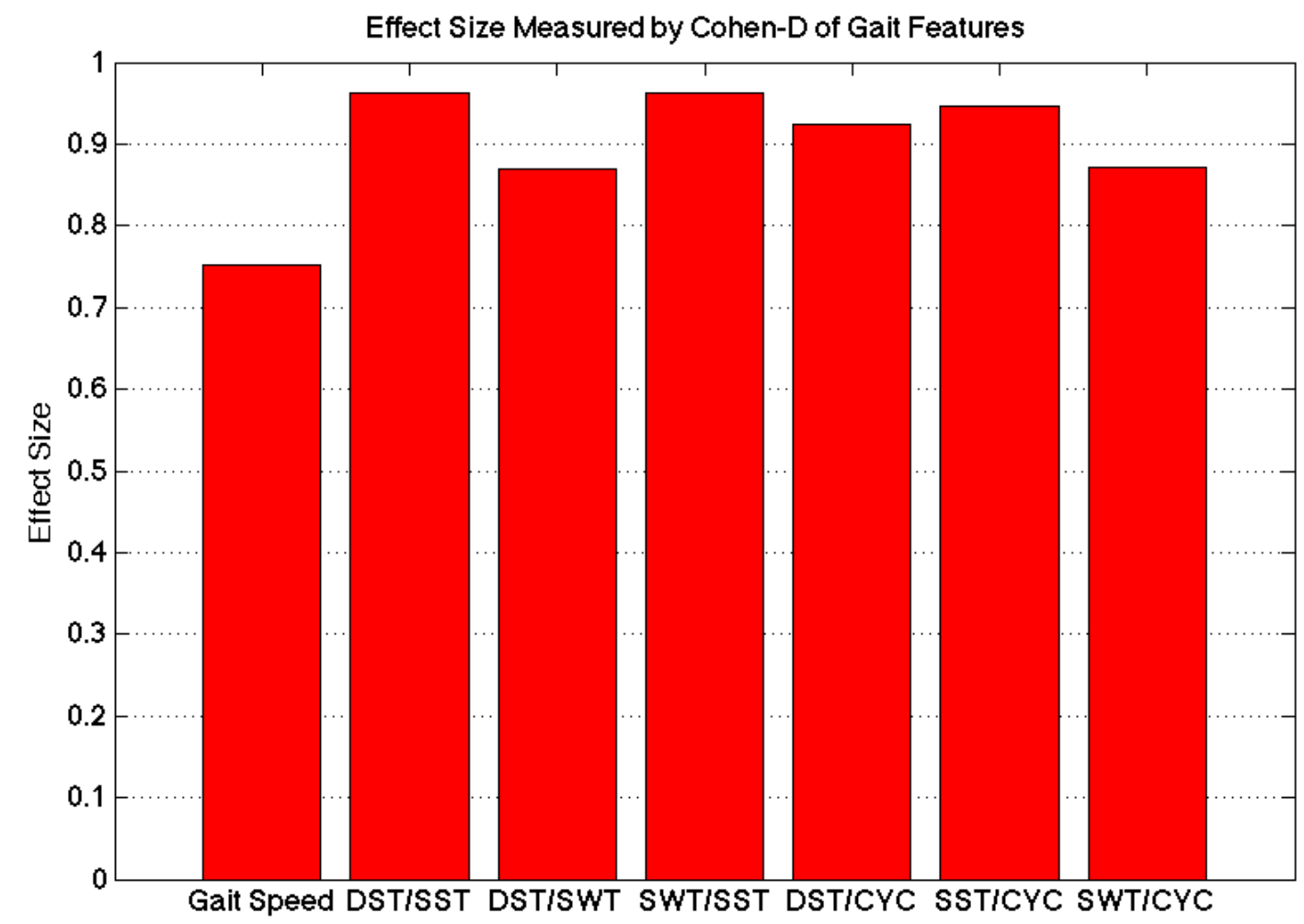

Figure 59 Effect Size Measured by Cohen's D of Some Gait Features

\subsubsection{Discussion}

We conducted experiments on MS subjects using wearable inertial sensors and extracted gait features from the inertial sensor data. These early results shed light on including gait 
analysis in MS assessment using wearable inertial sensors. These gait features, which is not available in current clinics, can bring tremendous amount of new information in the early diagnosis and drug intervention for the MS patients. Ongoing work will further examine how feature gait features will inform differential diagnosis in early MS.

\subsection{Summary}

This chapter integrated the techniques presented from Chapter 2 4 and applied them to the real-world medical applications. Through a series of case studies: assessing efficacy of ankle-foot orthoses for children with cerebral palsy, aiding diagnosis of normal pressure hydrocephalus, and enhancing gait assessment for early multiple sclerosis, this key chapter has demonstrated the feasibility of adopting inertial BSN for high precision, portable gait analysis. Gait features were identified and extracted from real patient data collected from medical pilot studies and clinic setting, with statistic analysis to showcase the competence of inertial BSNs for propel medical research that requires high precision gait analysis, and its promising prospect for longitudinal gait analysis in the natural environment. 


\section{Chapter 7}

\section{Conclusion and Future Work}

The emergence of Body Sensor Networks (BSNs) has sprouted the medical applications which most benefit from quantitative and objective measurement of human body. Inertial BSNs, which naturally captures the motion of human body, has demonstrated great potential for this purpose. With advanced techniques in first-principles modeling and machine learning, gait analysis using inertial BSNs has shown its own significance with multiple applications in medicine. The efforts in minimizing the form factor and power consumption of these sensors will further propel the prevalence of applying inertial BSNs for gait analysis in medical research and clinical practice.

\subsection{Final Summary}

This dissertation details methods of gait feature extraction from inertial BSNs targeting medical applications. With a broad horizon, this work depicts a systematical approach of retrieving information from inertial BSN data, as illustrated in Figure 60. Taking two possible paths - first-principles modeling and machine learning, the approach has 
extracted features ranging from the primary product such as kinematic information to more insightful ones educed from nonlinear analysis. With the knowledge of extracting various gait feature products, hypothesis of gait performance related medical applications were designed and tested via either machine learning approach or statistical analysis.

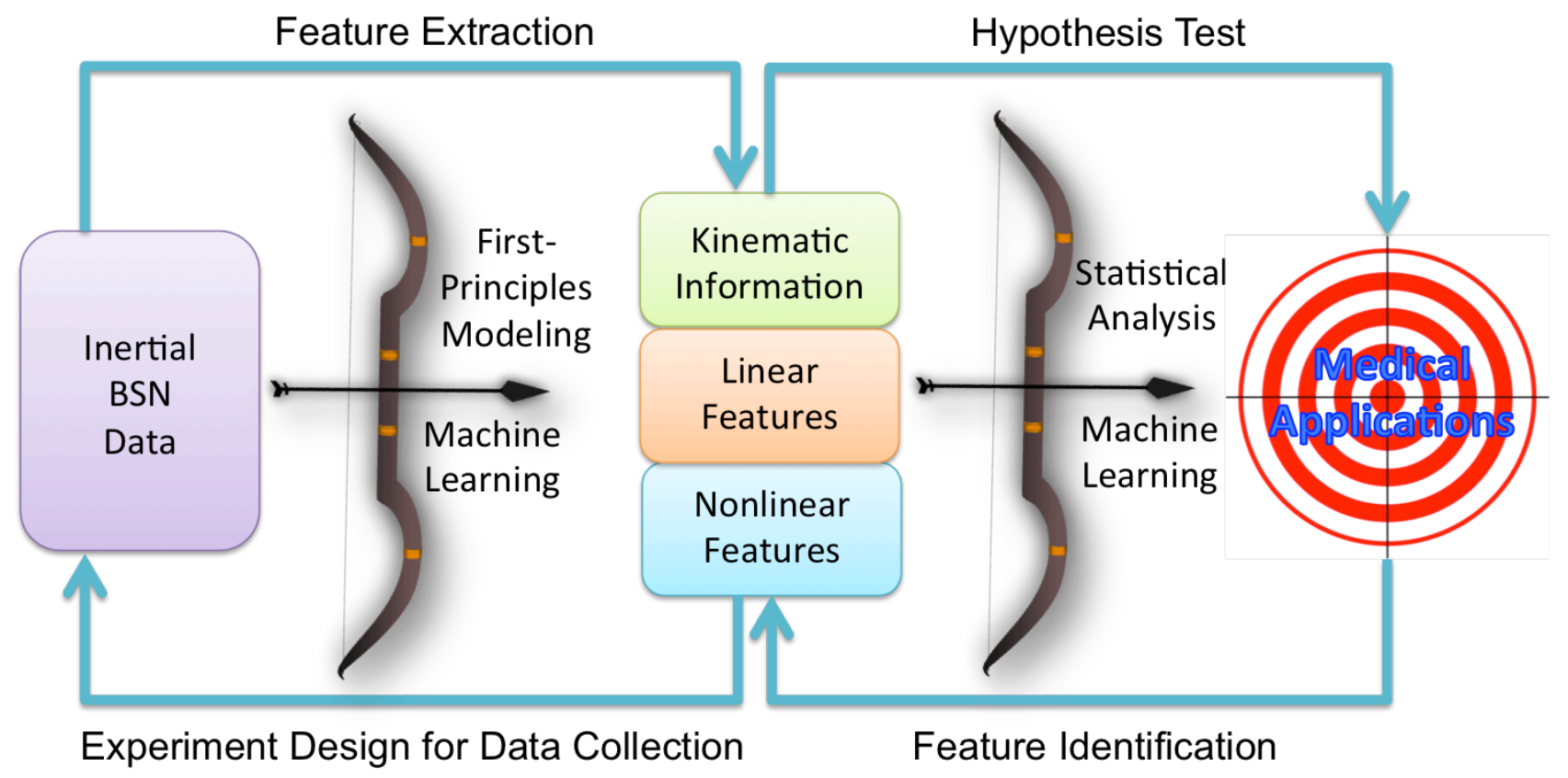

Figure 60 Final Summary of Dissertation

The road map shown in Figure 60 highlights the guideline of the methodologies running through Chapter 2 to Chapter 6. From the primary product - kinematic information from Chapter 2, to linear features and nonlinear features from Chapter 3 and Chapter 4, inertial BSN data have been cleaned and processed from preliminary physical quantities - noisy acceleration and angular velocity - to interpretable time series and quantitative measures, including motion range, joint angle, gait temporal features, and nonlinear features such as gait complexity and gait stability. With such interpretable information about human gait, 
statistical analysis can then be conducted for a particular medical application where gait assessment plays a key role. Furthermore, the choice of methodology is carefully studied by examining the advantage of first-principles modeling and machine learning. With a case example - extracting gait speed - this work has demonstrated how to combine the first-principles modeling and machine learning. Furthermore, this hybrid approach has also been tentatively applied to medical diagnosis that involves a wide range of gait characteristics such as multiple sclerosis.

\subsection{Future Work}

Future work includes extending the current in-lab method to the longitudinal, natural environment setting (Figure 61). Whilst the technologies hardware design advances with more flexible, easy to use, power efficient BSN platform coming up - novel signal processing techniques will be required to enable new applications. In future, techniques that further combine machine learning and first-principles modeling need to be explored. This is particularly important for further extending this research to longitudinal studies where the amount of data requires both first-principles knowledge to provide insight and machine learning techniques to provide standard automation procedure. 


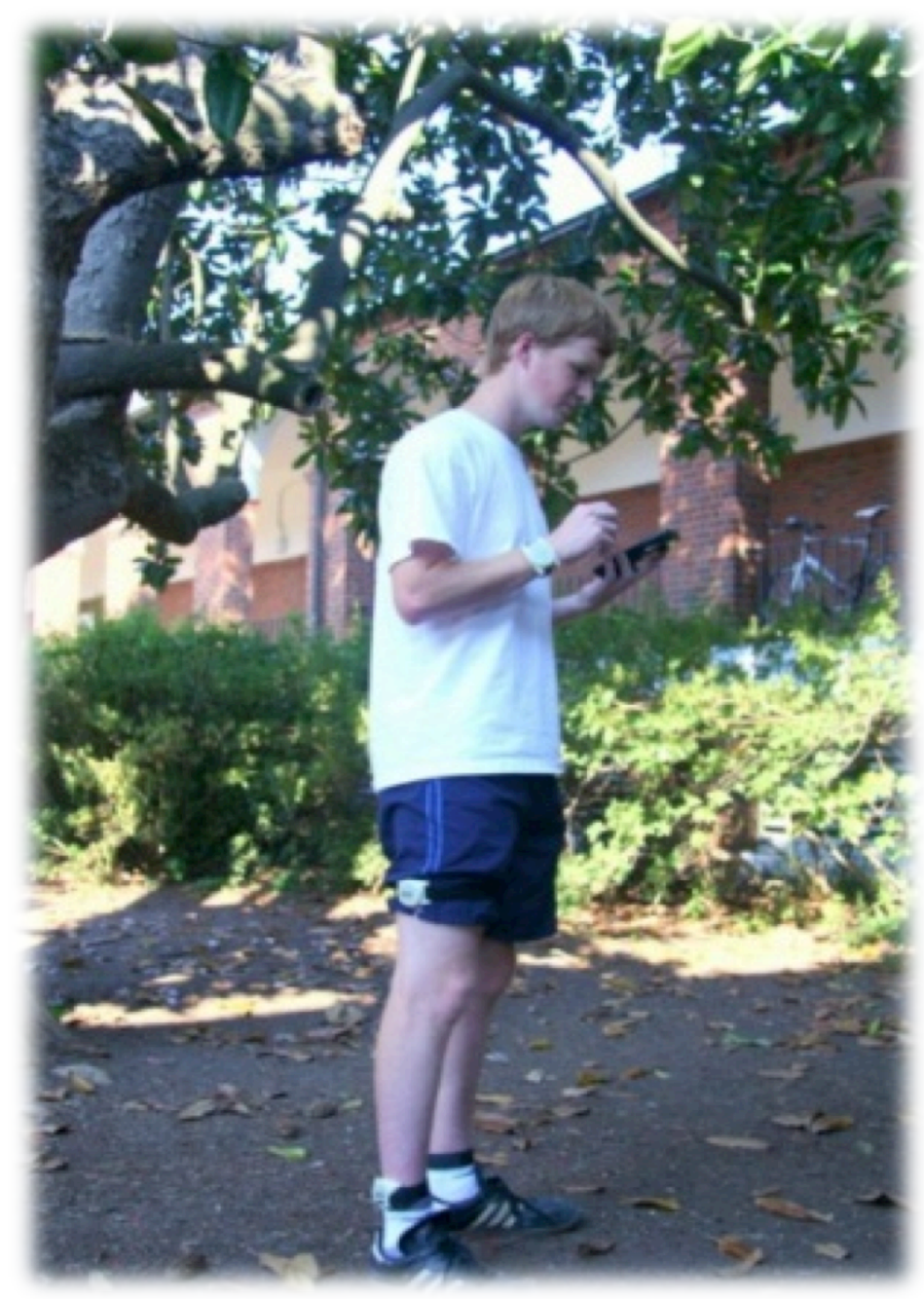

Figure 61 Inertial BSN Enabled Mobile Gait Analysis in Natural Environment

First, in the real-world longitudinal studies, gait features must be identified from the walking period automatically without dependence on user logging or annotation. Therefore, the real-world deployment usually requires intelligent activity identification. The time series of inertial sensor data are segmented into dynamic parts and static parts by their time sample indexes, by slicing the data stream, one could find the data set of 
interest. With machine learning techniques, better activity classification accuracy can be achieved for better pinpoint the activities and extracting relevant gait features from.

Second, adopting gait analysis as a standard procedure in clinics can have a significant impact for the diagnosis of symptoms where gait performance is a critical factor. For example, in the ongoing project of enhancing gait assessment of multiple sclerosis, including high precision gait analysis results obtained by deploying inertial BSNs can provide much more rich information than the current gait speed measurement - without the cumbersomeness of installing industrial standard optical motion capture system.

Third, this work can be extended to fall risk assessment via longitudinal monitoring. Falls can be detrimental to the elderly who are prone to them. Due to aging, the degradation in both sensory and ambulatory function cause instable gait and falls. Previous studies on falls are mostly limited to in-lab setting where falls are triggered by experimenters, subjecting the data to unnatural and unrealistic. With the capability of wearable sensors, it is possible to reexamine falls and monitor fall-related degradation of the elderly in natural environment, and alarm those whose gait become less stable and more prone to falls to take cautions, and finally prevent dysfunctions or mortality rate caused by falls. The next step is to deploy these wearable sensors to in-home and longitudinal monitoring for more meaningful experiments. 


\section{References}

[1] G. Z. Yang, "Body Sensor Networks", Springer-Verlag New York Inc, 2006.

[2] M. A. Hanson, H. C. Powell, A. T. Barth, K. Ringgenberg, B. H. Calhoun, J. H. Aylor, J. Lach, "Body area sensor networks: challenges and opportunities," Computer, vol. 42, no. 1, pp. 58-65, 2009.

[3] J. K. Oh, S. Cho, W. Bang, W. Chang, E. Choi, J. Yang, J. Cho, D. Y. Kim, "Inertial sensor based recognition of 3-D character gestures with an ensemble of classifiers", In Proceedings of the Ninth International Workshop on Frontiers in Handwriting Recognition (IWFHR '04), pp. 112-117, 2004.

[4] T. Kim, S. Chen, J. Lach, "Detecting and preventing forward head posture with wireless inertial body sensor networks," International Conference on Body Sensor Networks, 2011.

[5] L. Bao, S. S. Intille, "Activity recognition from user-annotated acceleration data", In Pervasive Computing”, Springer Berlin/Heidelberg, pp. 1-17, 2004.

[6] R. Jafari, W. Li, R. Bajcsy, S. Glaser, S. Sastry, "Physical activity monitoring for assisted living at home," International Conference on Body Sensor Networks, pp. 213-219, 2007.

[7] S. Chen, C. L. Cunningham, B. C. Bennett, J. Lach, "Enabling longitudinal assessment of ankle-foot orthosis efficacy for children with cerebral palsy", Wireless Health, 2011.

[8] Q. Li, J. A. Stankovic, M. A. Hanson, A. T. Barth, J. Lach, G. Zhou "Accurate, fast fall detection using gyroscopes and accelerometer-derived posture information”, Body Sensor Networks, pp. 138-143, 2009.

[9] A. Salarian, H. Russmann, F. J. G. Vingerhoets, C. Dehollaini, Y. Blanc, P. R. Burkhard, K. Aminian, "Gait assessment in Parkinson's disease: toward an 
ambulatory system for long-term monitoring", IEEE Transactions on Bio-Medical Engineering, vol. 51, no. 8, pp. 1434-1443, 2004.

[10] B. Noris, M. Nobile, L. Piccinini, M. Berti, E. Mani, M. Molteni, F. Keller, D. Campolo, A. G. Billard, "Gait analysis of autistic children with echo-state networks", Worshop on Echo State Networks and Liquid State Machines, 2006.

[11] A. Shrinivasan, M. Brandt-Pearce, A. T. Barth, and J. Lach, "Analysis of gait in patients with normal pressure hydrocephalus," International Workshop for Mobile Systems, Applications, and Services for Healthcare, pp.1-6, 2011.

[12] S. Chen, A. T. Barth, J. T. Barth, B. C. Bennett, M. Brandt-Pearce, D. K. Broshek, J. R. Freeman, H. L. Samples, and John Lach, "Aiding diagnosis of normal pressure hydrocephalus with enhanced gait feature separability", In Proceedings of the conference on Wireless Health (WH '12), 2012.

[13] A. T. Barth, B. C. Bennett, B. Boudaoud, J. S. Brantley, S. Chen, C. L. Cunningham, T. Kim, H. C. Powell, Jr., S. A. Ridenour, J. Lach, "Longitudinal high-fidelity gait analysis with wireless inertial body sensors," In Proceedings of the conference on Wireless Health (WH '10), pp. 192-193, 2010.

[14] A.T. Barth, M. A. Hanson, H. C. Powell, Jr., J. Lach, "TEMPO 3.1: A body area sensor network platform for continuous movement assessment," International Conference on Body Sensor Networks, pp. 71-76, 2009.

[15] I. Frosio, F. Pedersini, N.A. Borghese, "Autocalibration of MEMS accelerometers", IEEE Transactions on Instrumentation and Measurement, pp. 2034-2041, 2009.

[16] P. Lukowicz, H. Junker, G. Tröster, "Automatic calibration of body worn acceleration sensors", Pervasive Computing, pp. 176-181, 2004.

[17] J. Borenstein, L. Ojeda, S. Kwanmuang, "Heuristic reduction of gyro drift in IMU-based personnel tracking systems", SPIE Defense, Security + Sensing, Conference 7306A: Optics and Photonics in Global Homeland Security VI, $73061 \mathrm{H}, 2009$.

[18] A. Krohn, M. Beigl, C. Decker, U. Kochendörfer, P. Robinson, T. Zimmer. "Inexpensive and automatic calibration for acceleration sensors", 2005.

[19] D. Bannach, O. Amft, P. Lukowicz, "Automatic event-based synchronization of multimodal data streams from wearable and ambient sensors", European Conference on Smart Sensing and Context, pp. 135-148, 2009. 
[20] K. Arvind, "Probabilistic clock synchronization in distributed systems", IEEE Transactions on Parallel and Distributed Systems, vol. 5, no. 5, pp. 474-487, 1994.

[21] B. Sundararaman, U. Buy, A. D. Kshemkalyani, "Clock synchronization for wireless sensor networks: a survey”, Ad Hoc Networks, vol. 3, no. 3, pp. 281-323, 2005 .

[22] S. Chen, J. S. Brantley, T. Kim, J. Lach, "Characterizing and minimizing sources of errors in inertial body sensor networks," BodyNets, 2010.

[23] A. M. Sabatini, "Quaternion-based extended Kalman filter for determining orientation by inertial and magnetic sensing", IEEE Transactions on Biomedical Engineering, vol.53, no.7, pp.1346-1356, 2006.

[24] D. Roetenberg, P. J. Slycke, P. H. Veltink, "Ambulatory position and orientation tracking fusing magnetic and inertial sensing", IEEE Transactions on Biomedical Engineering, vol. 54, no.5, pp. 883 -890, 2007.

[25] J. L. Marins, X. Yun, E. R. Bachmann, R. B. Mcghee, M. J. Zyda, “An extended Kalman filter for quaternion-based orientation estimation using MARG sensors", IEEE/RSJ International Conference on Intelligent Robots and Systems,., vol. 4, pp. 2003-2011 , 2001.

[26] E. Shin, N. El-Sheimy, "An unscented Kalman filter for in-motion alignment of low-cost IMUs", Position Location and Navigation Symposium (PLANS), vol. 273, no.279, pp. 26-29, 2004.

[27] Z. Zhang, J. Pansiot, B. Lo, G. Yang, "Human back movement analysis using BSN", International Conference on Body Sensor Networks (BSN), vol. 13, no. 18, pp. 23-25, 2011.

[28] A. D. Young, "Wireless realtime motion tracking system using localised orientation estimation", PhD thesis, University of Edinburgh, 2010.

[29] C. Kirtley, "Clinical gait anlaysis: theory and practice", $1^{\text {st }}$ edition, 2006.

[30] M. W. Whittle, "Gait analysis: an introduction", $2^{\text {nd }}$ edition, 1996.

[31] R. L. Craik, C. S. Oatis, "Gait analysis: theory and application", $1^{\text {st }}$ edition, 1995.

[32] I. W. Griffiths, "Principles of biomechanics \& motion analysis", $1^{\text {st }}$ edition, 2005.

[33] G. Robertson, G. Caldwell, J. Hamill, G. Kamen, S. Whittlesey, "Research methods in biomechanics", $1^{\text {st }}$ edition, 2004. 
[34] D. A. Winter, "Biomechanics and motor control of human movement", $4^{\text {th }}$ edition, 2009.

[35] S. Studenski, S. Perera, K. Patel, C. Rosano, K. Faulkner, M. Inzitari, J. Brach, J. Chandler, P. Cawthon, E. B. Connor, M. Nevitt, M. Visser, S. Kritchevsky, S. Badinelli, T. Harris, A. B. Newman, J. Cauley, L. Ferrucci, J. Guralnik, "Gait speed and survival in older adults", Journal of the American Medical Association, vol. 305, no. 1, 2011.

[36] S. Miyazaki, "Long-term unrestrained measurement of stride length and walking velocity utilizing a piezoelectric gyroscope," IEEE Transactions on Biomedical Engineering, vol. 44, no. 8, pp. 753-759, 1997.

[37] S. Chen, C. L. Cunningham, B. C. Bennett, J. Lach, "Extracting spatio-temporal information from inertial body sensor networks for gait speed estimation," International Conference on Body Sensor Networks, 2011.

[38] A. M. Sabatini, C. Martelloni, S. Scapellato, F. Cavallo, "Assessment of walking features from foot inertial sensors", IEEE Transactions on Biomedical Engineering, vol. 52, no. 3, pp. 486-494, 2005.

[39] S. Yang. A. Laudanski, Q. Li, "Inertial sensor-based methods in walking speed estimation: a systematic review", Medical and Biological Engineering and Computing, vol. 50, no. 4, pp. 383-393, 2012.

[40] L. Laudanski, S. Yang, Q. Li, "A concurrent comparison of inertia sensor-based walking speed estimation methods", IEEE conference on Engineering of Medical and Biological Society, 2011.

[41] H. Vathsangam, B. A. Emken, D. Spruijt-Metz, G. S. Sukhatme, "Toward freeliving walking speed estimation using Gaussian process-based regression with onbody accelerometers and gyroscopes", 4th International Conference on Pervasive Computing Technologies for Healthcare (Pervasive Health), vol. 1, no. 8, pp. 2225,2010 .

[42] K. Nagarajan, N. Gans, R. Jarari, "Modeling human gait using a Kalman filter to measure walking distance", $2^{\text {nd }}$ Conference on Wireless Health (WH'11), 2011.

[43] E. Martin, "Novel method for stride length estimation with body area network accelerometers", IEEE Topical Conference on Biomedical Wireless Technologies, Networks, and Sensing Systems (BioWireleSS), vol. 79, no. 82, pp. 16-19, 2011.

[44] E. Martin, "Determination of a patient's speed and stride length minimizing hardware requirements", 2011 International Conference on Body Sensor Networks (BSN), pp. 23-25, 2011. 
[45] A. Panagiota, S. Layal, H. Stefan, "Assessment of human gait speed and energy expenditure using a single triaxial accelerometer", International Conference on Wearable and Implantable Body Sensor Networks (BSN), pp. 9-12, 2012.

[46] J. V. José, E. J. Saletan, “Classical dynamics: a contemporary approach”, 1998.

[47] C. J. Winstein, A. Garfinkel, "Qualitative dynamics of disordered human locomotion: a preliminary investigation", Journal of Motor Behavior, vol. 21, no. 4, pp. 373-391, 1989.

[48] J. E. Clark, S. J. Philips, "A longitudinal study of intralimb coordination in the first year of independent walking: a dynamic systems analysis", Child Development, vol. 64, no. 4, pp. 1143-157, 1993.

[49] Y. Hurmuzlu, C. Basdogan J. J. Carollo, "Presenting joint kinematics of human locomotion using phase plane portraits and Poincaré maps," Journal of Biomechanics, vol. 27, no. 12, pp. 1495-1499, 1994.

[50] N. Stergiou, "Innovative analyses of human movement: analytical tools for human movement research", Human Kinetics, $1^{\text {st }}$ Edition.

[51] L. A. DiBerardino III, J. D. Polk, K. S. Rosengren, J. B. Spencer-Smith, E. T. Hsiao-Wecksler, "Quantifying complexity and variability in phase portraits of gait," Clinical Biomechanics, vol. 25, no. 6, pp. 552-556, 2010.

[52] F. P. Kuhl ,C. R. Giardina, "Elliptic Fourier features of a closed contour," Comput Graph Image Process, vol. 18, no. 3, pp. 236-258, 1982.

[53] K. P. Granata, T. E. Lockhart, "Dynamic stability differences in fall-prone and healthy adults", Journal of Electromyography and Kinesiology, vol. 18, no. 2, pp. 172-178, Apr. 2008.

[54] J. B. Dingwell, H. G. Kang, "Differences between local and orbital dynamic stability during human walking," Journal of Biomechanical Engineering, vol. 129, no. 4, pp. 586-593, 2007.

[55] M. T. Rosenstein, J. J. Collins, C. J. De Luca, "A practical method for calculating largest Lyapunov exponents from small data sets", Physica D, vol. 65, pp.117134, 1993.

[56] A. Wolf, J. B. Swift, H. L. Swinney, J. A. Vastano, "Determining Lyapunov exponents from a time series", Physica D, vol.16, pp.285-317, 1985.

[57] F. Cignetti, L. M. Decker, N. Stergiou, "Sensitivity of the Wolf's and Rosenstein's algorithms to evaluate local dynamic stability from small gait data sets", Annals of Biomedical Engineering, vol. 40, no. 5, pp. 1122-1130, 2011. 
[58] K. Aminian, B. Najafi, C. Büla, P. F. Leyvraz, Ph. Robert, "Spatio-temporal parameters of gait measured by an ambulatory system using miniature gyroscopes," Journal of Biomechanics, vol. 35, no. 5, pp. 689-699, 2002.

[59] Q. Li, M. Young, V. Naing, J. M. Donelan, "Walking speed and slope estimation using shank mounted inertial measurement units", Journal of Biomechanics, vol. 43, no. 8, pp. 1640-1643, 2010.

[60] X. Xu, M. A. Batalin, W. J. Kaiser, B. Dobkin, "Robust hierarchical system for classification of complex human Mobility Characteristics in the Presence of Neurological Disorders", International Conference on Body Sensor Networks, pp. 65-70, 2011.

[61] I. Kononenko, E. Simec, E., M. Robnik-Sikonja, "Overcoming the myopia of inductive learning algorithms with RELIEFF", Applied Intelligence, vol. 7, no. 1, pp. 39-55, 1997.

[62] R. Tibshirani, "Regression shrinkage and selection via the lasso", Journal of the Royal Statistical Society, Series B, vol. 58, no. 1, pp. 267-288, 1996.

[63] G. H. John, P. Langley, "Estimating continuous distributions in bayesian classifiers", the Eleventh Conference on Uncertainty in Artificial Intelligence, 1995.

[64] L. Breiman, "Random forest," Machine Learning, vol. 45, no. 1, pp. 5-32, 2001.

[65] L. Breiman, "Stacked regression," Machine Learing, vol. 24, no.1, pp. 49-64, 1996.

[66] J. H. Friedman, "Multivariate adaptive regression splines," Annals of Statistics, vol. 19, no.1, pp. 1-67, 1991.

[67] N. Draper, and H. Smith, "Applied regression analysis," 2nd Edition, 1981, New York: John Wiley \& Sons, Inc.

[68] J. H. Friedman, J. Bentely, R.A. Finkel, "An algorithm for finding best matches in logarithmic expected time, ACM Transactions on Mathematical Software, vol.3, no. 3, pp. 209-226, 1977.

[69] R. Kohavi, "A study of cross-validation and bootstrap for accuracy estimation and model selection", International Joint Conference on Artificial Intelligence, pp. 1137-1143, 1995.

[70] D. N. Condie, C. B. Meadows, "Report of a consensus conference on the lower limb orthotic management of cerebral palsy", International Society of Prosthetics and Orthotics, 1995. 
[71] L. M. Knutson, D. E. Clark, "Orthotic devices for ambulation in children with cerebral-palsy and myelomeningocele”, Physical Therapy, vol. 71, pp. 947-960, 1991.

[72] K. Tong, M. H. Granat, "A practical gait analysis system using gyroscopes", Medical Engineering \& Physics, vol. 21, pp. 87-94, 1999.

[73] M. M. Powell, P. D. Silva, T. Grindeland, "Effects of two types of ankle-foot orthoses on the gait of children with spastic diplegia", Developmental Medicine \& Child Neurology, vol. 31, pp. 8-9, 1989.

[74] S. S. Thomas, J. M. Mazur, N. Wright, T. Supan, "Quantitative assessment of AFOs for children with cerebral palsy", Developmental Medicine \& Child Neurology, vol. 31, 1989.

[75] G. D. Rigamonti, M. A. Williams, "The diagnosis and treatment of idiopathic normal pressure hydrocephalus" Nature Clinical Practice Neurology, vol. 2, no. 7, pp. 375-381, 2006.

[76] N. Tanaka, S. Yamaguchi, H. Ishikawa, H. Ishii, K. Meguro, "Prevalence of possible idiopathic normal-pressure hydrocephalus in Japan: the Osaki-Tajiri project", Neuroepidemiology, vol. 32, no. 3, pp. 171-175, 2009.

[77] A. Shrinivasan, M. Brandt-Pearce, A. T. Barth, J. Lach "Analysis of gait in patients with normal pressure hydrocephalus", International Workshop for Mobile Systems, Applications, and Services for Healthcare, pp. 1-6, 2011.

[78] P. Bugalho, J. Guimares, "Gait disturbance in normal pressure hydrocephalus: A clinical study”, Parkinsonism and Related Disorders, pp. 434-437, 2007.

[79] S. Chen, C. L. Cunningham, B. C. Bennett, J. Lach, "Enabling longitudinal assessment of ankle-foot orthosis efficacy for children with cerebral palsy", Wireless Health, pp.1-10, 2011.

[80] L. Atallah, G. J. Jones, R. Ali, J. Leong, B. Lo, G-Z. Yang, “Observing recovery from knee-replacement surgery by using wearable sensors", International Conference on Body Sensor Networks, pp. 29-34, 2011.

[81] I. Tien, S. D. Glaser, R. Bajcsy, D. S. Goodin, M. J. Aminoff, "Results of using a wireless inertial measuring system to quantify gait motions in control subjects", IEEE Transactions on Information Technology in Biomedicine, vol. 14, no. 4, pp. 904-915, 2010.

[82] L. D. Ravdin, H. L. Katzen, A. E. Jackson, D. Tsakanikas, S. Assuras, N. R. Relkin, "Features of gait most responsive to tap test in normal pressure 
hydrocephalus", Clinical Neurology and Neurosurgery, vol. 110, no. 5, pp. 455461, 2008.

[83] M. A. Williams, G. Thomas, B. de Lateur, H. Imteyaz, J. G. Rose, W. S. Shore, S. Kharkar, D. Rigamonti, "Objective assessment of gait in normal-pressure hydrocephalus", American Journal of Physical Medicine and Rehabilitation, pp. 2-3, 2007.

[84] J. B. Dingwell, J. P. Cusumano, "Nonlinear time series analysis of normal and pathological human walking", Chaos: An Interdisciplinary Journal of Nonlinear Science, vol. 10, no. 4, pp. 848-886, 2000.

[85] S. M. Bruijn, D. J. J. Bregman, O. G. Meijer, P. J. Beek, J. H. van Dieën, "Estimating dynamic gait stability using data from non-aligned inertial sensors", Annals of Biomedical Engineering, vol. 38, no. 8, pp. 2588-2593, 2010.

[86] H. G. Kang, J. B. Dingwell, "Dynamic stability of superior vs. inferior segments during walking in young and older adults", Gait and Posture, vol. 30, no. 2, pp. 260-263, 2009.

[87] S. A. England, K. P. Granata, "The influence of gait speed on local dynamic stability of walking", Gait and Posture, vol. 25, no. 2, pp. 172-178, 2007. 


\section{List of Publications}

[P1] S. Chen, J. Gong, J. Lach, M. D. Goldman, "Enhanced Multiple Sclerosis Gait Assessment using Inertial Sensors", Wireless Health, 2013;

[P2] S. Chen, J. Lach, "Nonlinear Feature for Gait Speed Estimation using Inertial Body Sensors", International Conference on Body Area Networks (BodyNets), 2013;

[P3] S. Chen, J. Lach, O. Amft, M. Altini, J. Penders, "Unsupervised Activity Clustering to Estimate Energy Expenditure with a Single Body Sensor", International Conference on Body Sensor Networks (BSN), 2013;

[P4] Q. Li, S. Chen, J. Stankovic, "Multi-Modal In-Person Interaction Monitoring Using Smartphone and On-Body Sensors", International Conference on Body Sensor Networks (BSN), 2013;

[P5] C. M. Archer, J. Lach, S. Chen, M. F. Abel, B. C. Bennett, "Activity Classification in Users of Ankle Foot Orthoses", Journal of Gait and Posture, 2013;

[P6] S. Chen, J. S. Brantley, T. Kim, S. A. Ridenour, J. Lach, "Characterising and Minimising Sources of Error in Inertial Body Sensor Networks", International Journal of Autonomous and Adaptive Communications Systems, 2012;

[P7] S. Chen, A. T. Barth, J. T. Barth, B. C. Bennett, M. Brandt-Pearce, D. K. Broshek, J.R. Freeman, H.L. Samples, J. Lach,"Aiding Diagnosis of Normal Pressure Hydrocephalus with Enhanced Gait Feature Separability", Wireless Health, 2012;

[P8] S. Chen, C. L. Cunningham, B. C. Bennett, J. Lach, "Enabling Longitudinal Assessment of Ankle-Foot Orthosis Efficacy for Children with Cerebral Palsy", Wireless Health, Best Paper Award, 2011; 
[P9] S. Chen, C. L. Cunningham, B. C. Bennett, J. Lach, "Extracting Spatio-Temporal Information from Inertial Body Sensor Networks for Gait Speed Estimation", International Conference on Body Sensor Networks, (BSN), 2011;

[P10] T. Kim, S. Chen, J. Lach, "Detecting and Preventing Forward Head Posture with Wireless Inertial Body Sensor Networks", International Conference on Body Sensor Networks, (BSN), 2011;

[P11] A. T. Barth, B. C. Bennett, B. Boudaoud, J. S. Brantley, S. Chen, C. L. Cunningham, T. Kim, H. C. Powell, Jr., S. A. Ridenour, J. Lach, "Longitudinal High-Fidelity Gait Analysis with Wireless Inertial Body Sensors", Wireless Health, 2010;

[P12] S. Chen, J. S. Brantley, T. Kim, J. Lach, "Characterizing and Minimizing Sources of Errors in Inertial Body Sensor Networks", International Conference on Body Area Networks (BodyNets), 2010;

[P13] S. Chen, S. Cheng, P. Verma, R. Huck, "Heuristic Algorithms for Designing Minimum Cost FSO Networks", IEEE 3rd International Symposium on Advanced Networks and Telecommunication Systems (ANTS), 2009;

[P14] P. Verma, A. Ghosh, R. Huck, S. Cheng, S. Chen, M. Martens, A. Kaul, "Designing a Wireless Sensor System for Continuous Monitoring of the Cervical Dilation of a Pregnant Woman", International Journal of Advanced Media and Communication, 2009;

[P15] P. Verma, A. Ghosh, R. Huck, S. Cheng, S. Chen, M. Martens, A. Kaul, "Continuous Wireless Monitoring of the Cervical Dilation of a Pregnant Women", IEEE International Workshop on Medical Measurement and Applications (MeMeA), 2009. 